\title{
Distributed absorber for noise and vibration control
}

\author{
Michel Azoulay ${ }^{\mathrm{a}, *}$, Alexander Veprik ${ }^{\mathrm{b}}$, Vladimir Babitsky ${ }^{\mathrm{a}}$ and Neil Halliwell ${ }^{\mathrm{a}}$ \\ ${ }^{a}$ Loughborough University, Loughborough, Leicestershire, LE11 $3 T U$ \\ ${ }^{\mathrm{b}}$ RICOR, Cryogenic \& Vacuum Systems, En Harod Ihud, 18960, Israel
}

Received 1 January 2010

Revised 1 August 2010

\begin{abstract}
An approach to a wide-band frequency passive vibration attenuation is introduced in this paper. This aims to suppress noise and vibration of extended multimode objects like plates, panels and shells. The absorber is arranged in the form of a single-layer assembly of small inertial bodies (balls) being distributed and moulded within the light visco-elastic media (e.g. silicone resin). The absorber as a whole is embedded into object face covering the critical patches of the system surface. For the purpose of characterization, the authors introduced the complex frequency response function relating the volume velocity produced by the vibrating object surface (response) stimulated by a point-wise force (stimulus) applied to a particular point. The simulation and optimization of the main frequency characteristics has been performed using a full scale 3-dimensional Finite Element model. These revealed some new dynamic features of absorber's structures, which can contribute to vibration attenuation. A full-scale physical experimentation with synthesised absorber's structures confirmed the main results of simulation and has shown significant noise reduction over a staggering $0-20 \mathrm{kHz}$ frequency band. This was achieved with a negligible weight and volume penalty due to the addition of the absorber. The results can find multiple applications in noise and vibration control of different structures. Some examples of such applications are presented.
\end{abstract}

Keywords: Noise and vibration control, passive vibration attenuation, wide frequency band absorber

\section{Introduction}

The advantage of having a wideband and global vibration attenuation device, with a low volume penalty pay may be beneficial in wide range of industrial applications. From daily home appliances to aerospace industries, the demand of having a more silent and hi-performance mechanical design is increasing. Currently, the most accepted way to tackle wide range structural born vibrations is by passive and active methods. These methods provide limited performance and in majority of cases involve high volume and weight penalty pay as well as cluttering the already packed mechanical design. The challenge of having a conceptual method that provides wide range vibration attenuation is yet to be fully explored. This work will show in detail the process of developing a specially designed mechanical structure that has numerous resonances over a wide frequency range and therefore, provides wideband vibration attenuation. This structure dynamics is based on an expansion upon the concept of the classical Tuned Dynamic Absorber (TDA) theory [1] and wave pressure phenomenon [2].

The most accepted passive methods of vibration attenuation are based on the theory of the tuneable dynamic absorber (TDA). Traditionally, a single TDA was used to reduce the displacement amplitude, caused by a forced harmonic vibration, at a specific region along the structure. Fundamentally, TDA may provide narrow frequency band vibration attenuation to a fairly small region of a continuous vibrating structure.

\footnotetext{
*Corresponding author. E-mail: michelazoulay@gmail.com.
} 


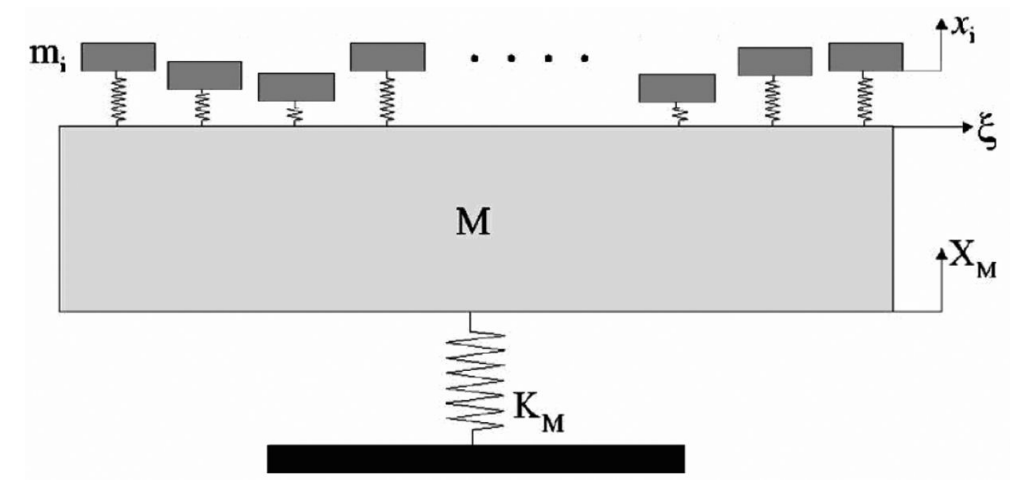

Fig. 1. A Schematic description of a primary deformable structure $(M)$ and attached set of Oscillators $\left(\mathrm{m}_{i}\right)$ [7].

The attachment of TDA to machinery and structures has been considered by many researchers in the past three quarters of the century. The first recorded use of a dynamic vibration absorber was described by Watts (1883) and followed by Frahm (1909), but it was not until 1928 that Ormondroyd and Den Hartog published the mathematical treatment of the passive dynamic vibration absorber. This section will review major approaches attempted to attenuate wideband vibration of a continuous system.

\subsection{Wideband vibration attenuation using TDA}

Expanding the limited capability of the TDA to attenuate the structural borne noise radiation of a continuous system was attempted by Brennan and Dayou [4]. A general mathematical model has been developed for a structure with many vibration neutralizers attached. In order to gain a physical insight into the passive control mechanisms, a beam with a single device attached has been studied. It was found that for local vibration control, where the vibration absorbers aim is to pin the structure at the point of attachment, the dynamic characteristics of the absorber are required to be tuned to a specific frequency. However, with global control the required dynamic characteristics of the neutralizer have optimum (threshold) values, beyond which any increase does not result in improved performance. The expression for this optimum values has been developed. However, adding a TDA may result in an increase rather than decrease in global vibration at some frequencies. These frequencies are the natural frequencies of the host structure when it is pinned at the neutralizer attachment point. If these natural frequencies coincide with a frequency of interest, they can be shifted to other frequencies by changing the position of the neutralizer (also emphasized by [5]). Simulation results show the kinetic energy of the cantilever beam calculated at each frequency with two control strategies implemented. It can be seen from the results that the constrained optimal control strategy reduces the kinetic energy at most frequencies and never makes the situation worse. The best control is achieved around the original resonance frequencies of the beam and there are some other frequency zones that are impossible to control. It is also clear that although the single TDA is effective at some frequencies, it makes global vibration worse at other frequencies.

\section{2. "Energy Sink" method}

A single TDA being a single degree of freedom (DOF) has little control over the overall structure. Using several TDA could broaden the effect on attenuation both by spectrally and spatially. Koc et al.1 [6,7] used an alternative method of vibration absorption referred to as 'energy sinks'. This method is based upon the attachment of a set of un-damped oscillators to a vibrating structure. According to the authors, these oscillators function as an energy sink which 'absorbs' kinetic energy from a structure and retains it in their phase space. However, in principle, these so called 'energy sinks' do not transform the kinetic energy to heat as in the classical sense. The absorbed energy remains at the 'energy sinks' permanently (or for a sufficiently long duration) so that the flow of energy from the primary structure appears as damped. The use of the term 'energy sinks' to name this method is not quite due to the fact that the total energy of the mechanical system remains unchanged and the sinking mechanism (in the sense of 


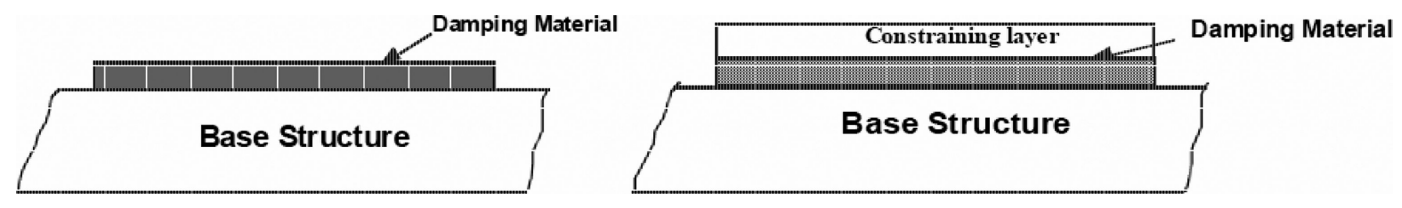

Fig. 2. Surface treatments: Free layer (left), Constraint layer (right) [9].

dissipation) has to be added, which can diminish an absorbing effect. The development of an optimization process was demonstrated to calculate the stiffness and mass properties of equally spaced oscillators along the structure (see Fig. 1).

The optimization process aims to minimize the energy of the primary mass over a selected time period. Optimization results show a degree of robustness of the process with respect to the initial frequency distributions and the values of loss factors. Optimum distributions of attached oscillators increase the density near the frequencies of interest. This result is consistent with the observation that with linear frequency distributions (and thus constant frequency difference) oscillators near the primary frequency respond with a higher level of energy absorption than those with frequencies away from it. Such a distribution also de-emphasizes the need to fine tune the frequencies as in conventional TDA. However, implementing the 'energy sink' method to a real structure demands high volume penalty due to the fairly large volume of oscillators needed to compensate for their lack of traditional viscous energy dissipation (that results in low loss factor). In practice, 'energy sinks' were found more suitable for large structures, and it has an advantage in transient and low frequency applications.

\subsection{Multi degree of freedom TDA}

An additional approach intended to broaden the effect of single degree of freedom TDA, is using a distributed parameter absorber which is comprised of multi DOF. Arpaci [8] used an annular plate to suppress the vibrations of continuous structures such as beams and plates. A full description is described by Arpaci and optimum values of its tuning and damping parameters are specified in a graphical form. The tuning aims are to achieve suppression of the first resonance, which is the most troublesome for structures. The main and auxiliary systems are assumed to have structural damping. With appropriate selection of the tuning and damping parameters, the fundamental resonance of the main system is separated into two new ones with considerably reduced amplitudes (as for the case of 1D TDA). The effectiveness of this approach results from the several structural resonances of the annular plate (making it function similar to several TDA's with different resonance frequencies connected to the same point along the structure). The vibration levels are attenuated both by the structural damping of the annular plate and its TDA effect. It was further suggested that relatively large damping factors can be obtained by coating the annular plate surface with damping compound or by using plates from several steel-viscoelastic laminations. This approach, referred to as 'Surface damping treatment', is discussed in the next section.

\subsection{Surface damping treatment}

One of the recent trends in passive damping technologies of mechanical structures is the use of various combinations of viscoelastic materials and metal skins. These techniques, referred to as 'surface damping treatments', are based on knowledge available since the 1950's (Kerwin 1959). Surface damping has renewed interest lately and is widely used to reduce interior noise and vibration in passenger vehicle structures. Two of the most common surface damping treatments, which are discussed in Rao 2003, are 'free layer damping' and 'constrained layer damping' (see Fig. 2). Free layer damping is accomplished when a damping material is either sprayed on the structure or bonded using a pressure-sensitive adhesive. When the base structure is deflected in bending, the viscoelastic material deforms primarily in tension and compression in planes parallel to the base structure. The hysteretic loop of the cyclic stress and strain dissipates the energy. The degree of damping is limited by thickness and weight restrictions.

Constraint layer surface treatment consists of the sandwich of two outer elastic layers with a viscoelastic material as the core. When the base structure undergoes bending vibration, the viscoelastic material is forced to deform in shear by the upper stiff layer. According to the authors, the constrained-layer damping is more effective than the 
free-layer design since more energy is consumed and dissipated into heat in the work done by the shearing mode within the viscoelastic layer. Marcotte et al. [10] conducted constraint layer surface treatment on a clamped beam and clamped plate structures. Different mass distributions were also appended by alternating the constraint layer thickness, in order to enhance the vibration attenuation of the surface treatment. The mass and layer distribution was optimized to minimize the mean square velocity field of the structure (equivalent to volume velocity). The results show a wideband vibration attenuation average of $13 \mathrm{~dB}$.

\subsection{Self damping system}

An original approach of a self damping system was introduced by Babitsky and Veprik [2]. This novel system which consists of an elastic beam with a sliding washer was found to 'self damp' under various external excitations. The sliding washer is found to eliminate the beam resonances by 'moving' along the beam. The movement of the washer results from the action of vibration induced force referred to as 'wave pressure' force. The self damping resulted from the detuning of the system 'string pulse washer' away from the original resonances consequently changing the system eigenfrequency spectrum. The dynamics of this system was formulated and also substantiated experimentally. Although this approach evoked greater interest, attributable to its enormous potential and applicability, it was only investigated again in a few publication ((Thomsen 1996) and (Miranda, Thomsen 1998)).

\subsection{Distributed dynamic absorber - A three dimensional self damping system}

This chapter reviews the various passive vibration control methods which attempt to reduce vibration in a continuous structure under wideband excitation. The classic TDA proved to be affective in a local reduction of structural vibration but insufficient when it comes to wideband vibration attenuation. Expanding upon the concept of TDA was attempted by introducing the multi degree of freedom TDA providing wideband attenuation. However, it proved to control only a limited space in the vicinity of its connection. The 'energy sinks' method seemed more appropriate for global and wideband vibration attenuation in terms of robustness with respect to the initial frequency distributions and the value of loss factors. Nevertheless, implementing the 'energy sink' method to a real structure demands high volume penalty pay.

The distributed dynamic absorber (DDA) presented below, intended to use the advantages of the aforementioned methods without paying a high volume penalty. It intends to provide wideband vibration attenuation regardless of the structure it is attached to. This may be achieved by expanding the mode of self damping system to a 3D system and the use of large ball bearing particles embedded in a viscoelastic matrix.

A schematic illustration of the proposed DDA is presented in Fig. 3. The viscous matrix is presented through a spring damper couple, and the ball bearing particles are presented with gray circles.

Nevertheless, in order to achieve a true wideband and large scale vibration attenuation the distributed dynamic absorber is introduced. The distributed dynamic absorber utilizes the advantages of several techniques of passive damping methods without paying a high mass or volume penalty. It is intended to provide wideband vibration attenuation regardless of the structure it is attached to and the excitation severances.

\section{Multi modal dynamic absorber}

\subsection{One dimensional dynamic absorber vs. three dimensional dynamic absorber}

TDA's are widely used in mechanical systems as a local vibration attenuation device. At the present, the majority of applications that use a classical TDA, use it as a 'pure' one degree of freedom (1D) device. In spite of the fact that a 1D TDA is essentially a three dimensional entity, it is designed in such manner that its two additional lateral resonances are significantly higher than its axial resonance, where the axial direction coincides with the excitation direction (see Fig. 4). This design mind-set originated out of the simplification of tuning an added 1D entity TDA to an existing mechanical system. In certain cases, where a three directional vibration absorption is required a threesome of TDA's are used. 

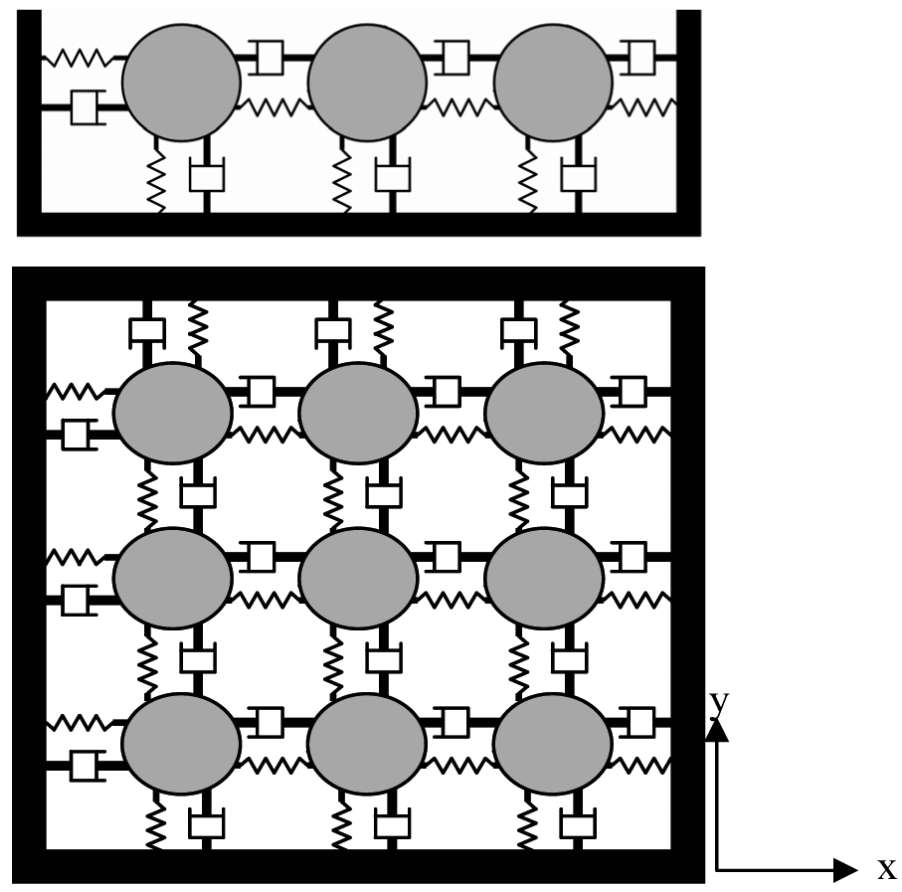

Fig. 3. Schematic model of DDA - Side view (upper sketch), Top view (lower sketch).

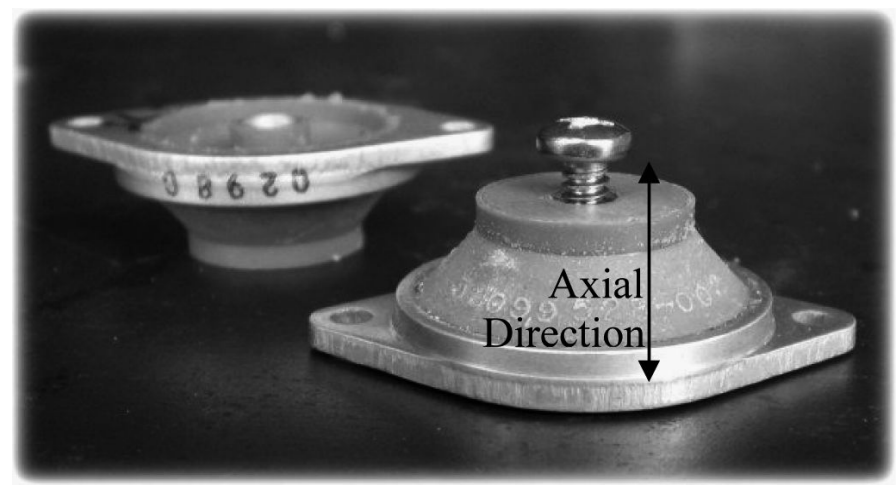

Fig. 4. Typical vibration isolator often used in commercial 1D TDA systems.

The above simplification results in missing out the key benefit of having additional modes for the TDA. The effectiveness of having some additional modes in direction other than the excitation direction is yet to be thoroughly explored and wrongfully considered as minor, by a rule of thumb. The next section will show the added value of the additional modes.

\subsubsection{A 3D dynamic absorber model}

A 3D Tuned Dynamic Absorber is a three dimensional mechanical entity rather than a one dimensional such as the classical 1D TDA. In order to emphasize the difference between a 3D TDA and a 1D TDA, a Finite Element (FE) model of a simple mass-spring-damper structure is used. Figure 5 illustrates the FE model which consists of a metal ball embedded in a viscoelastic matrix. Using this basic TDA structure enables straightforward tuning of its dynamic properties simply by either altering the visco-elastic matrix stiffness and damping, or varying its metal ball density. This structure concept has many other advantages which will be explained later in greater details. 


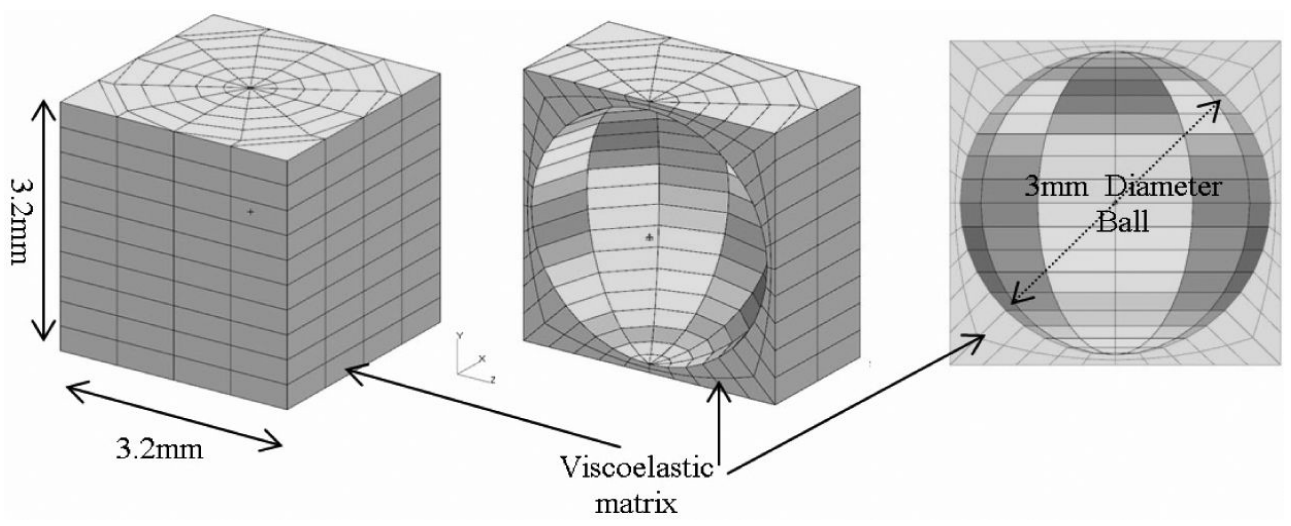

Fig. 5. FE model of a 3D mass-spring-damper structure.

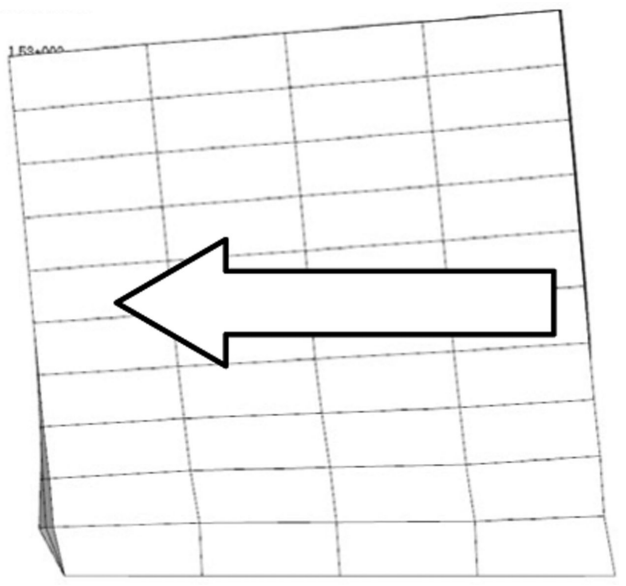

Fig. 6. TDA 1st and 2nd mode: $641 \mathrm{~Hz}-\mathrm{X}$ and $\mathrm{Z}$ translation (lateral).

\subsubsection{Normal modes of the 3D tuned dynamic absorber}

The first stage in understanding the dynamic behaviour of the 3D TDA is looking at its normal modes. Using a FE normal modes simulation, the first six modes generated are shown in Figs 6 to 9. The boundary condition used at this simulation is a constrained bottom face of the 3D TDA (the lower face that is on the X-Z plane see Fig. 5).

As seen in Figs 6 to 9, the 3D TDA has other five modes in addition to the typical 'axial' mode of the 1D TDA. These five modes have a distinctive dynamic behaviour totally different from a classical 1D mass-spring-damper system. Furthermore, since the aforementioned 3D TDA model represents a continuous structure, many more modes are generated in the FE simulation. Some of these modes are higher harmonics of the six first modes and some are a permutation of them. It is appropriate to mention here that certain modes are triggered in a certain direction of excitation, whereas other modes would be triggered in different excitation direction. In order to distinguish between the modes and to emphasize how does a 3D TDA model differs from the classical one, a frequency response curve is generated. The next section will show the profound added value the 3D TDA has over the classic 1D TDA.

\subsubsection{Frequency response curves of a $3 D$ and $1 D T D A$}

This section will show a comparison between two cases, one is the case of a classical mass-spring-damper namely a 1D TDA system and the other would be a 3D TDA. Both cases has been tuned to a $90 \mathrm{~Hz}$ resonance in the axial direction, have a mass of $10 \mathrm{~g}$ and damping with a loss factor of 0.2 . In fact, these two mechanical systems are fundamentally identical in a classical dynamics point of view. Both systems are subjected axially to a sine sweep procedure and the two frequency response curves generated are shown in the Fig. 10. 


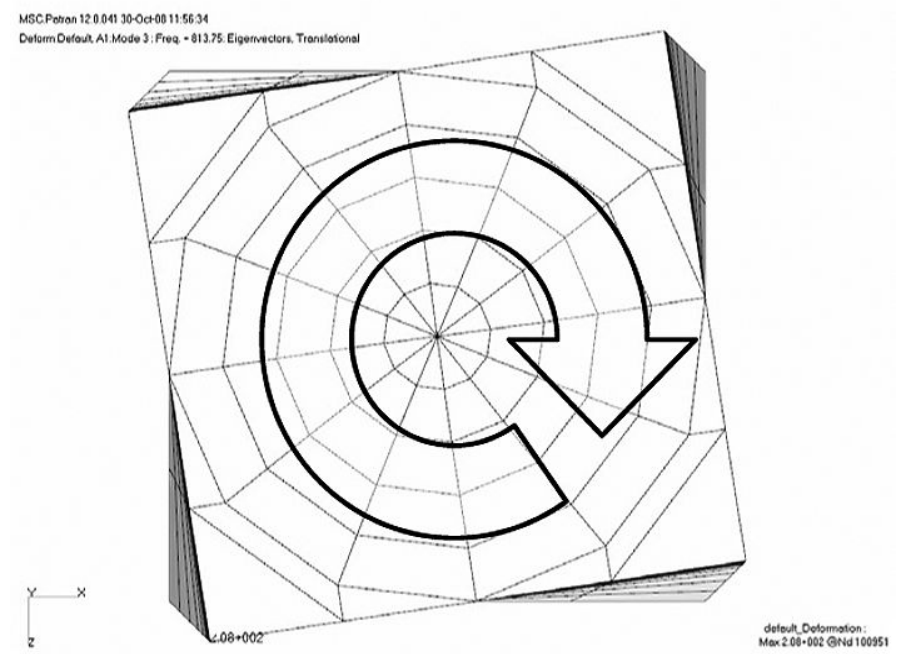

Fig. 7. TDA 3rd mode: $813 \mathrm{~Hz}-$ Y Rotation.

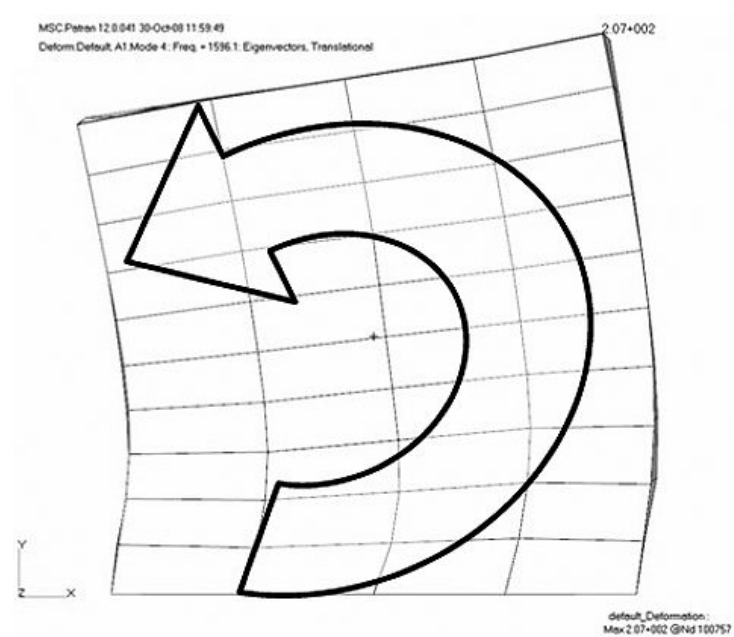

Fig. 8. TDA 4th-5th mode: $1596 \mathrm{~Hz}-\mathrm{X}, \mathrm{Z}$ Rotation.

It is evident from Fig. 10 that the frequency response of the 3D TDA is much more impressive than the 1D TDA. At the lower frequency band, both response curves seem the same, as the resonance of the 1D TDA and the 6th mode (axial) of the 3D TDA were tuned to $90 \mathrm{~Hz}$. On the other hand, at the above $200 \mathrm{~Hz}$ frequency range, it can be seen that the 3D TDA has many more resonances than the 1D TDA. This implies that the additional aforementioned modes uncovered here contribute to the 3D TDA dynamic absorption.

Having these additional modes, insinuate the existence of a dynamic absorption capacity, in a wider frequency range. Harnessing the multi modal dynamic absorption potential of the 3D TDA may bring us closer to the wide range vibration attenuation we seek.

The next stage would be testing the 3D TDA in a simple dynamic system to see the fruition of its wide spectrum absorption capacity.

\subsection{Velocity field, volume velocity and optimization criteria}

It is a known fact that the sound radiating from a vibrating structure is defined primarily by a normal component of its enclosure surface velocity. Let the velocity field along a mechanical system domain $(\Omega)$ defined by: 


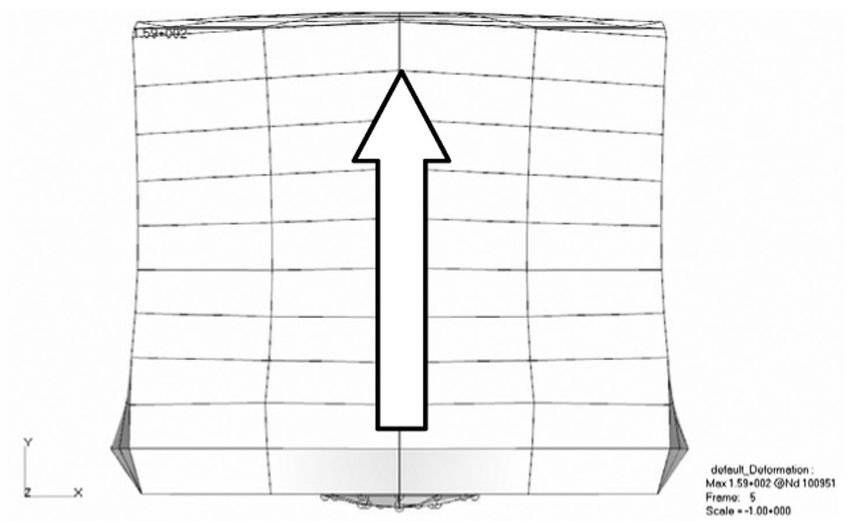

Fig. 9. TDA 6th mode: $1982 \mathrm{~Hz}-\mathrm{Y}$ translation (axial).

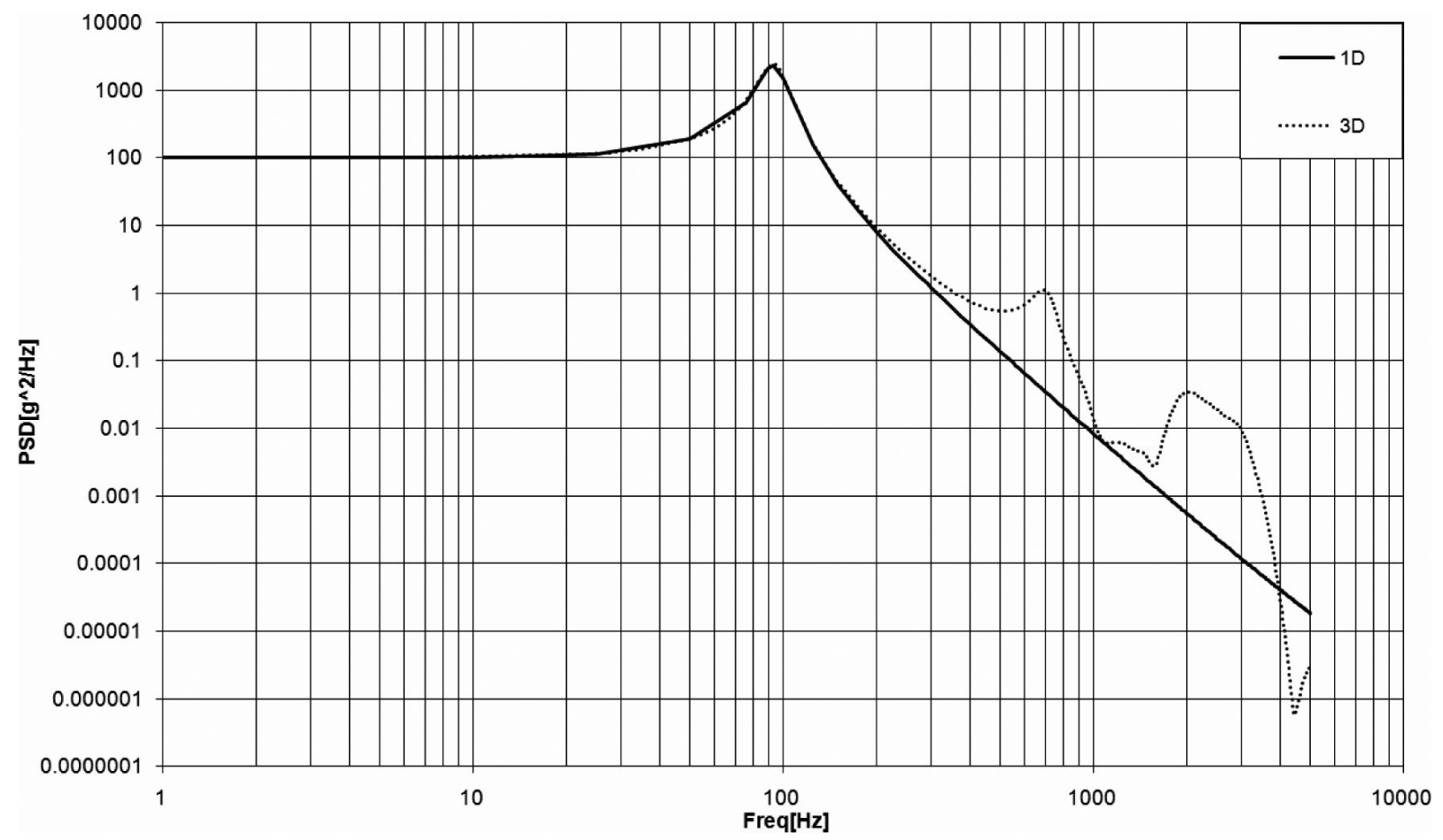

Fig. 10. Frequency response curves of a 1D TDA (dotted) and 3D TDA (solid).

$$
v_{\Omega} \triangleq v(x, y, z)
$$

Hence the volume velocity can be calculated using:

$$
V=\oiiint_{\Omega} v(x, y, z)
$$

The volume velocity is a quantitative measure of the structure acoustic radiation. The lower the volume velocity the weaker the noise the structure radiates [3].

Therefore, criteria of the numerical optimization procedure, intended to reduce the noise radiation relies on the term:

$$
\min \left(\oiint_{\Omega} v(x, y, z)\right)
$$



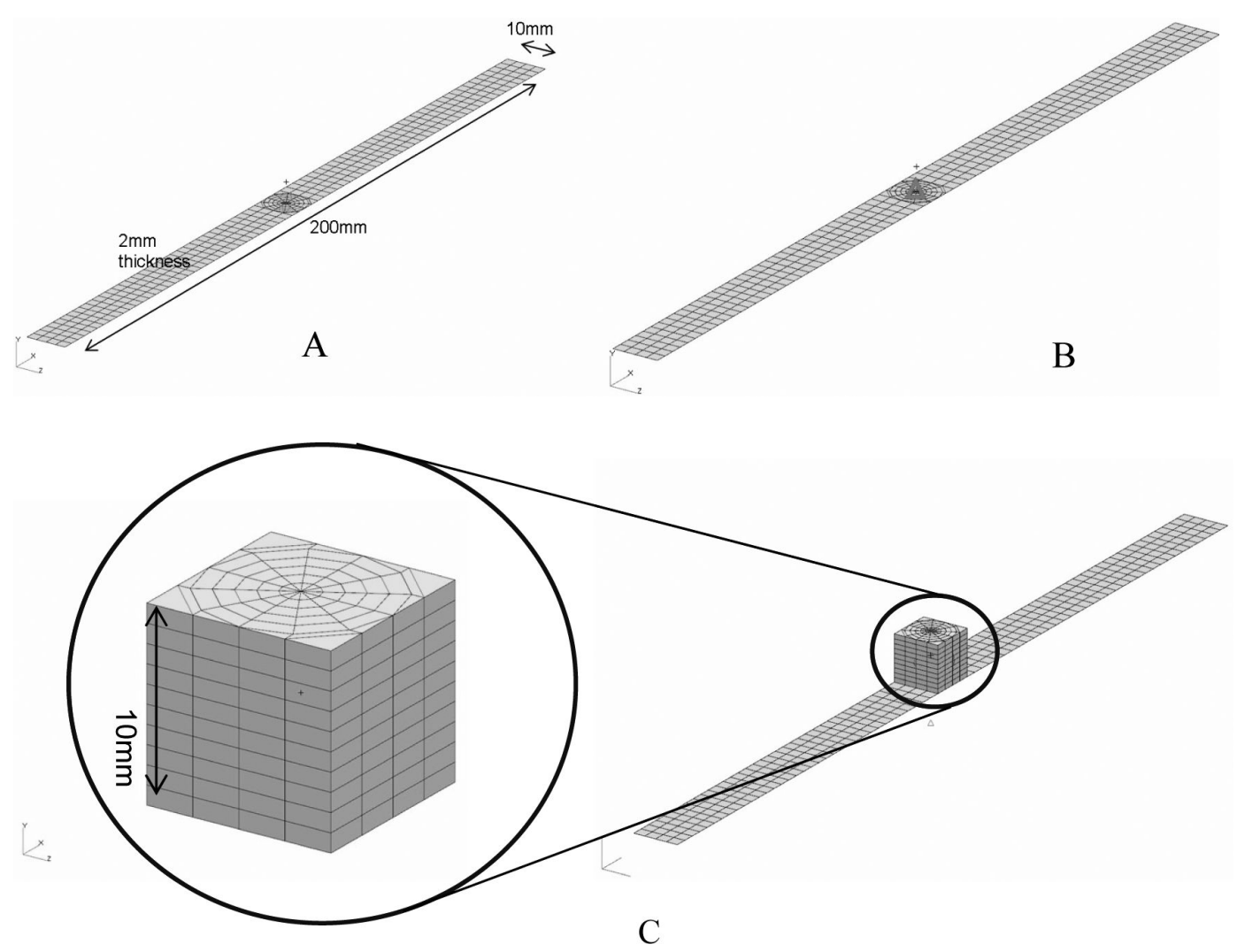

Fig. 11. (a) three cases of simply supported beam: A - Stand alone beam, B - Beam with a 1D TDA; (b) Three cases of simply supported beam: $\mathrm{C}$ - beam with 3D TDA.

The above definitions will be used in the next sections to evaluate the noise reduction capacity of different dynamic absorptions methods.

\subsection{Beam vibration reduction using $1 D$ and $3 D$ tuned dynamic absorbers}

The impressive wide spectrum dynamic response of the 3D TDA mentioned above and its wide spectrum absorption capacity are examined here using a simple dynamic system. A simply supported beam subjected to a frequency response procedure is evaluated in three distinctive cases. Firstly, the natural vibration of the beam on its own, secondly, a 1D TDA is attached at the midpoint of the beam and finally a 3D TDA is attached instead of the 1D TDA at the same midpoint of the beam. Each case had generated a different frequency response spectrum. The quantity measured here is the beam Volume Velocity at each frequency. Using the Volume Velocity as a criteria enables to take into account the continuous system overall response rather than a limited domain of the system. The FE model used to simulate the three cases above is illustrated in Fig. 12.

Figure 11A illustrates the stand alone $10 \times 200 \times 2 \mathrm{~mm}$ steel beam with a provision to a 1D TDA and a 3D TDA at its midpoint. A 1D TDA is added to the beam in Fig. 11B simply by adding a spring element with damping effect and a concerted $10 \mathrm{~g}$ mass. The spring element axial stiffness is stet to $K=3197[\mathrm{~N} / \mathrm{m}]$ in order to tune the $1 \mathrm{D}$ TDA to a $90 \mathrm{~Hz}$ resonance. The 3D TDA that is supplemented to the beam instead of the 1D TDA is illustrated at Figure 11C. Figure 11C also illustrates a zoom view of the 3D TDA with a structure of the 3D TDA similar to Fig. 5. The current 3D TDA consists of a $10 \mathrm{~mm}$ ball diameter with a density of Tungsten, $\rho_{\text {tung }}=19300\left[\mathrm{~kg} / \mathrm{mm}^{3}\right]$. The 3D TDA 6th mode (axial mode) was also tuned to a $90 \mathrm{~Hz}$ resonance. The frequency response curves generated from the three cases can be seen in the Fig. 12.

Figure 9 clearly verifies the enhanced vibration absorption capacity the 3D TDA has over the 1D TDA. It is evident that the enhanced vibration attenuation of the 3D TDA governs the whole spectrum. Moreover, meticulously 


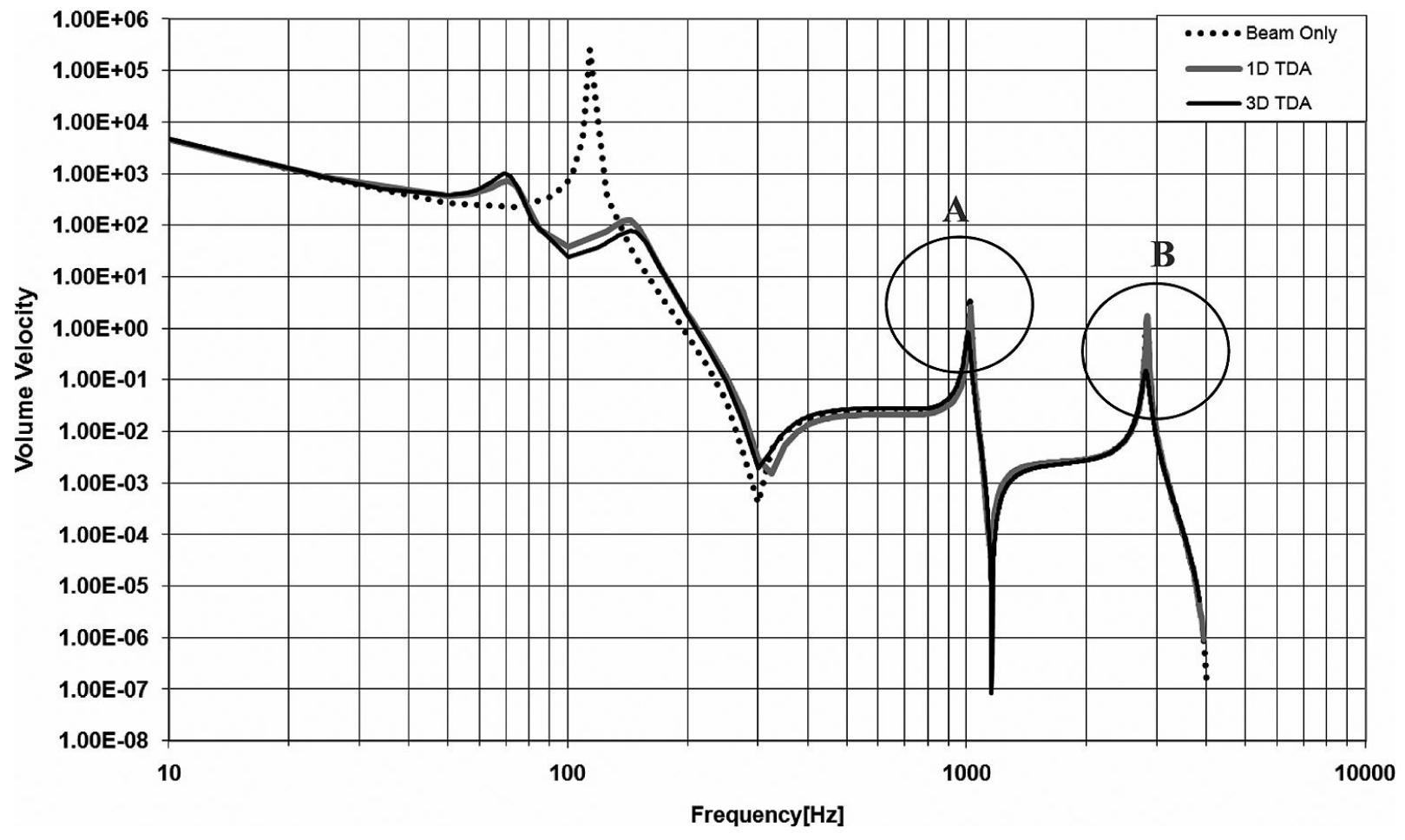

Fig. 12. Frequency response curves for three cases of simply supported beam. Stand alone beam (dotted), beam with added 1D TDA (grey), beam with added 3D TDA (black).

examining the frequency response curves at the high frequency peaks (circled in Fig. 12), revels that the 3D TDA vibration absorption capacity increases with frequency. This enhanced absorption is quantified in Fig. 13 which is a close-up views of the two peaks circled in Fig. 12.

Comparing the vibration attenuation at the two peaks reveals that the capacity rose from $80 \%$ at peak A to $92 \%$ at Peak B. It is also quantitatively substantiated in Fig. 13 that the absorption potential of the 3D TDA is much more impressive than the 1D TDA.

\section{Multiple cell 3d dynamic absorber}

The previous section was dedicated to the introduction of the 3D TDA and its predominance over the 1D TDA as a dynamic absorber. It is important to stress that the advantage that the 3D TDA has over the classic 1D TDA is stemming from its several normal modes. Having that in mind, one could ask: "what if we could multiply the amount of modes a 3D TDA has? Could we achieve the same (or better) absorption potential using the same (or less) volume and mass penalty? How imperative is it that the 3D TDA should be a multi modal TDA?" This section will be dedicated to answer the first question above. The two remaining questions will be answered at the next section.

The first question is answered by utilizing a basic feature of the 3D TDA conceptual model, presented in the previous section. This model can be considered as a single cell 3D TDA. This conceptual model allows the advantage of easily joining a number of 3D TDA cells to achieve a dynamic structure with multiplied normal modes, making it a multi modal 3D TDA. The section ahead will show two examples of a multiple cell 3D dynamic absorber and its modal analysis.

\subsection{Quadruple cell 3D dynamic absorber}

An interesting example of a multiple cell dynamic absorber would be a Quadruple cell dynamic absorber. The Quad-cell TDA is consisting of four unit cells joined together symmetrically as shown in Fig. 14. These Unit cells also consist of a $3 \mathrm{~mm}$ diameter ball embedded in the same $3.2 \mathrm{~mm}$ by $3.2 \mathrm{~mm}$ by $3.2 \mathrm{~mm}$ viscoelastic matrix. 

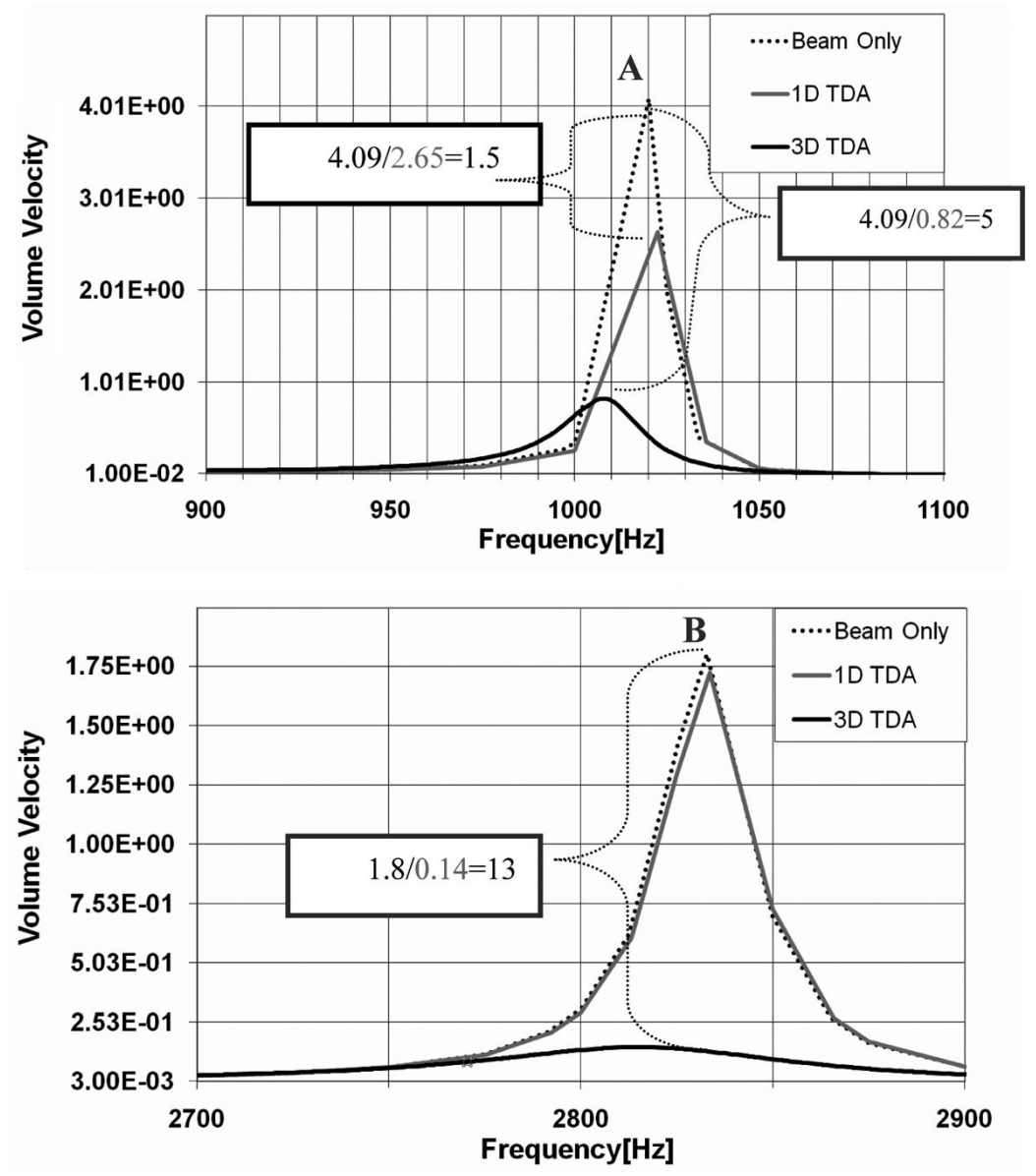

Fig. 13. Close-up view of the two resonance peaks from Fig. 12 Upper figure - Peak A, Lower figure - Peak B.
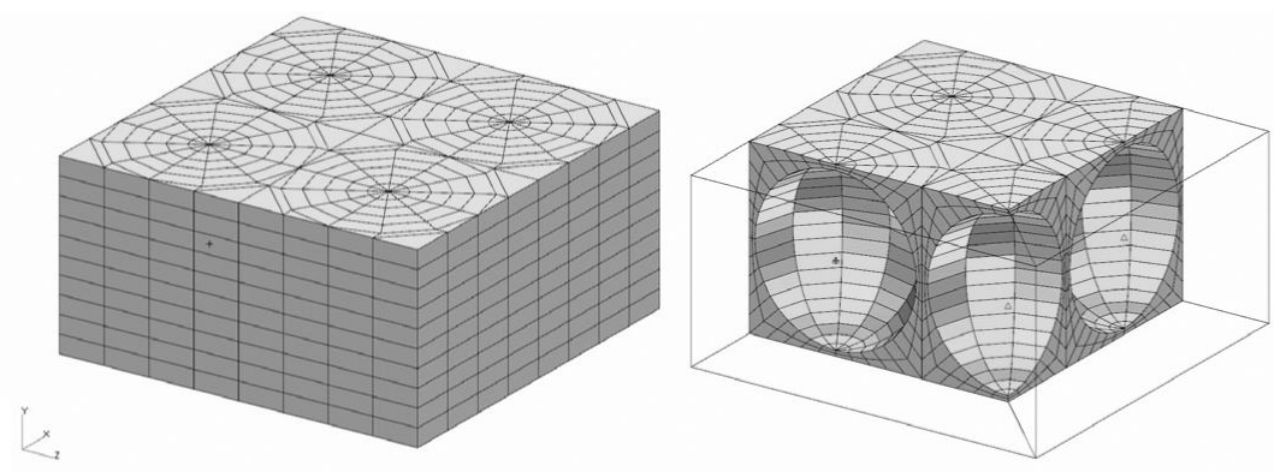

Fig. 14. Quadruple Cell 3D dynamic absorber.

\subsubsection{Normal modes of a quadruple cell 3D dynamic absorber}

As before, a normal modes simulation was conducted while the Quad-Cell 3D dynamic absorber was constrained at its bottom face (X-Z plane). Figures 15 to 24 show the produced normal modes.

The thirteen modes described in Figs 15-24 are only a part of the modes generated by the normal mode simulation. In fact, the Quad-cell 3D TDA had generated nearly four times the amount of modes the single cell generated at the $1 \mathrm{~Hz}$ to $2200 \mathrm{~Hz}$ frequency band. It worth to mention here, that some of the quad cell modes resemble the 


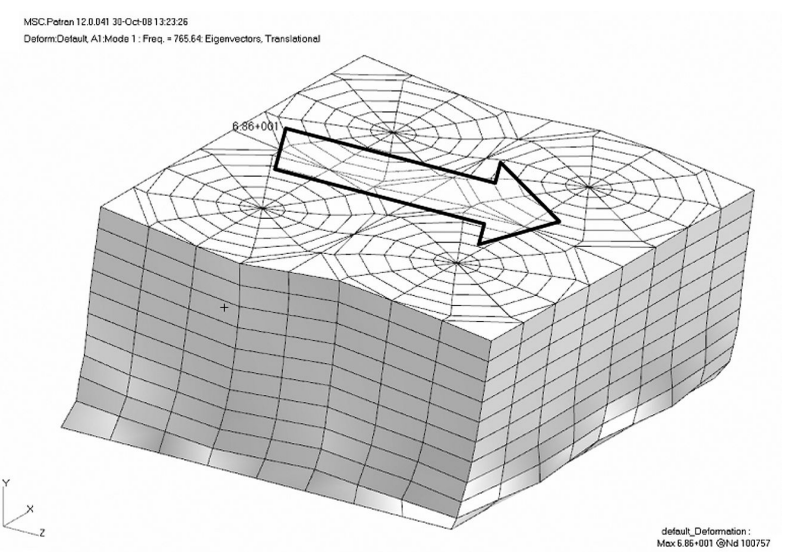

Fig. 15. 1st and 2nd mode: $765 \mathrm{~Hz}-\mathrm{X}, \mathrm{Z}$ Symmetric translation (single cell $641 \mathrm{~Hz}$ ).

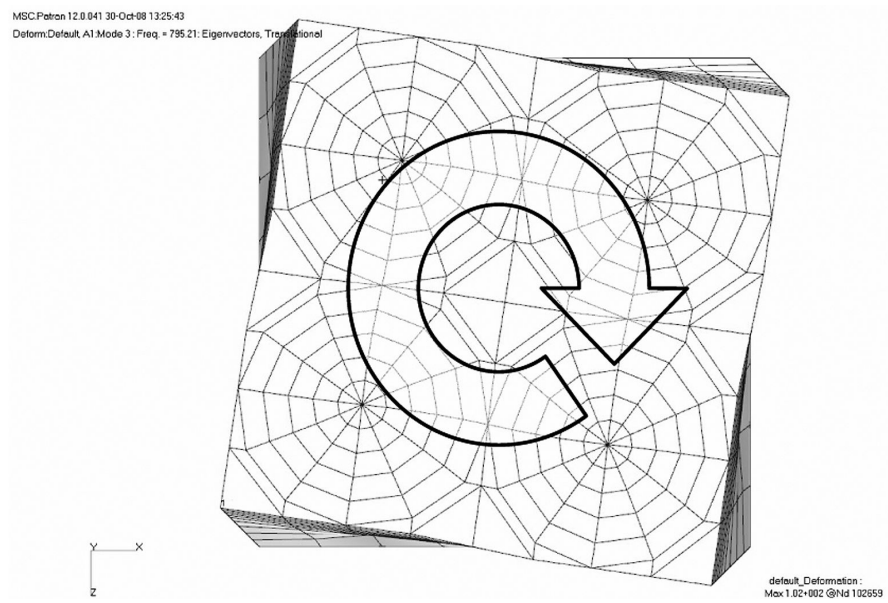

Fig. 16. 3rd mode : $781 \mathrm{~Hz}-\mathrm{Y}$ symmetric rotation.

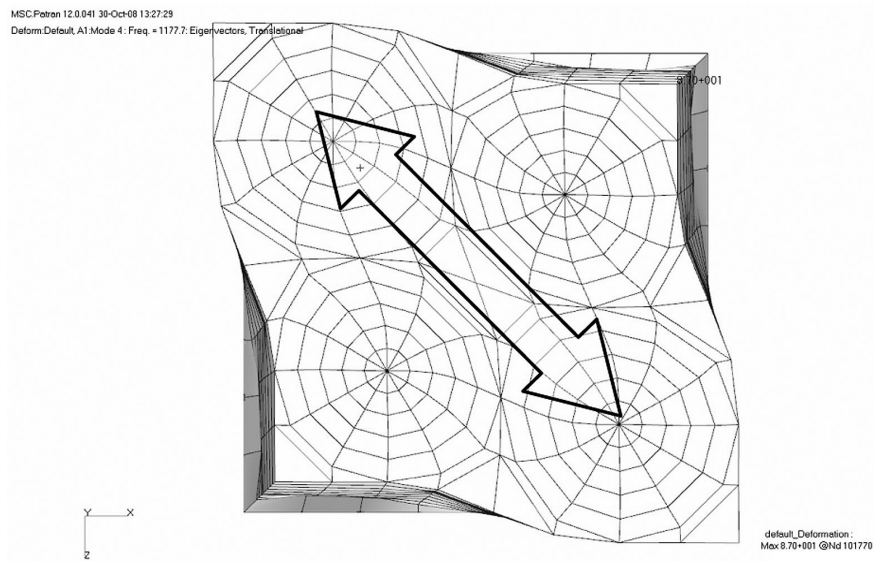

Fig. 17. 4th mode: $1177 \mathrm{~Hz}-\mathrm{X}-\mathrm{Z}$ in-Plane Shear. 


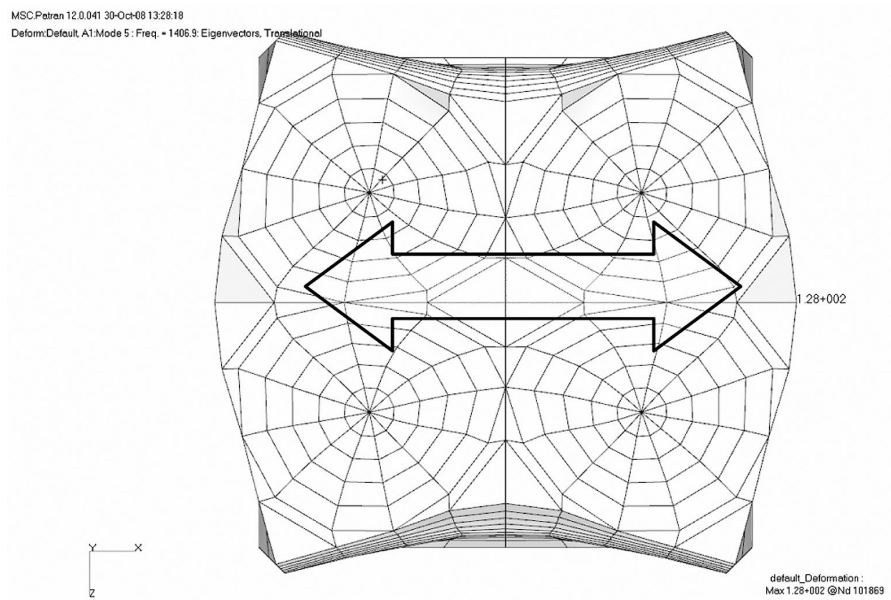

Fig. 18. 5th mode: $1407 \mathrm{~Hz}$.

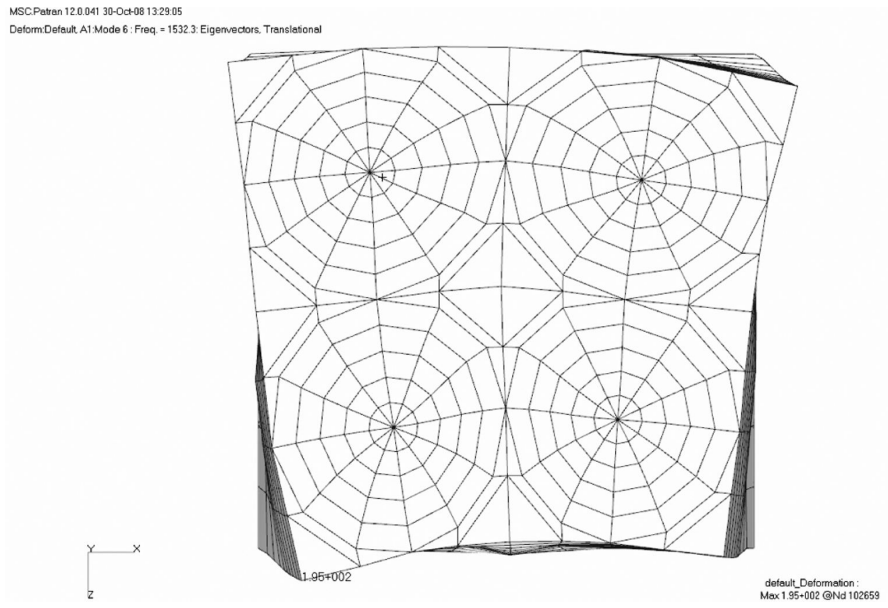

Fig. 19. 6th mode: $1532 \mathrm{~Hz}$.

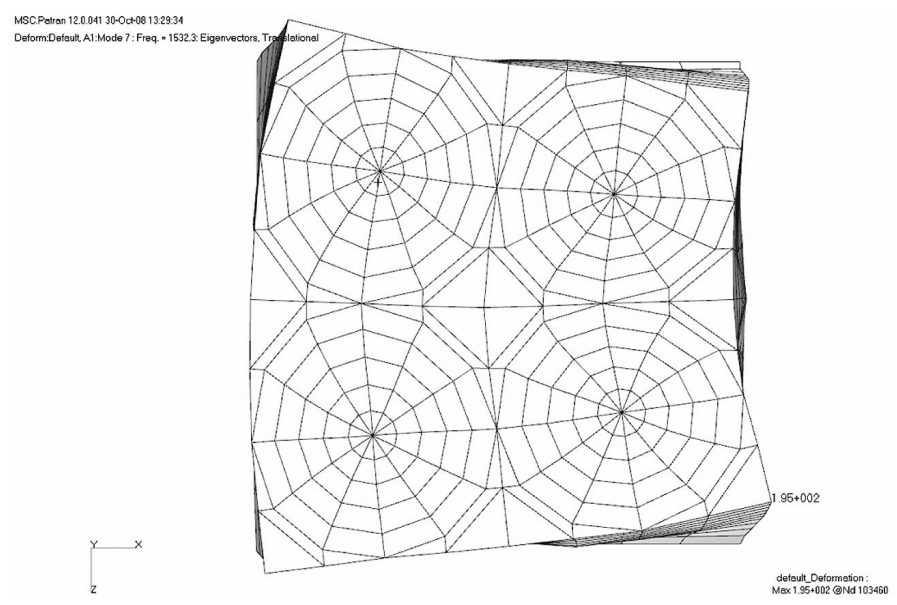

Fig. 20. 7th mode: $1532 \mathrm{~Hz}$. 


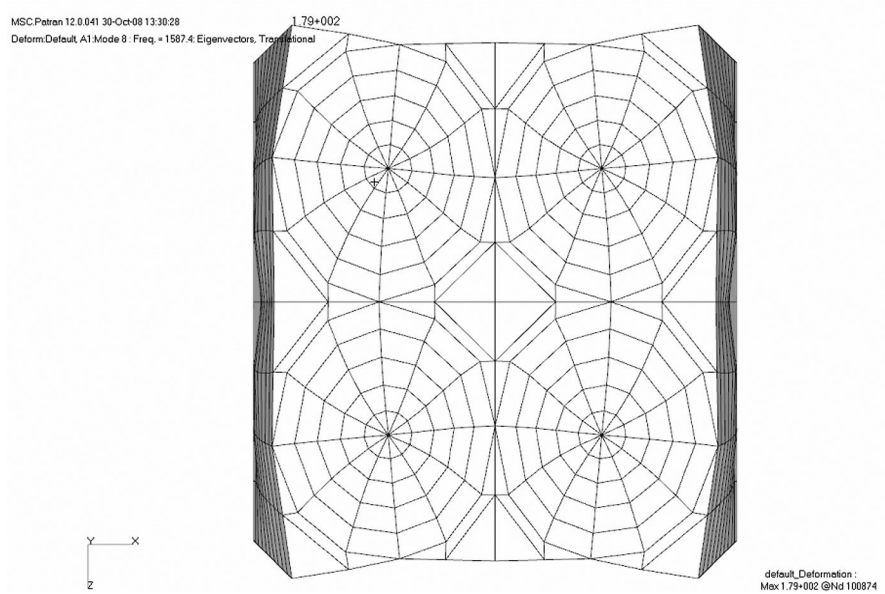

Fig. 21. 8th mode: $1587 \mathrm{~Hz}$.

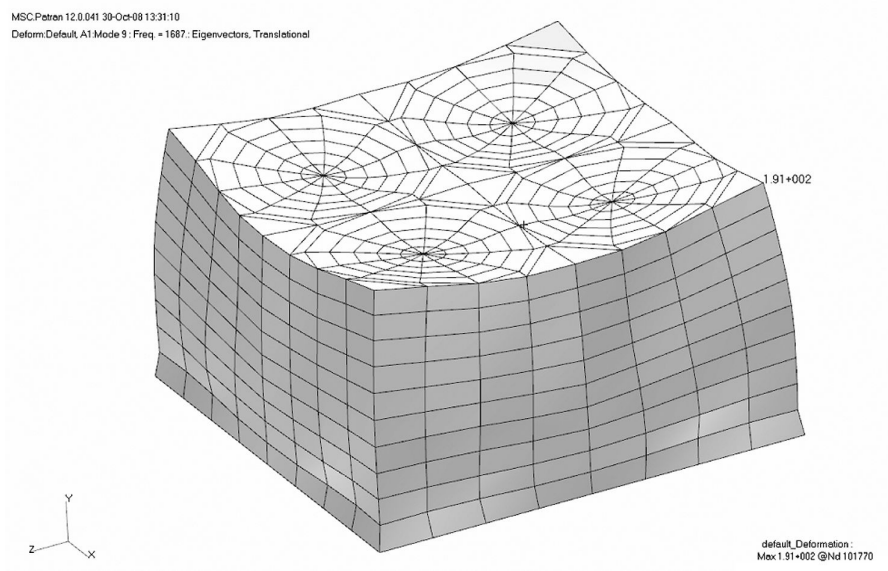

Fig. 22. 9th mode: $1687 \mathrm{~Hz}-\mathrm{Y}$ translation (axial) (single cell-1982 Hz).

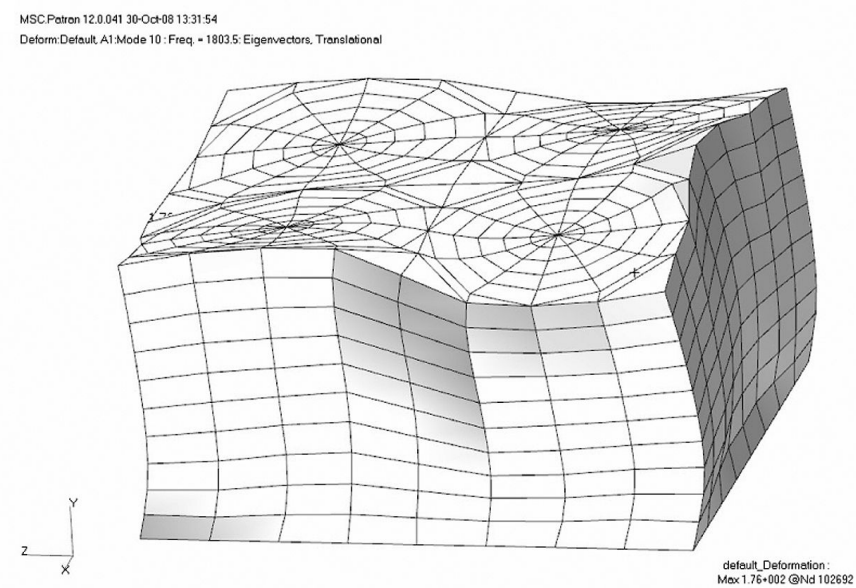

Fig. 23. 10th and 11th mode: $1803 \mathrm{~Hz}$. 


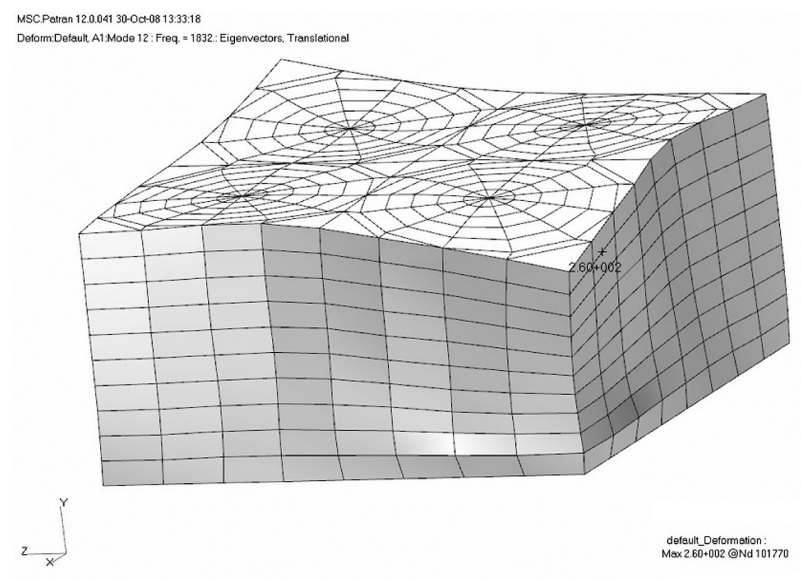

Fig. 24. 12th and 13th mode: $1832 \mathrm{~Hz}$.
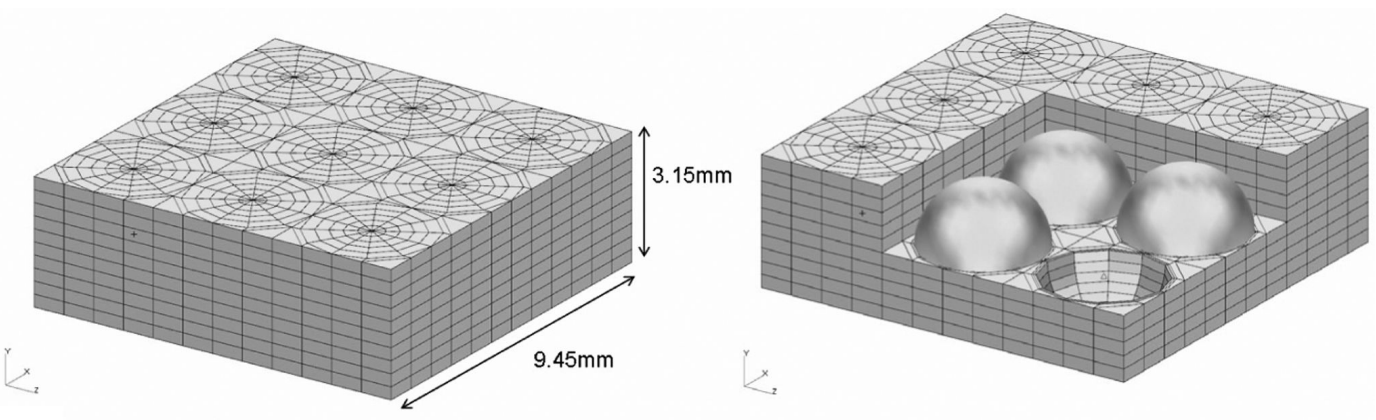

Fig. 25. Nona-cell dynamic absorber.

modes of the single cell, and some are completely new. Out of the new Quad-cell modes, there are many more that demonstrate an associate dynamic behaviour between the cells. This phenomenon will be even more evident in the Nine-cell 3D dynamic absorber introduced in the next section.

\subsection{Nona-cell 3D dynamic absorber}

The notion of multiplying the amount of modes causes a staggering effect in a Nona-cell (nine cells) dynamic absorber structure. The associated dynamic modes seen in the double and quad structures is an understatement in comparison to the dynamic behaviour seen in the Nona-cell dynamic absorber. Moreover, the usual axial mode shown at the previous examples is separated into several modes and therefore, defines a spectrum of axial response. This spectrum is also diverse in the intensity of dynamic absorbance due to the effect that the more cells involved with the mode the better the dynamic absorbance of the Nona-cell. The next Fig. 25 describes a Nona-cell dynamic absorber with nine $3 \mathrm{~mm}$ metal balls uniformly embedded in a $9.45 \mathrm{~mm}$ by $9.45 \mathrm{~mm}$ by $3.15 \mathrm{~mm}$ viscoelastic matrix.

\subsubsection{Normal modes of Nona-cell dynamic absorber}

As for the previous cases, a normal modes simulation was conducted while the Nona-cell dynamic absorber was constrained at its bottom face (X-Z plane). Figures 26 to 36 show the produced normal modes.

Figures 26 to 36 present a review of the main modes generated at the $1 \mathrm{~Hz}$ to $2000 \mathrm{~Hz}$ bound. Overall, an impressive amount of modes ( 32 modes) were generated in that bound for the Nona-cell dynamic absorber. There are some modes that are almost identical to the Quad-cell dynamic absorber, modes such as the 1st until the 4th modes, 7th, 8th and the 11th mode. In between those modes a few new modes are added in the Nona-cell configuration. All those modes involve a mutual dynamic response of the neighbouring cells. Two good examples are the 12 th 


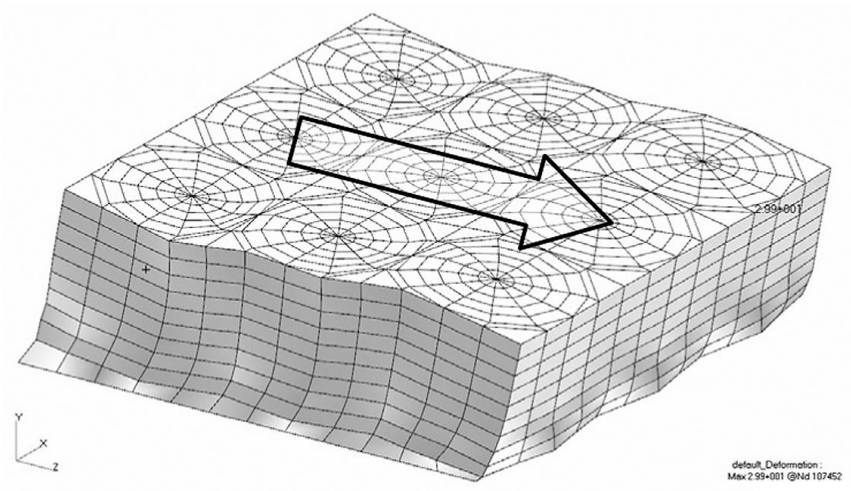

Fig. 26. 1st and 2nd mode: $-785 \mathrm{~Hz} \mathrm{X,} \mathrm{Z} \mathrm{Symmetric} \mathrm{translation} \mathrm{(785} \mathrm{Hz} \mathrm{Quad-cell).}$

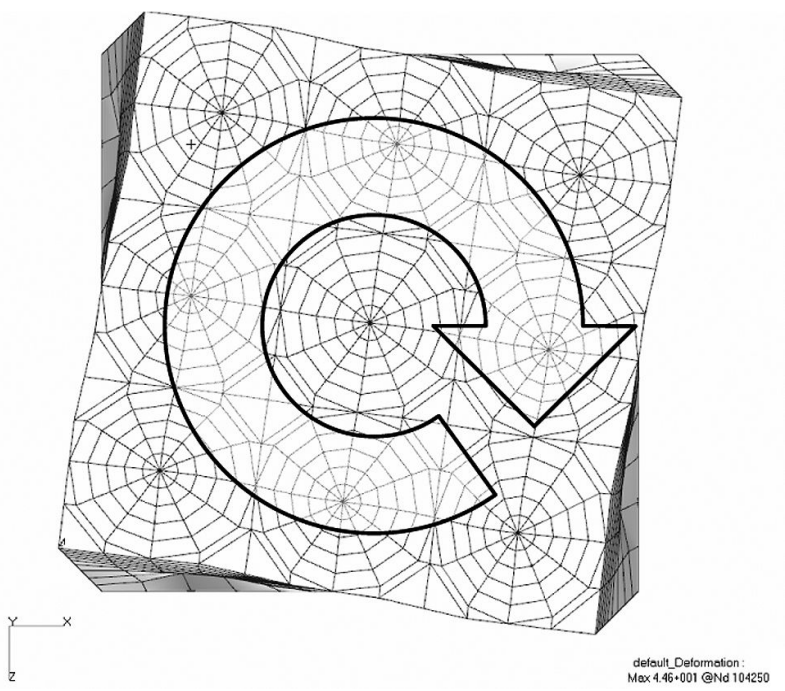

Fig. 27. 3rd mode: $-796 \mathrm{~Hz}$ Y Symmetric uniform rotation (781 Hz - Quad-cell).

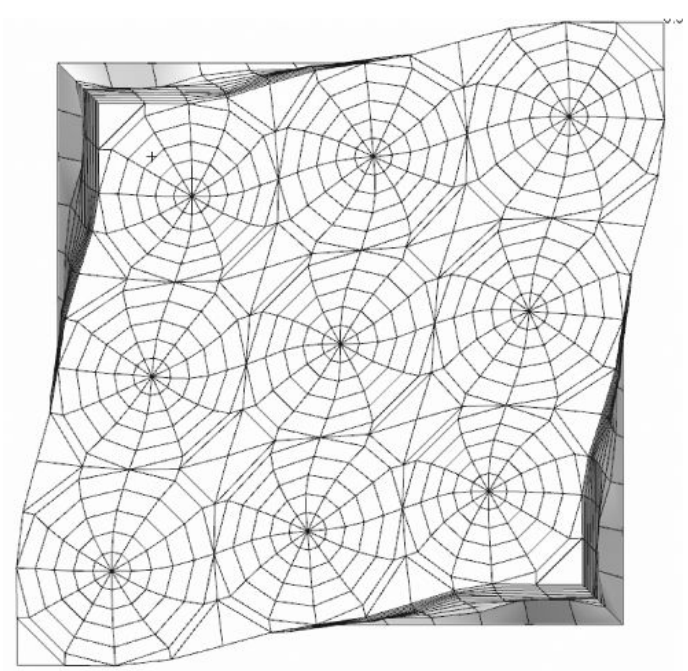

Fig. 28. 4th mode: $-995 \mathrm{~Hz} \mathrm{X,} \mathrm{Z} \mathrm{Symmetric} \mathrm{translation} \mathrm{(1177} \mathrm{Hz} \mathrm{-} \mathrm{Quad-cell).}$ 

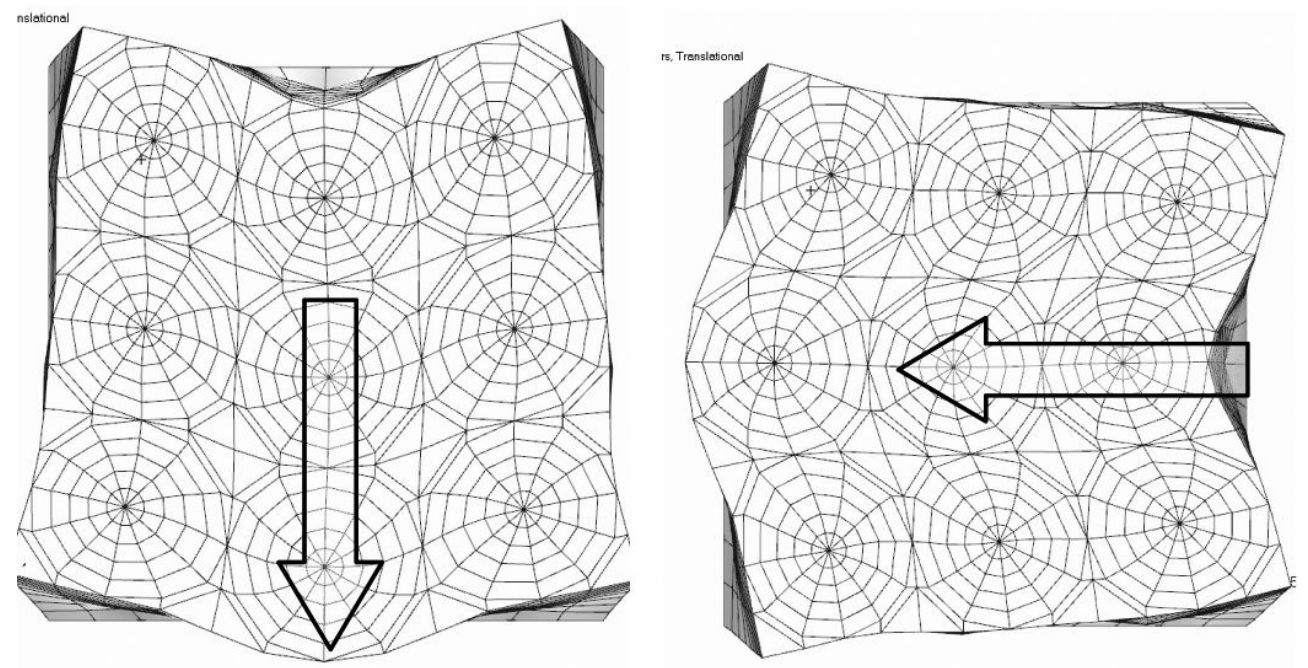

Fig. 29. 5th (left) and 6th (right) mode: $-1139 \mathrm{~Hz} \mathrm{X}, \mathrm{Z}$ none uniform translation.

(Fig. 34) and 15th (Fig. 35) modes, where mutual rotational and mutual transitional modes are shown respectively at the $1 \mathrm{~Hz}$ to $2200 \mathrm{~Hz}$ frequency band.

A unique phenomenon first seen in the Nona-cell dynamic absorber is the span of the axial modes into a spectrum of axial modes. This spectrum stretches on a wide $750 \mathrm{~Hz}$ range and consists of nine different modes, starting from the 15 th mode to the 36th mode. It can be seen from Fig. 36 that each axial mode has a unique behaviour where at each mode different cells are involved in the mutual dynamic response. Moreover, it can be seen that some modes involve more cells than others. This suggests that some modes are more intense than others.

The next stage of this work was to examine the vibration attenuation potential when the notion of multiplying the modes is taken to the extreme. This will be achieved by joining numerous cells in to a planar arraignment to create a layer of 3D TDA's.

\section{Elasto-inertial dynamic layer}

The preceding two sections were dedicated to the presentation, explanation and justification of the multi modal dynamic absorber. The benefits of using a multi modal dynamic absorber have been explained in detail. Nevertheless, it was also established that a beneficial supplementation of a multi modal dynamic absorber is not achieved through a straight forward method. Consequentially, a deep understanding of the mechanical system is needed to successfully design a suitable multi modal dynamic absorber.

This section is dedicated to the development of a wide frequency range and global dynamic absorption design concept. This design is a wide scale expansion of the concept of multi-cell dynamic absorber. At a nutshell, this concept consists of a large number of 3D unit cells arranged in a planar fashion. This structure is then attached to a surface of a mechanical entity to form a Multi modal dynamic absorbing layer. This concept will be referred as elasto-inertial dynamic layer (EIDL).

\subsection{The design of elasto-inertial dynamic layer}

Figure 37 visualizes the concept of the EIDL introduced in the previous section. As mentioned, this concept is essentially an expended multi modal dynamic absorber.

The section view of the EIDL revel its analogy to the multi modal dynamic absorber. The EIDL is constructed through a large number of metal balls embedded in a viscoelastic matrix, as shown in Fig. 38.

As suggested before, the EIDL can easily be attached to a planar entity such a plate as shown in Fig. 39 below. 


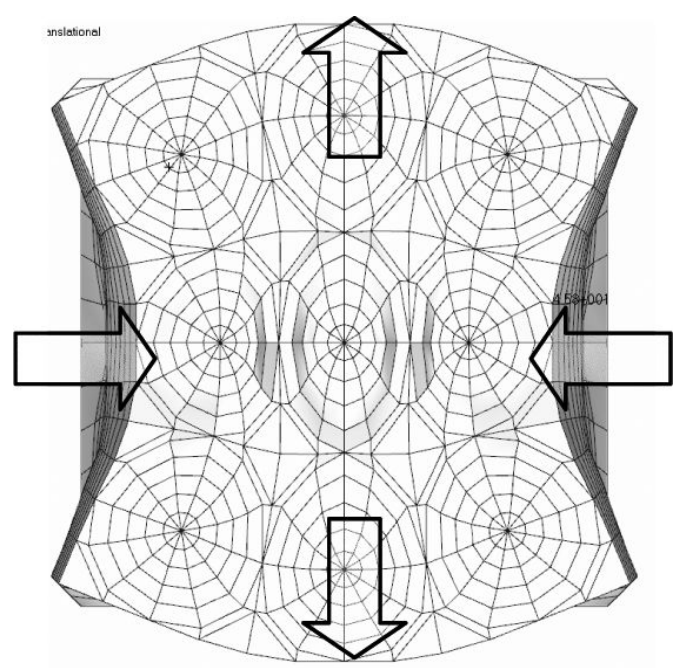

Fig. 30. 7th mode: $-1248 \mathrm{~Hz}(1407 \mathrm{~Hz}-$ Quad-cell).

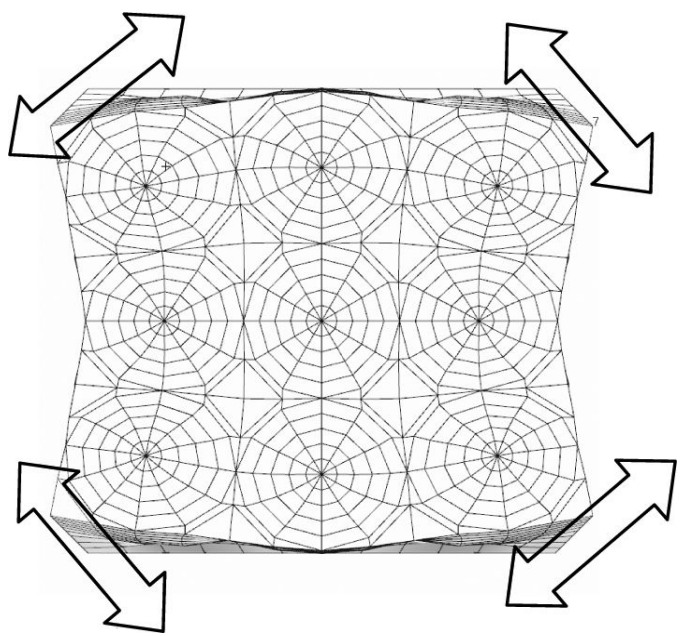

Fig. 31. 8th mode: $-1346 \mathrm{~Hz}(1587 \mathrm{~Hz}-$ Quad-cell).
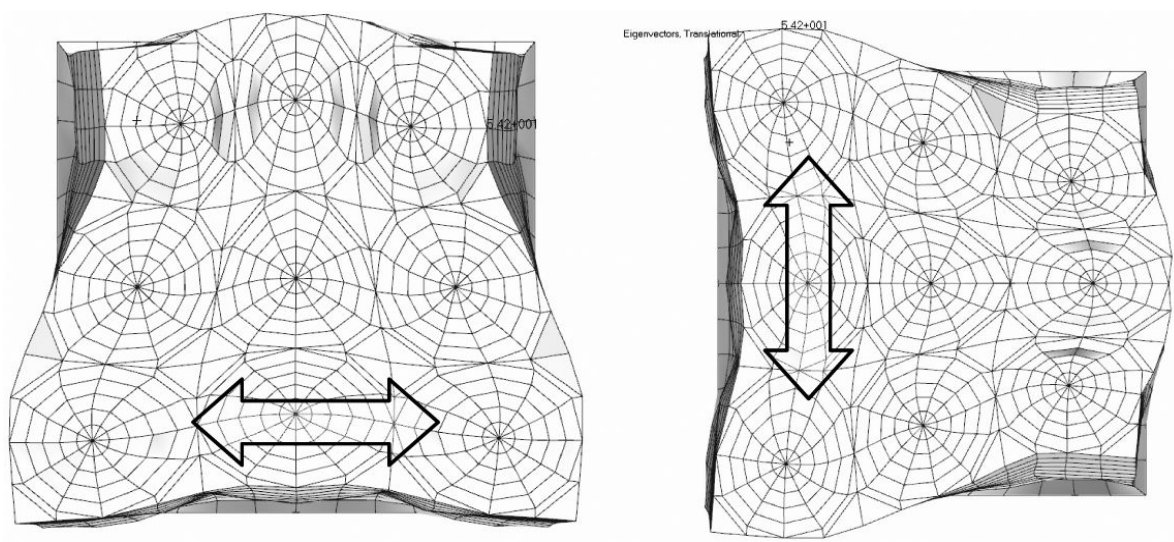

Fig. 32. 9th (left) and 10th (right) mode: $-1395 \mathrm{~Hz}$. 


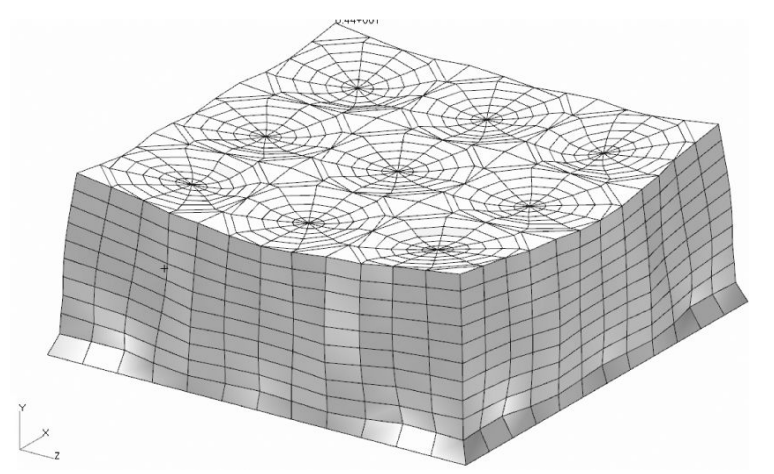

Fig. 33. 11th mode: $1471 \mathrm{~Hz}-$ First axial mode (1687 Hz-Quad-cell).

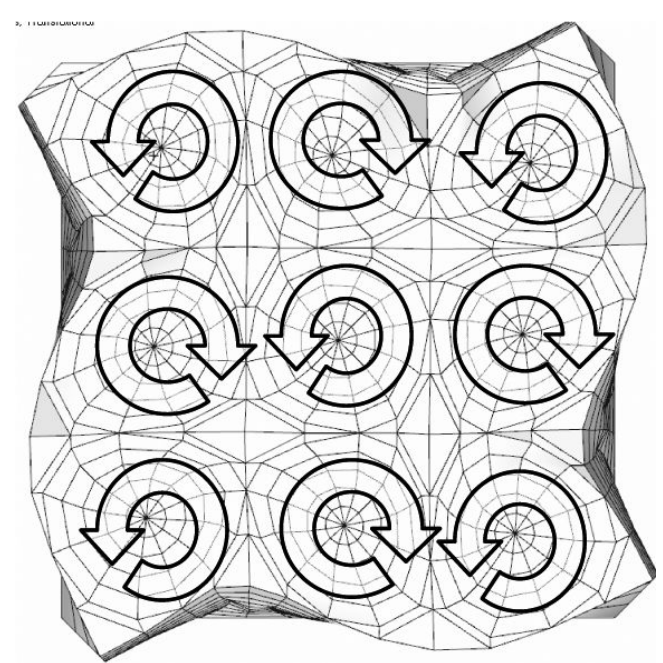

Fig. 34. 12th mode: $1535 \mathrm{~Hz}$.

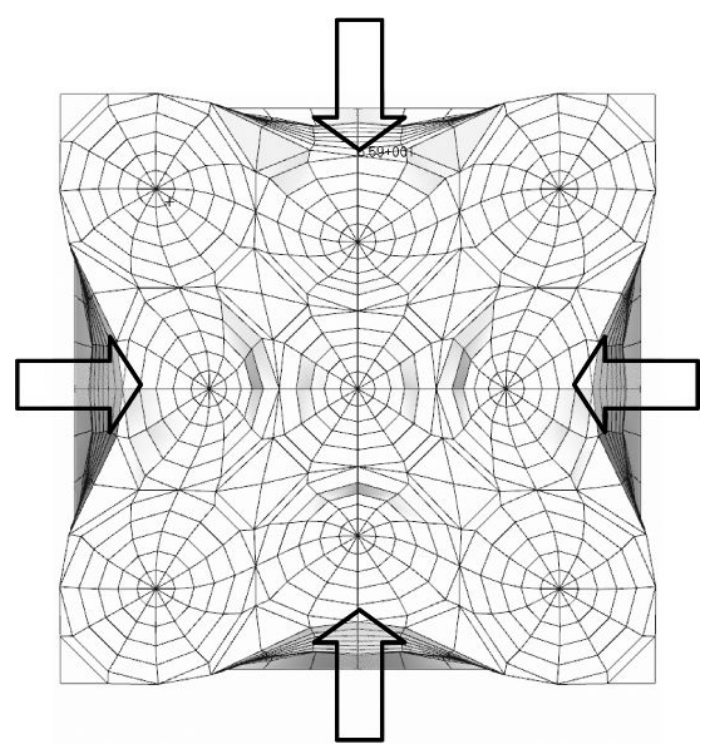

Fig. 35. 15th mode: $1700 \mathrm{~Hz}$. 
$16^{\text {th }}$

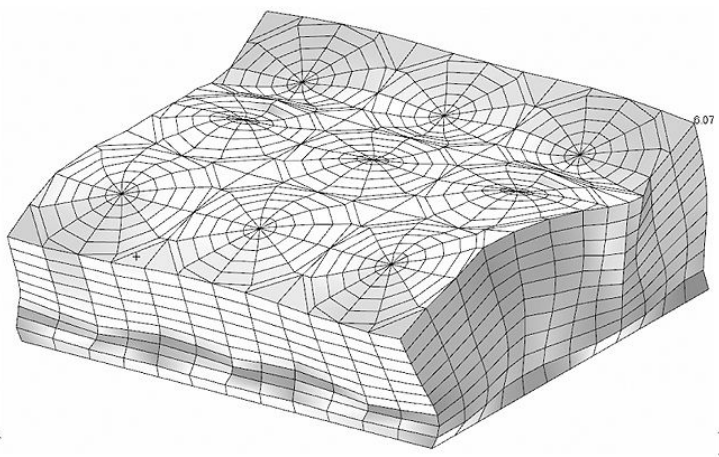

$19^{\text {th }}$

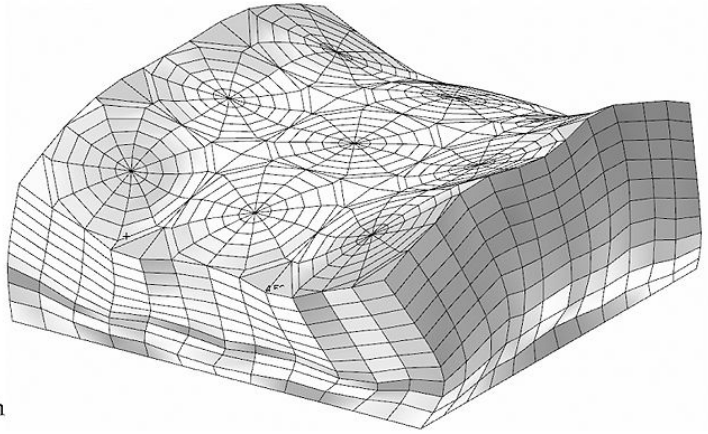

$24^{\text {th }}$

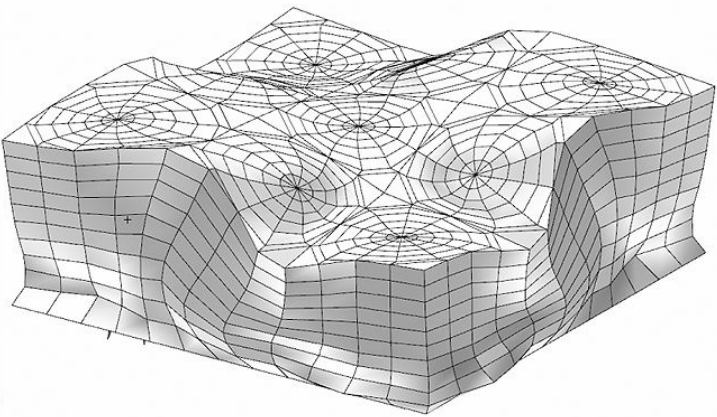

$21^{\text {st }}$
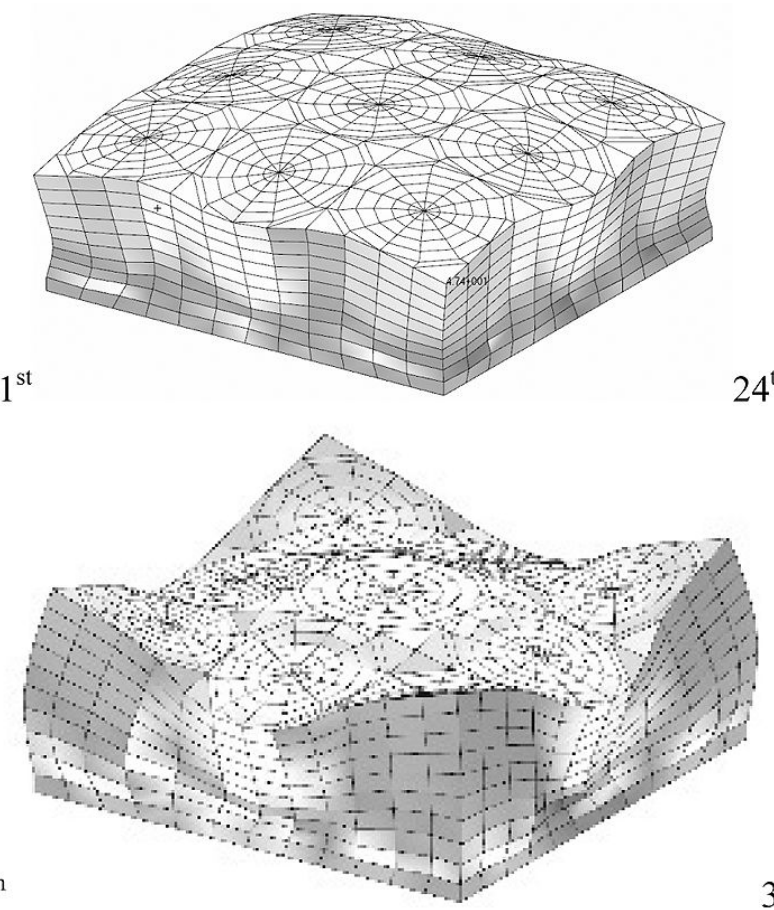

$29^{\text {th }}$

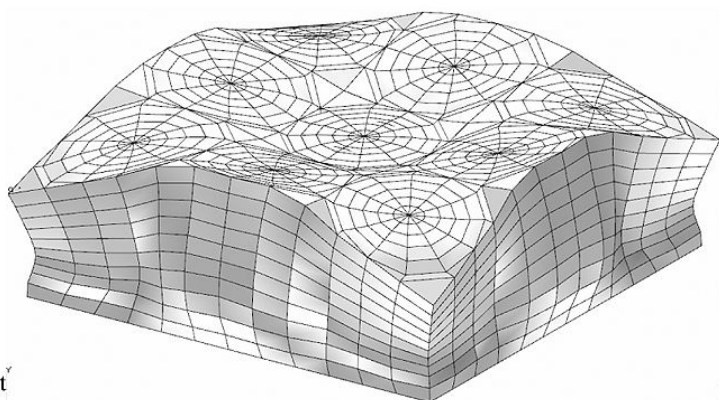

$31^{\text {st }}$

$30^{\text {th }}$

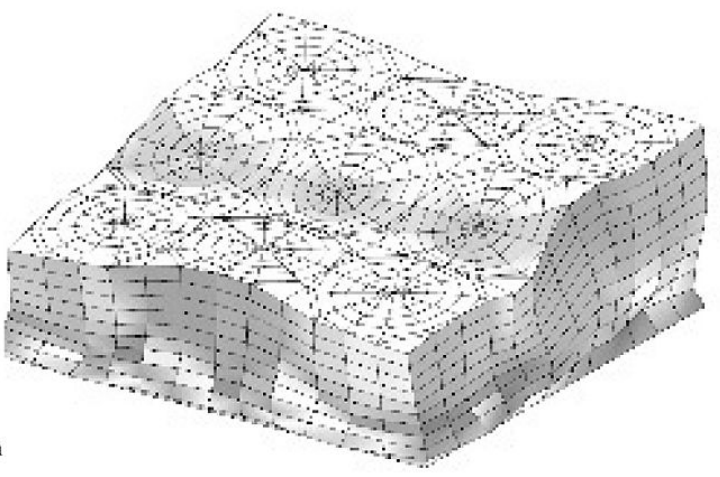

$36^{\text {th }}$

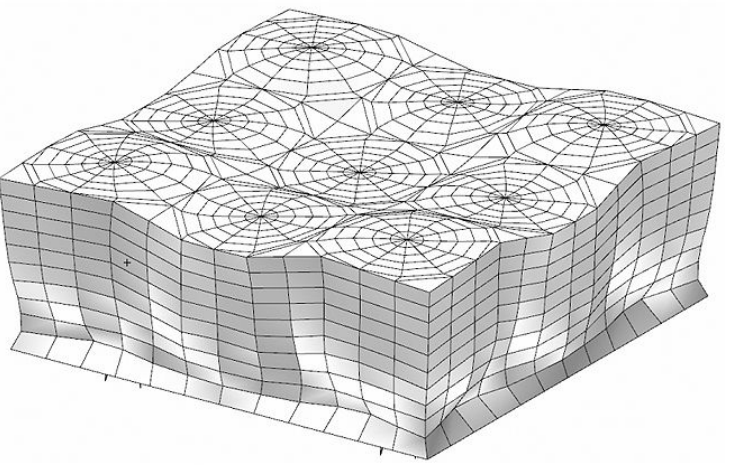

Fig. 36. Eight axial translation modes: $1744 \mathrm{~Hz}$ to $2222 \mathrm{~Hz}$. 


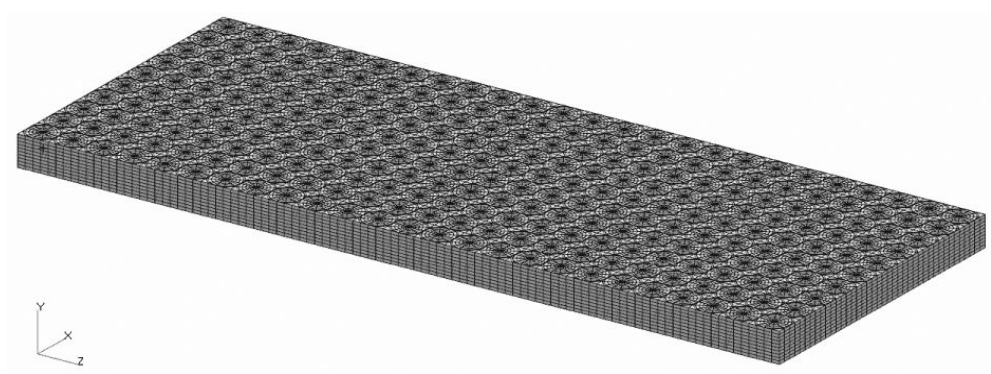

Fig. 37. Elasto-Inertial Dynamic Layer.

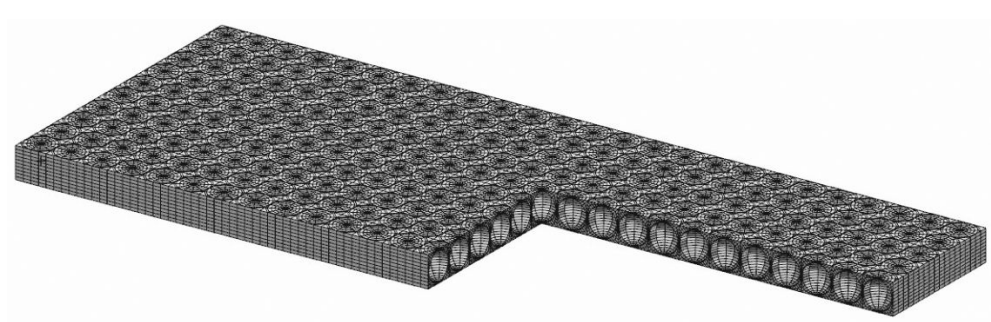

Fig. 38. Section view of the Elasto-Inertial Dynamic Layer.

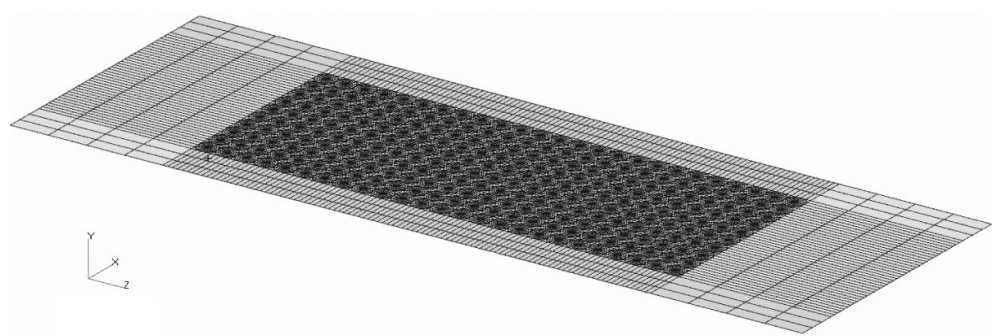

Fig. 39. Elasto-Inertial Dynamic Layer attached to a plate.

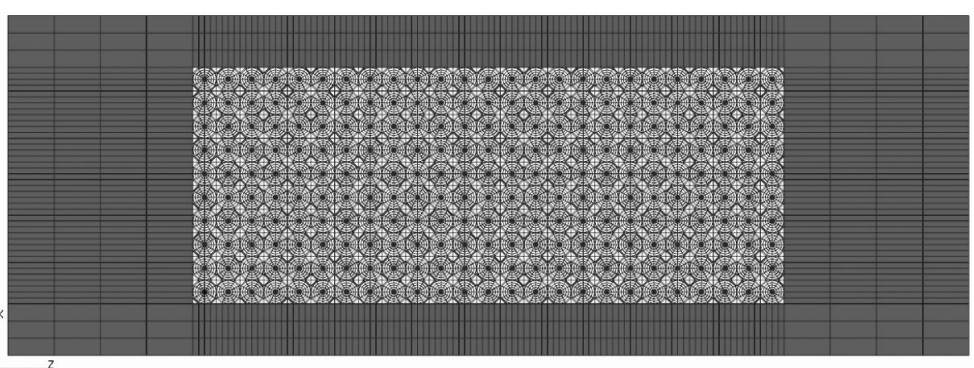

Fig. 40. A $46 \mathrm{~mm}$ by $130 \mathrm{~mm}$ aluminium plate with variable thickness Perimeter $-7 \mathrm{~mm}$ thickness, Core- $3 \mathrm{~mm}$ thickness.

\subsection{Normal modes of a plate with an elasto-inertial dynamic layer}

In order to visualize the dynamic properties of the EIDL, a simple normal mode FE simulation was conducted on the plate shown in Fig. 39. The plate used is a $46 \mathrm{~mm}$ by $130 \mathrm{~mm}$ aluminium plate with variable thickness as shown in Fig. 40. The EIDL, located at the centre of the plate, consists of 25 by 10 cells based on the $3.2 \mathrm{~mm}$ unit cell shown in Fig. 5. Each cell contains a $3 \mathrm{~mm}$ diameter steel ball. The simulation assumed free- free boundary conditions at the plate corners. 


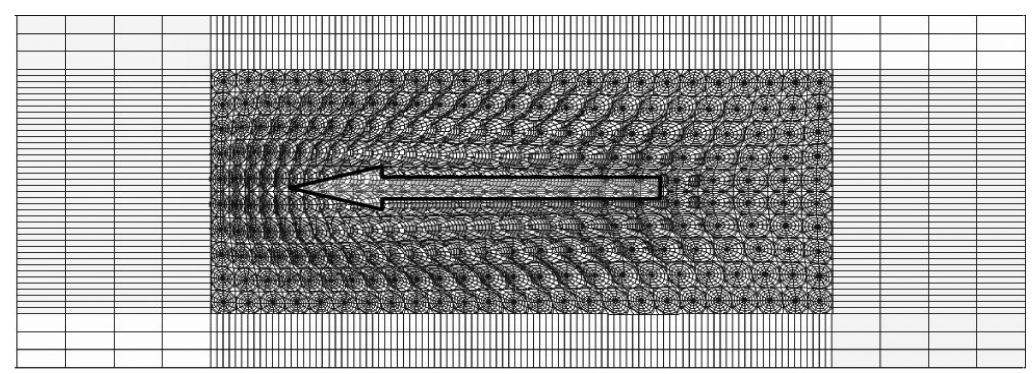

Fig. 41. EIDL 1st mode - $882 \mathrm{~Hz}$ - in plane uniform horizontal translation.

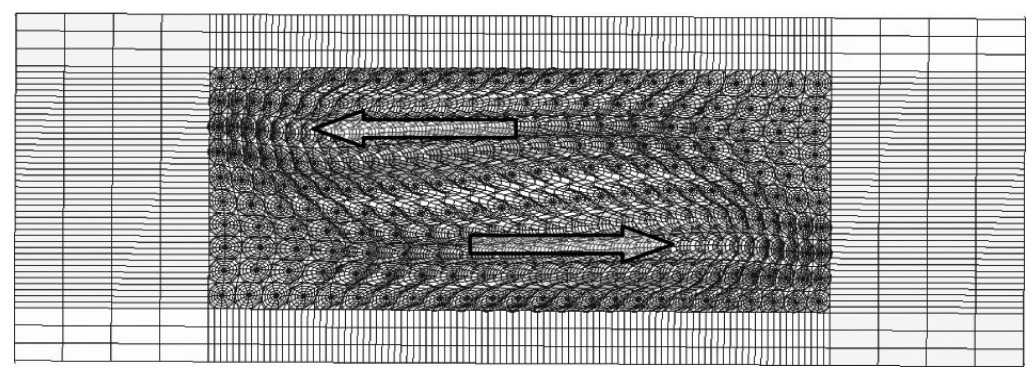

Fig. 42. EIDL 2nd mode $-907 \mathrm{~Hz}$ - in plane opposite horizontal translation.

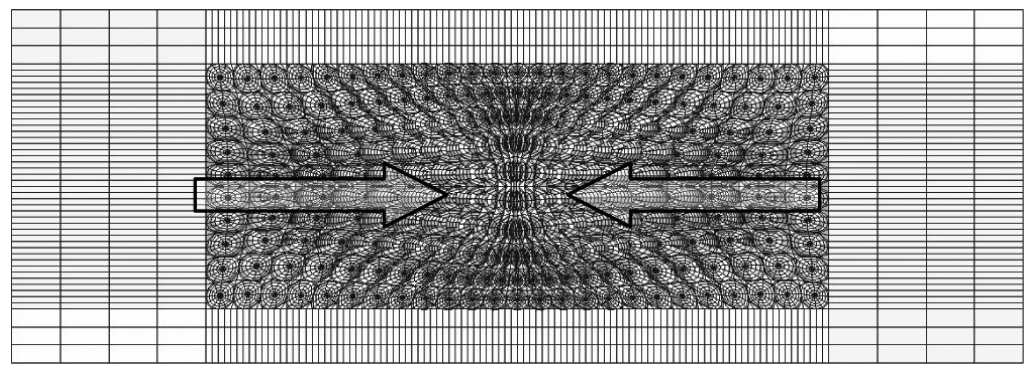

Fig. 43. EIDL 3rd mode $-908 \mathrm{~Hz}-$ in plane contradictory horizontal translation.

This simulation reviled a remarkable dynamic behaviour some are analogous to the aforementioned Quad-cell and Nona-cell dynamic absorbers and some are completely different. These modes are shown and explained in Figs 41 to 51 .

As seen at the previous section for Quad-cell and Nona-cell structure, the first mode of the EIDL is a uniform translation of the cells shown in Fig. 41.

A non uniform translation such as the opposite in plane translation shown in Fig. 42 was also seen at the Nona-cell fifth and sixth modes (see Fig. 29).

This mode of in plane contradictory horizontal translation is created when one half of the EIDL is moving in contrary to the other half. This mode is analogous to the Nona-cell seventh mode (see Fig. 30).

The EIDL forth mode is an interesting mode shape that involves a rotational translation of a number of cells. This mode is vaguely resembling the Nona-cell twelfth mode (see Fig. 34).

The next seven modes of the EIDL are a permutation of the previous basic four modes. Some of the forthcoming modes of the EIDL are a higher harmonic of a basic mode and some are a combination of two, three and even four of the basic modes. Furthermore, it's important to emphasize the fact that all of EIDL modes are densely spread along the frequency range. 


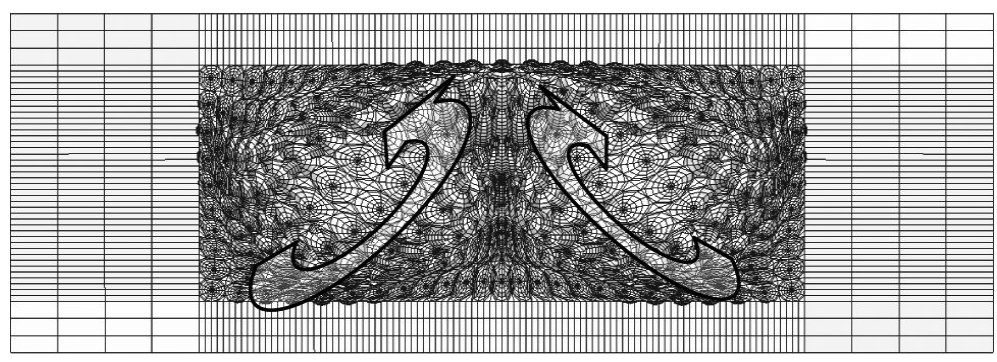

Fig. 44. EIDL 4th mode $-922 \mathrm{~Hz}-$ in plane swirl translation.

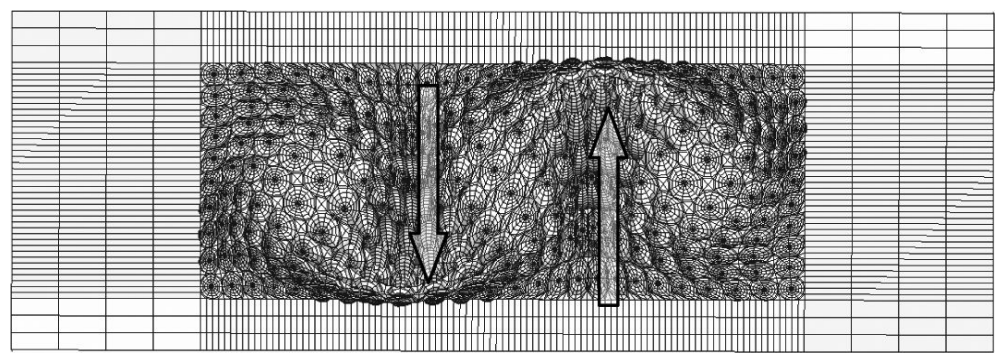

Fig. 45. EIDL 5th mode $-937 \mathrm{~Hz}-$ in plane opposite vertical translation.

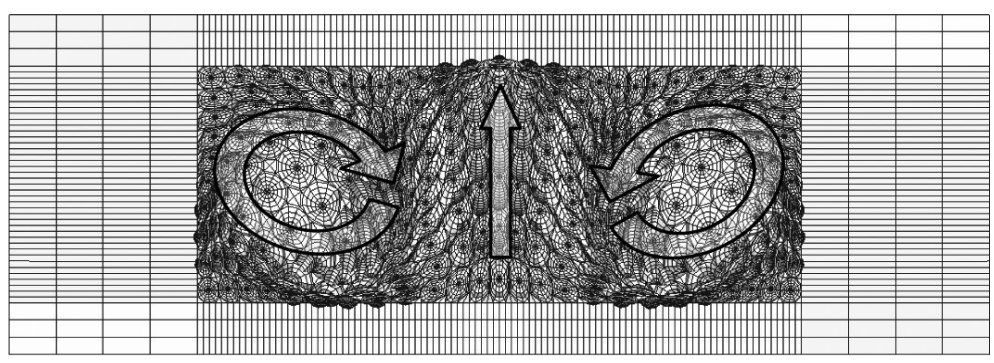

Fig. 46. EIDL 6th mode $-956 \mathrm{~Hz}$ - in plane swirl and horizontal translation combination.

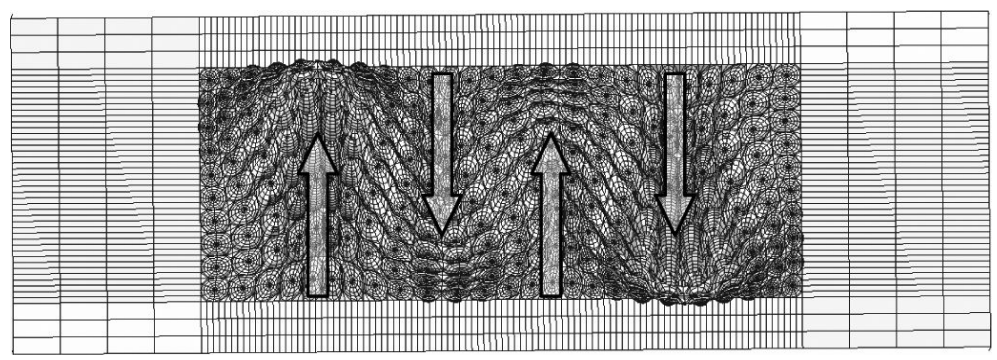

Fig. 47. EIDL 7th mode $-965 \mathrm{~Hz}$ - in plane quadruple opposite vertical translation.

\subsection{Plate vibration attenuation using elasto-inertial dynamic layer}

The promising dynamic behaviour, shown by the EIDL normal modes at the previous section, is brought to the test of vibration attenuation in a simple mechanical system. This system, shown in Fig. 52 is exactly the same plate with EIDL presented in Fig. 40, except for an added mass rigidly connected to its centre. This mass, set to $700 \mathrm{gr}$, represents a vibrating electric motor. 


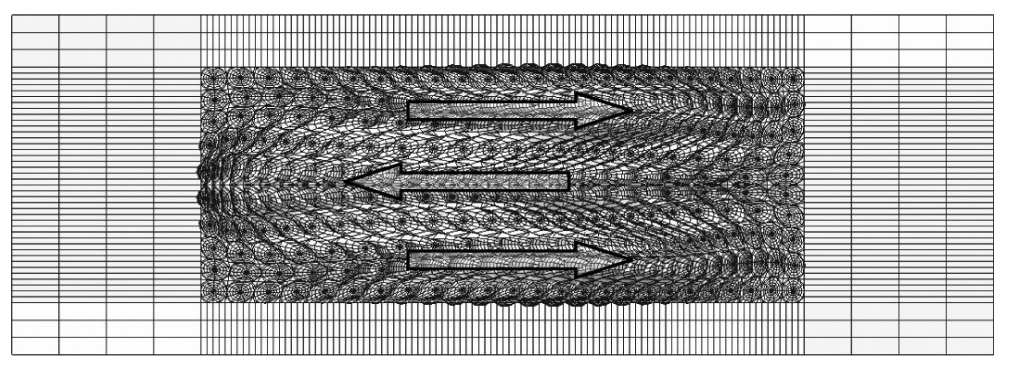

Fig. 48. EIDL 8th mode $-971 \mathrm{~Hz}$ - in plane triple opposite horizontal translation.

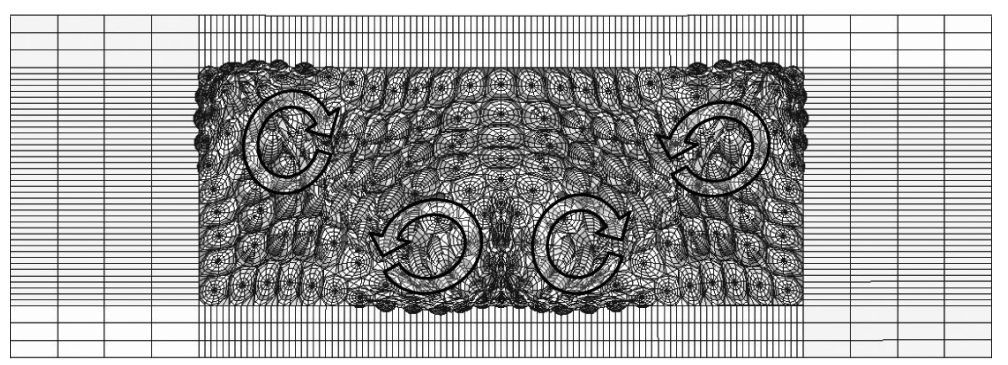

Fig. 49. EIDL 9th mode $-974 \mathrm{~Hz}$ - in plane quadruple opposite swirl translation.

The model shown in Fig. 52 represents a typical mechanical system with a source of wide range vibration export. It is consisted of a structure (plate) and a vibration inducer (unbalanced motor). It is imperative that the vibration export by the motor will be amplified as little as possible by the plate to minimize avoid noise radiation. Therefore, the aim of the EIDL is to attenuate the vibrations transferred to the plate by the unbalanced motor as much as possible.

A frequency response simulation is conducted with the system above where the motor is subjected to a sinusoidal excitation at a direction normal to the plate. This simulation was made twice, one with EIDL and one without. The results for the frequency response curves are given in Fig. 53.

As it vividly shown from Fig. 53, the EIDL contributes to the overall vibration attenuation of the plate. From the very low frequency bound to the far end of the simulation range. The wide range excitation resulted in the excitation of hundreds of modes of the EIDL, with as little as few $\mathrm{Hz}$ separating one another. Once again, the existence of a multi modal dynamic absorber entity such as the EIDL proves to be effective in vibration attenuation. This effective performance is achieved with a negligible added mass penalty of $27 \mathrm{gr}$ that is less than $4 \%$ of the overall mass of the model $(800 \mathrm{gr})$. A sensible explanation to how do the existence of the many in plane modes influence the normal dynamic response of the plate is given in the next section.

\subsection{Vertical mode reshaping}

The previous two sections reviled the dynamic behaviour of the EIDL and its vibration attenuation potential. As shown in Section 4.2, the EIDL normal modes can be characterized as in-plane modes. It was established earlier, that the modal reshaping the dynamic absorber inhabit, provides an effective method of vibration suppression. As appose to the classical dynamic absorber, where the resonant mode shapes of a structure are attenuated by a motion in parallel direction of a lumped mass, the attached EIDL motion (-modes) is perpendicular to the structure mode shapes. In other words, the attached EIDL allows vibration attenuation through a modal reshaping in the vertical direction of excitation. This phenomenon will be termed as vertical mode reshaping (VMR).

The approach of a self-damping system has introduced the existence of a wave pressure phenomenon [2]. This effect provides the key in understanding the influence of the VMR, on the plate normal dynamic response.

Figure 54 illustrates a plate with EIDL and two adjacent EIDL cells. The metal balls are represented by two grey circles and the viscoelastic matrix between them is represented by a mass-damper couple. In the absence of 


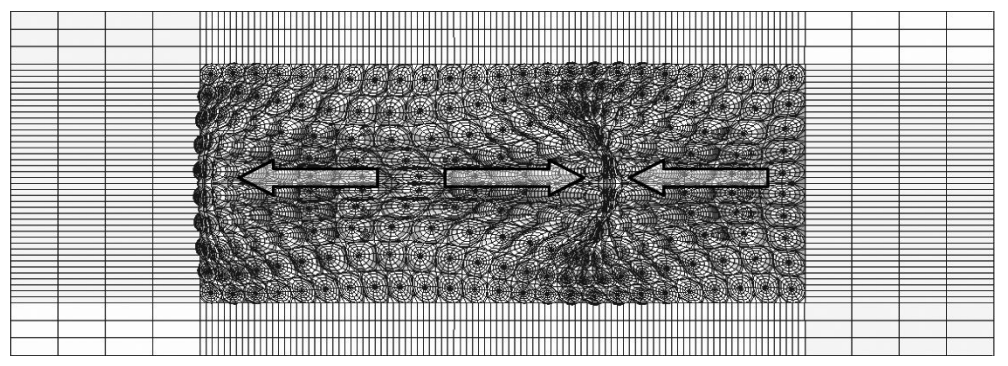

Fig. 50. EIDL 10th mode $-979 \mathrm{~Hz}$ - in plane triple opposite horizontal translation.

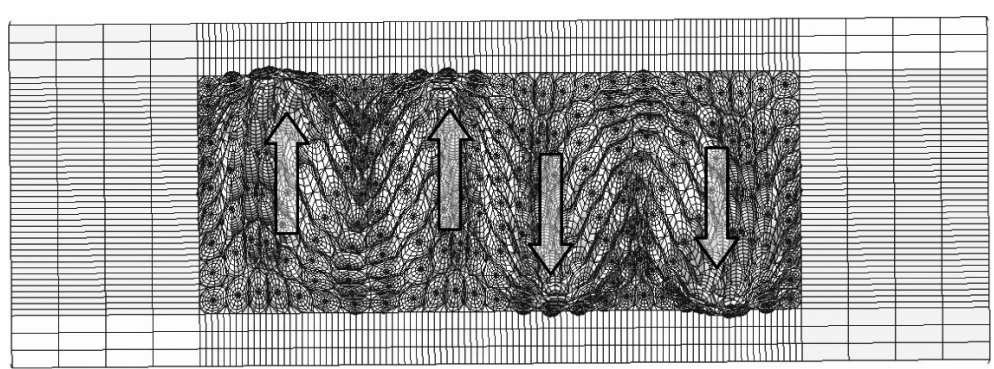

Fig. 51. EIDL 11th mode $-993 \mathrm{~Hz}$ - in plane quadruple opposite vertical translation.

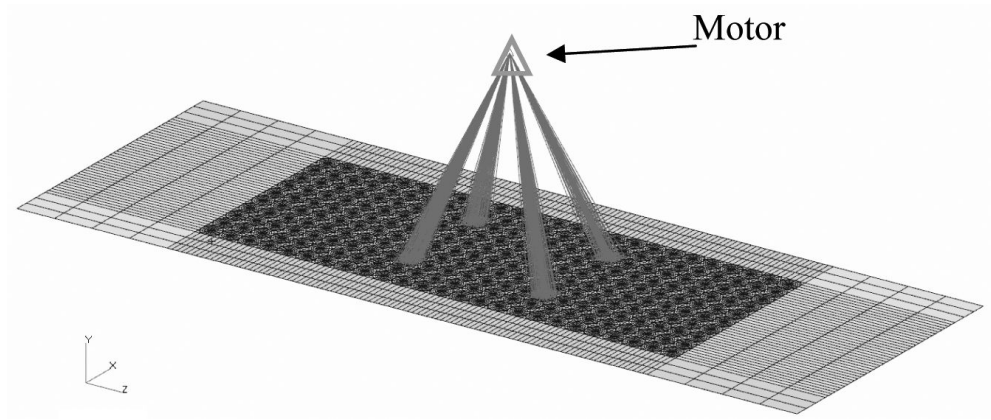

Fig. 52. Elasto-inertial dynamic layer attached to a plate with a vibrating motor.

excitation (upper sketch) the EIDL is planar due to zero displacements in the plate. On the other hand, the existence of dynamic excitation results in micro displacement along the plate such as the first bending mode (lower sketch). Since the upper region of the EIDL is submitted to larger displacement than the lower region of the EIDL, a lateral wave pressure is generated.

This wave pressure is illustrated in Fig. 54 by two opposite arrows representing the lateral displacement of the balls. This results in displacement of the balls in perpendicular to the structure mode direction, which is the essence of the vertical mode reshaping. At any given excitation frequency, different type of wave pressure and mode are generated. These wave pressures excite different normal modes of the EIDL that dynamically act in suppression of the displacement amplitude.

The concluding issue of this research would be to find how the size of the EIDL cells influences its performance. This issue is examined in the next section.

\subsection{The influence of cells size on the EIDL performance}

In order to better understand the sensitivity of the EIDL to the cell size of the multi-modal dynamic absorber, an additional couple of cases with different cell size are investigated. These cases are EIDL with a unit cell size of 


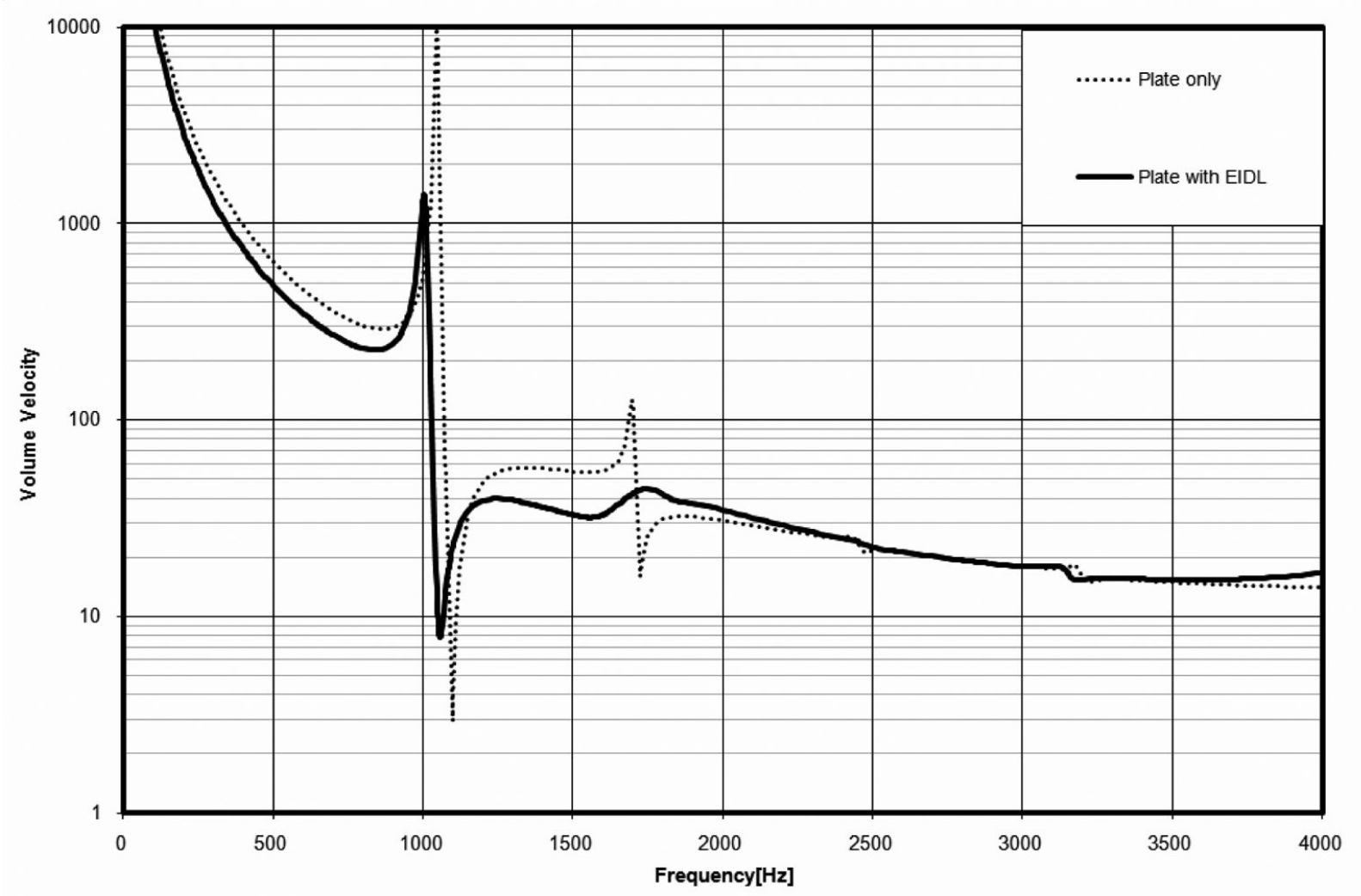

Fig. 53. Frequency response curves for two cases of free-free plate Plate without EIDL (dotted), Plate with added EIDL (solid).

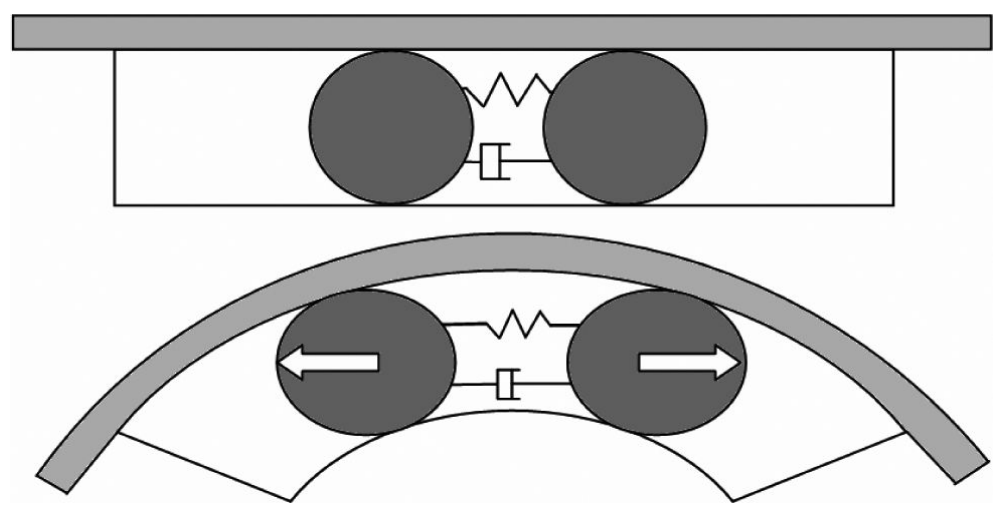

Fig. 54. Wave pressure generation in a EIDL.

$4 \mathrm{~mm}$ and $5 \mathrm{~mm}$, attached to the same plate used before. Due to the constraint of keeping the EIDL dimensions to a $32 \mathrm{~mm}$ by $80 \mathrm{~mm}$, the number of cells used in the $4 \mathrm{~mm}$ EIDL is 152 (8X19) and the number of cells used in the $5 \mathrm{~mm}$ EIDL is 90 (6X15). Figure 55 below demonstrates the dimension difference between the $3 \mathrm{~mm}$ EIDL and the $5 \mathrm{~mm}$ EIDL.

Furthermore, in order to understand the sensitivity of the EIDL to the general mass of the multi-modal dynamic absorber, each of the former cases is investigated with three different ball densities. For each of the EIDL cases, the metal balls density was alternated between Tungsten $-\rho_{\text {tung }}=19300\left[\mathrm{~kg} / \mathrm{mm}^{3}\right]$, Steel $\rho_{S t}=7800\left[\mathrm{~kg} / \mathrm{mm}^{3}\right]$ and Aluminium $-\rho_{A l}=2700\left[\mathrm{~kg} / \mathrm{mm}^{3}\right]$. 

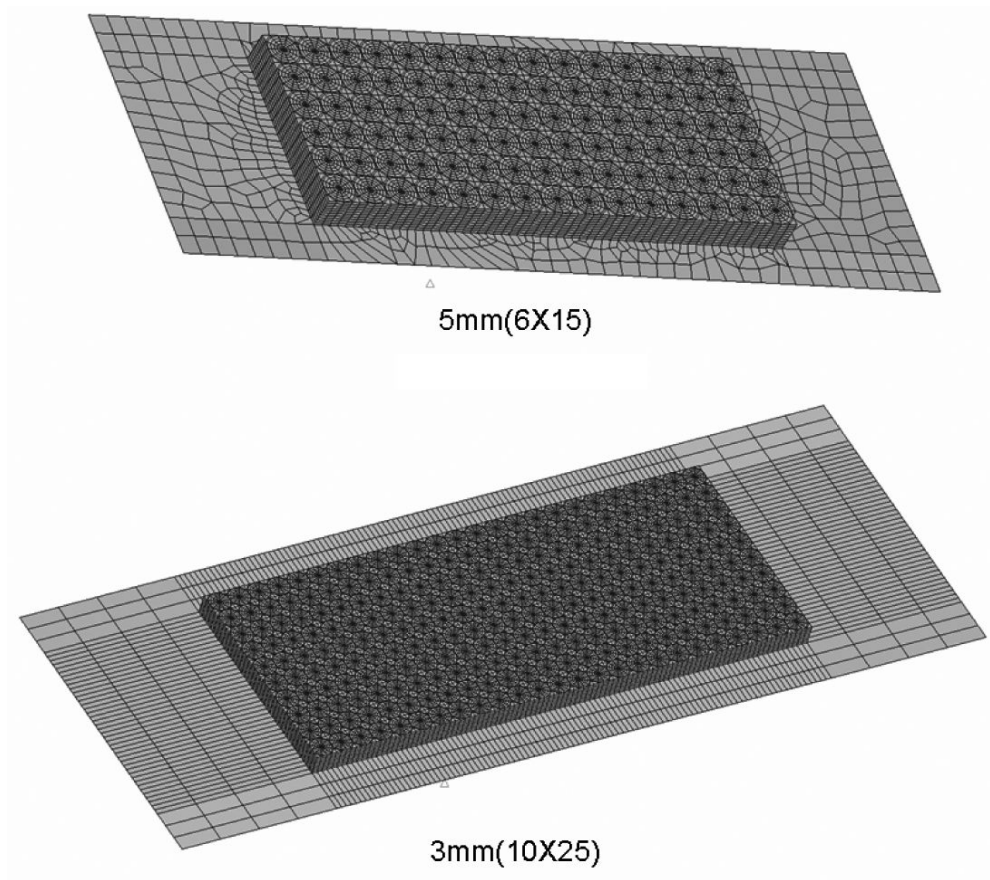

Fig. 55. Two cases of a Plate with added EIDL, up $-5 \mathrm{~mm}$ EIDL, bottom $-3 \mathrm{~mm}$ EIDL.

Alternating both the cell size and the metal ball densities has generated nine cases of EIDL. For each of these cases, a frequency response simulation was conducted, where the motor is subjected to a sinusoidal excitation as in the previous case. The results for these frequency response simulations are given in the Figs 56 to 58.

Figure 56 is essentially identical to Figure 53 except for the added cases of the tungsten and aluminium. It can be seen that the tungsten case demonstrates better performance at the lower frequency range and the aluminium case is better at the higher frequency range. Taken as a whole, the preferred option for the $3 \mathrm{~mm}$ EIDL would be the steel ball due to its global attenuation performance over the whole frequency range.

The response manner of the $4 \mathrm{~mm}$ EIDL curves is quite similar for the $3 \mathrm{~mm}$ EIDL in a qualitative point of view. Figure 57 shows again that the tungsten case demonstrates better performance at the lower frequency range and the Aluminium case is better at the higher frequency range. Yet again, the preferred option for the 4 mm EIDL would be the steel ball due to its global attenuation performance over the whole frequency range.

In the case of using a $5 \mathrm{~mm}$ EIDL it can be seen from Fig. 58 that the better vibration attenuation performance is achieved through the aluminium ball EIDL case. The aluminium demonstrates a predominance performance over the whole frequency range.

\subsection{Concluding remarks}

This section reviews the direct continuation from the development of the multi-modal dynamic absorber, in to its expansion to the conceptual model of the EIDL. This is done in order to achieve a conceptual model that has a global, wide frequency range and effective dynamic absorption of vibration in electro-optical systems. The EIDL has proven to be effective in a wide frequency range excitation and with a negligible volume and weight penalty pay. Thanks to the simple design of the EIDL, it allows a simple attachment to any optical system.

The most recent attempt to control the sound transmission in aircraft and automotive systems using a combination of viscoelastic material and mass inclusion was published by Idrisi et al. (Mercedes Technology Center 2010). This research used detailed analysis of the behaviour of the mass inclusions, highlighting controlled stiffness variation of the mass-spring-damper systems inside the heterogeneous blanket. Idrisi et al. missed out the basic benefit of the structure by using a 1D spring damper system to characterize the enclosure dynamic behaviour. By doing that they essentiality discarded the 3D behaviour of the system and thus the potential of wide range vibration attenuation. 


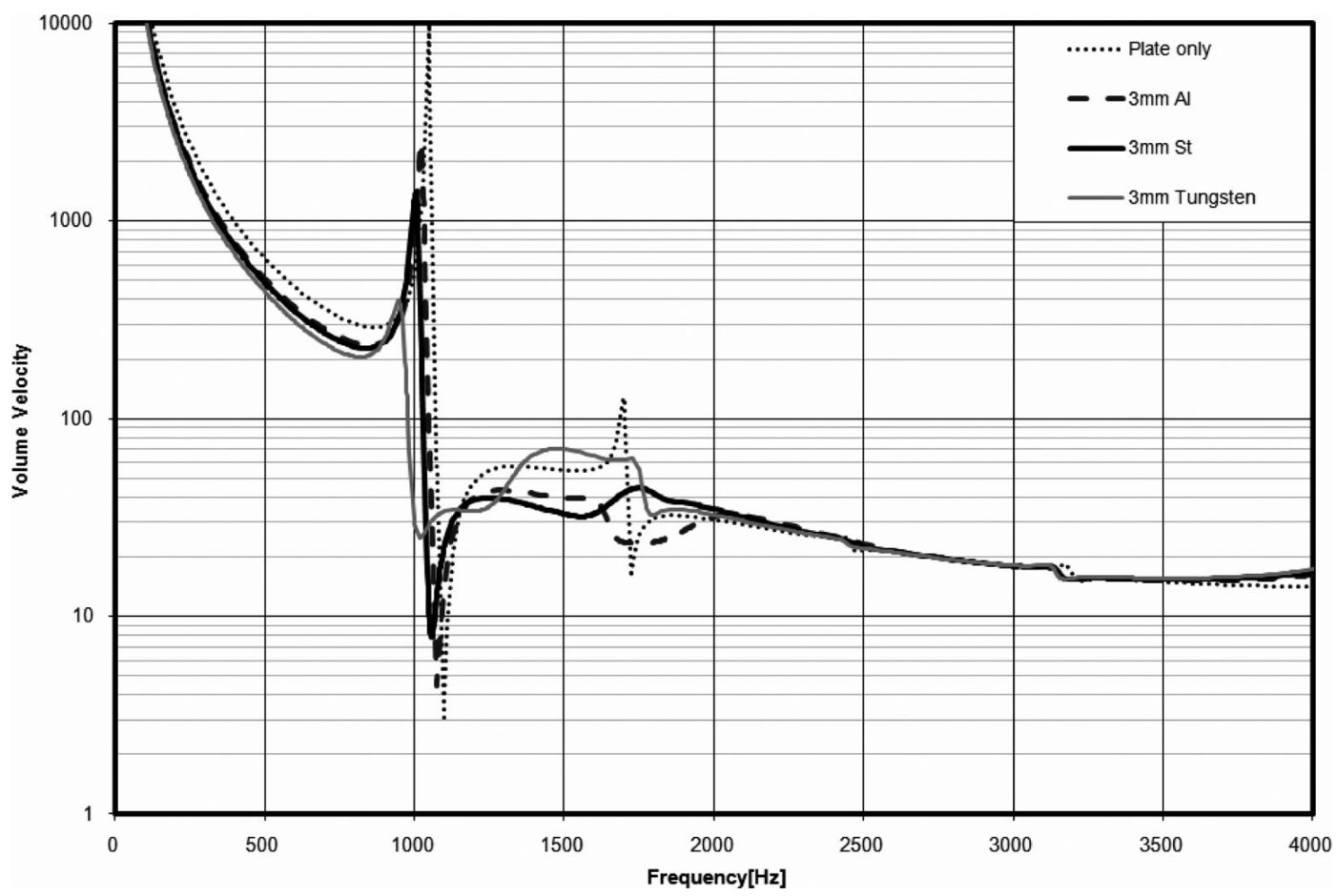

Fig. 56. Frequency response curves for four cases of free-free plate Plate only (dotted), Plate with added Steel ball EIDL (solid black), Plate with added Aluminium ball EIDL (dashed), Plate with added Tungsten ball EIDL (solid grey).

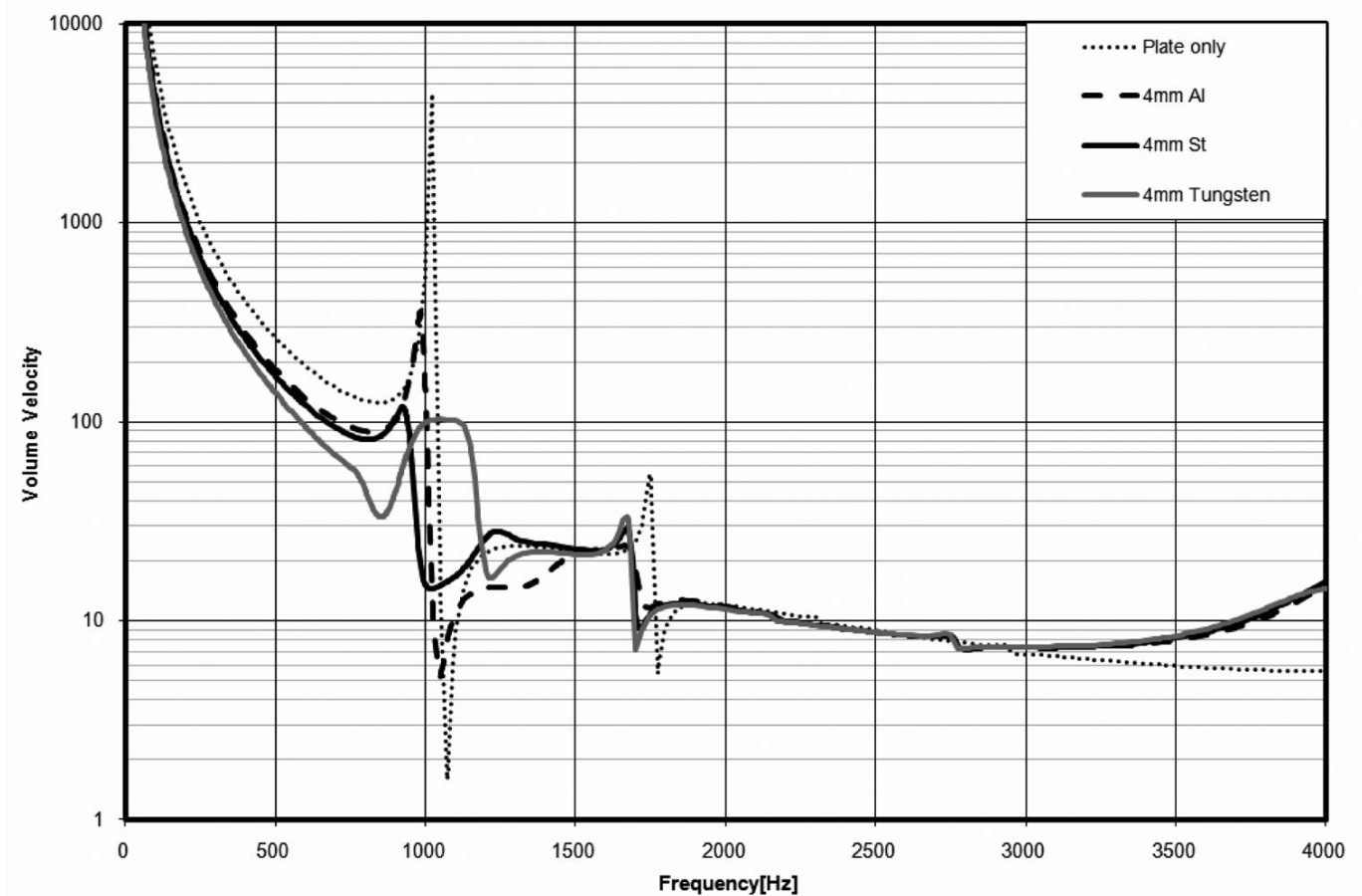

Fig. 57. Frequency response curves for four cases of free-free plate Plate only (dotted), Plate with added Steel ball EIDL (solid black), Plate with added Aluminium ball EIDL (dashed), Plate with added Tungsten ball EIDL (solid grey). 


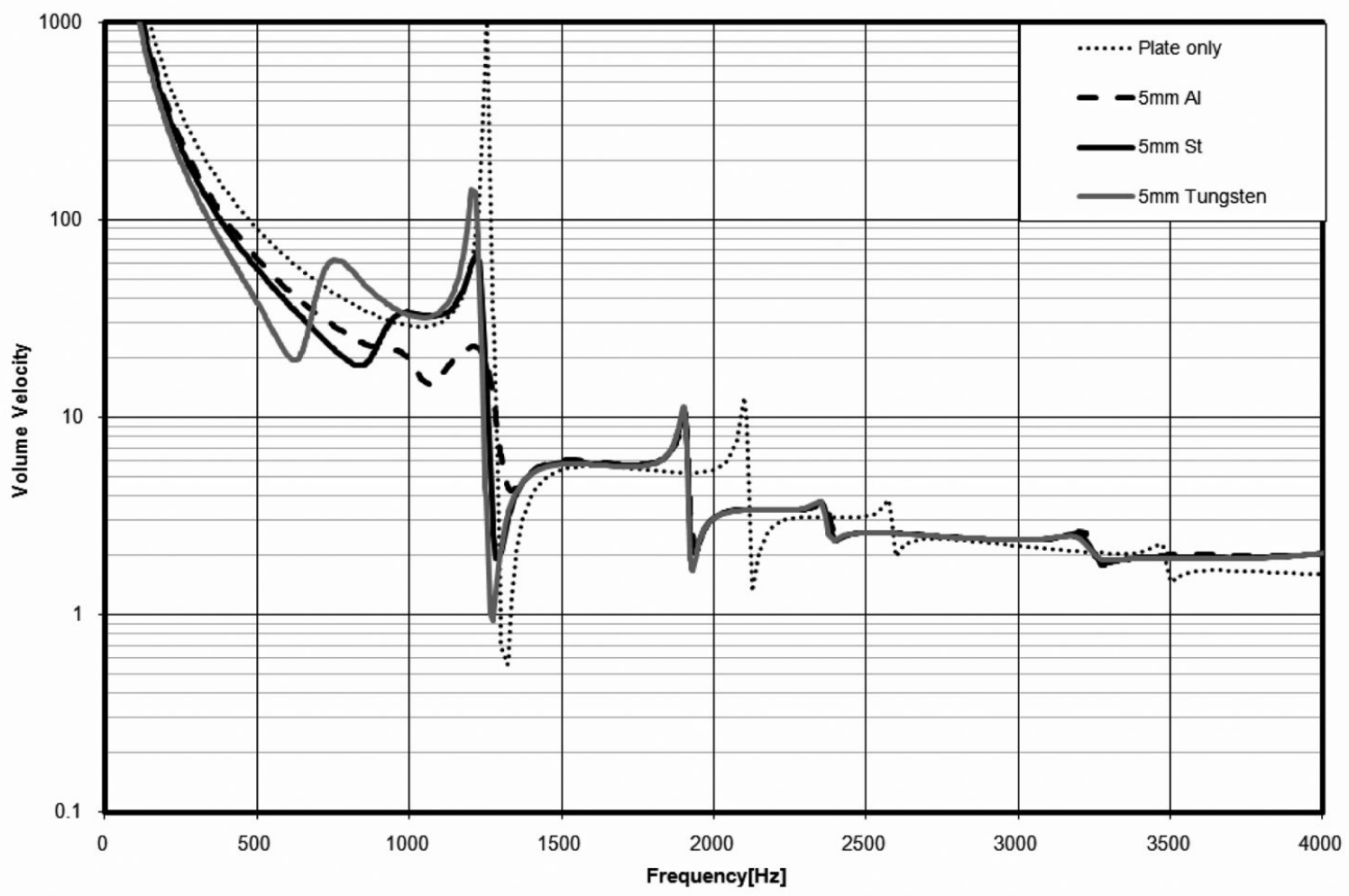

Fig. 58. Frequency response curves for four cases of free-free plate Plate only (dotted), Plate with added Steel ball EIDL (solid black), Plate with added Aluminium ball EIDL (dashed), Plate with added Tungsten ball EIDL (solid grey).

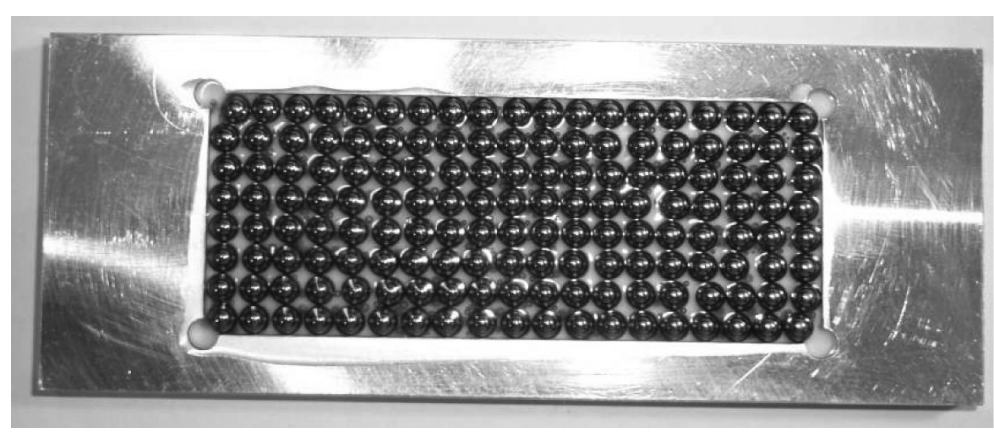

Fig. 59. EIDL Embedded in an Aluminium plate.

The next task of this work would be to test the aforementioned model in a full scale mechanical system to prove its substantial ability of vibration attenuation.

\section{Experimental validation of the elasto-inertial dynamic layer}

As described in Section 4, the elasto-inertial dynamic layer (EIDL) is essentially an expended MMDA. Section 4 reviews the direct continuation from the development of the multi-modal dynamic absorber, in to its expansion to the conceptual model of the EIDL. The EIDL is constructed through a large number of metal balls embedded in a viscoelastic matrix as shown in Fig. 59. A detailed simulation data showed evidence that this conceptual model has an overall, wide frequency range and effective dynamic absorption of vibration of mechanical systems. The EIDL 
Table 1

EIDL specimen's properties

\begin{tabular}{|c|c|c|c|c|c|c|c|c|}
\hline Specimen property & $3 \mathrm{~K}^{*}$ & $3 X$ & $4 \mathrm{~K}$ & $4 X$ & $5 \mathrm{~K}$ & $5 X$ & Combined & Si-only \\
\hline $\begin{array}{l}\text { Ball Diameter } \\
\text { Number of Balls } \\
25 \times 10\end{array}$ & $\begin{array}{c}3 \mathrm{~mm} \\
250 \\
26 \mathrm{X} 12\end{array}$ & $\begin{array}{c}3 \mathrm{~mm} \\
312 \\
19 \mathrm{X} 8\end{array}$ & $\begin{array}{c}4 \mathrm{~mm} \\
152 \\
19 \times 5,20 \times 4\end{array}$ & $\begin{array}{c}4 \mathrm{~mm} \\
175 \\
15 \mathrm{X} 6\end{array}$ & $\begin{array}{c}5 \mathrm{~mm} \\
90 \\
15 \times 4,16 \times 3\end{array}$ & $\begin{array}{c}5 \mathrm{~mm} \\
108\end{array}$ & $\begin{array}{l}3 \mathrm{~mm}-96 \\
4 \mathrm{~mm}-54 \\
5 \mathrm{~mm}-30 \\
\end{array}$ & $\begin{array}{l}\text { None } \\
\text { None }\end{array}$ \\
\hline EIDL Mass & $31 \mathrm{gr}$ & $36 \mathrm{gr}$ & $42 \mathrm{gr}$ & $48 \mathrm{gr}$ & $50 \mathrm{gr}$ & $58 \mathrm{gr}$ & $46 \mathrm{gr}$ & $8 \mathrm{gr}$ \\
\hline
\end{tabular}

${ }^{*}$ Square $=\mathrm{K}$, Packed $=\mathrm{X}$

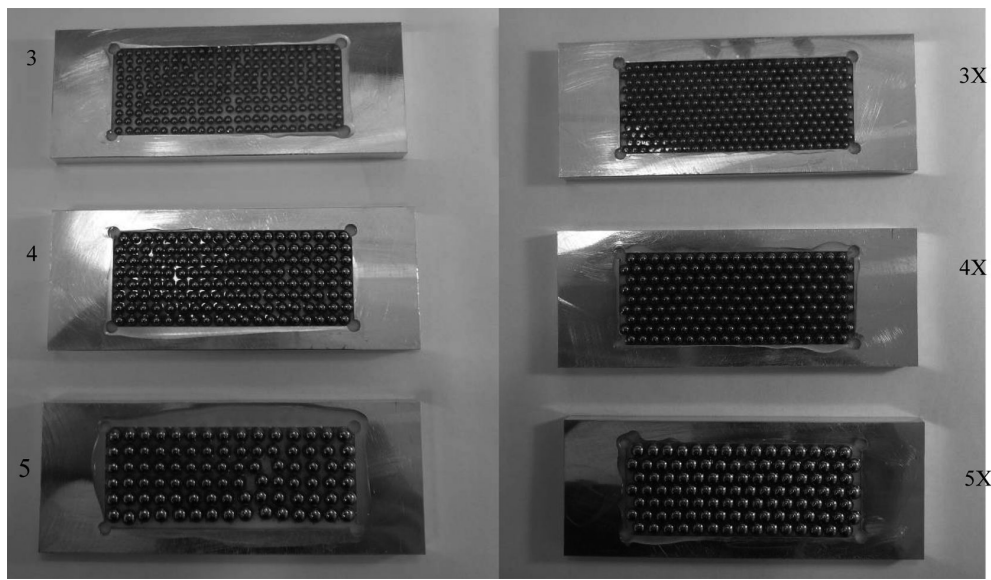

Fig. 60. EIDL Specimens.

has proven to be effective in a wide frequency range excitation and with a negligible volume and weight penalty pay. This section is dedicated to quantify the promising attenuation capacity of the EIDL.

\subsection{Plate with an embedded elasto-inertial dynamic layer-Manufactured specimens}

As described by the FE models, the plate used in the experiments is a $48 \mathrm{~mm}$ by $123 \mathrm{~mm}$ with a $7 \mathrm{~mm}$ thickness and made of aluminium. This plate has a centred machined void of $3 \mathrm{~mm}$ depth and in $32 \mathrm{~mm}$ by $80 \mathrm{~mm}$ dimensions. The EIDL was constructed according to the following steps. First, the exact number of steel balls was placed in to the void. Then silicon had been injected into the void to fill the gap between the balls. The following stage was the organization of the balls in rows and columns using a slender pin. This stage had to be carried out as accurate as possible in order to achieve the same ball relation used in the FE models. Finally, placing the plate in a $200^{\circ} \mathrm{C}$ preheated oven for two hours for the silicon to cure.

A total of nine different specimens with different EIDL configuration had been manufactured using this technique. In addition, a void without EIDL had been used as a reference. Table 1 reviews the different specimen's properties.

Note that the specimens marked as Square (K) are specimens that match the FE models (see Fig. 61). A specimen that contains the maximum balls possible (see Fig. 61) are marked as Packed (X). Furthermore, a 'combined' configuration of EIDL that inhabit the three different ball diameter are marked as Combined. Finally, a specimen with a void filled with silicon only is marked as SI-only.

\subsection{Plate with an embedded elasto-inertial dynamic layer - Frequency response curves}

The experimental rig used to measure the plates with EIDL transfer function, is illustrated in Fig. 63 accompanied by an actual photo (Fig. 62). The plate (3), loaded with a 710gr mass (4) that simulates the mass of the motor, is rested on ground (1) through a flexible packing foam block (2). An impact hammer (6) is used to induce vibration to the beam. A Brüel \& Kjær accelerometer (5) and a Data Physics Quattro (7) analyzer are used to measure the 


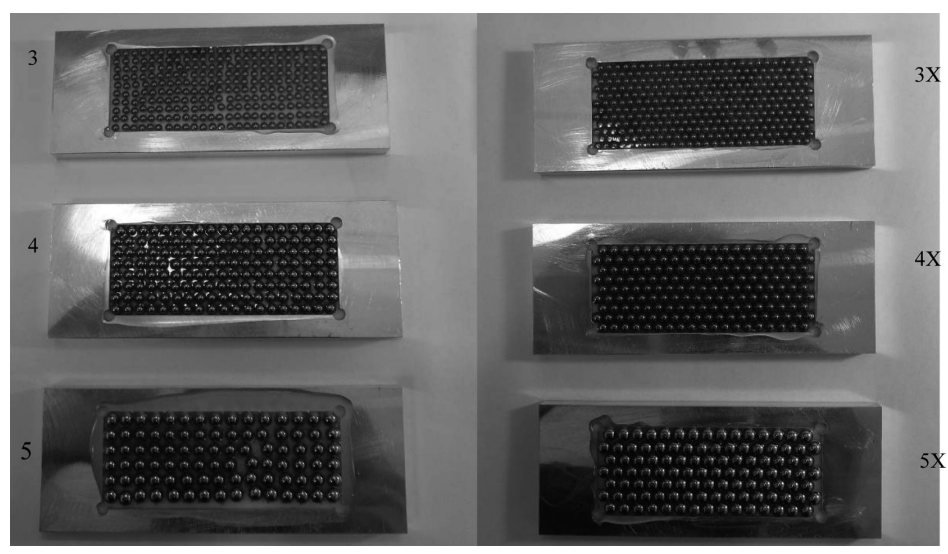

Fig. 61. EIDL Specimens.

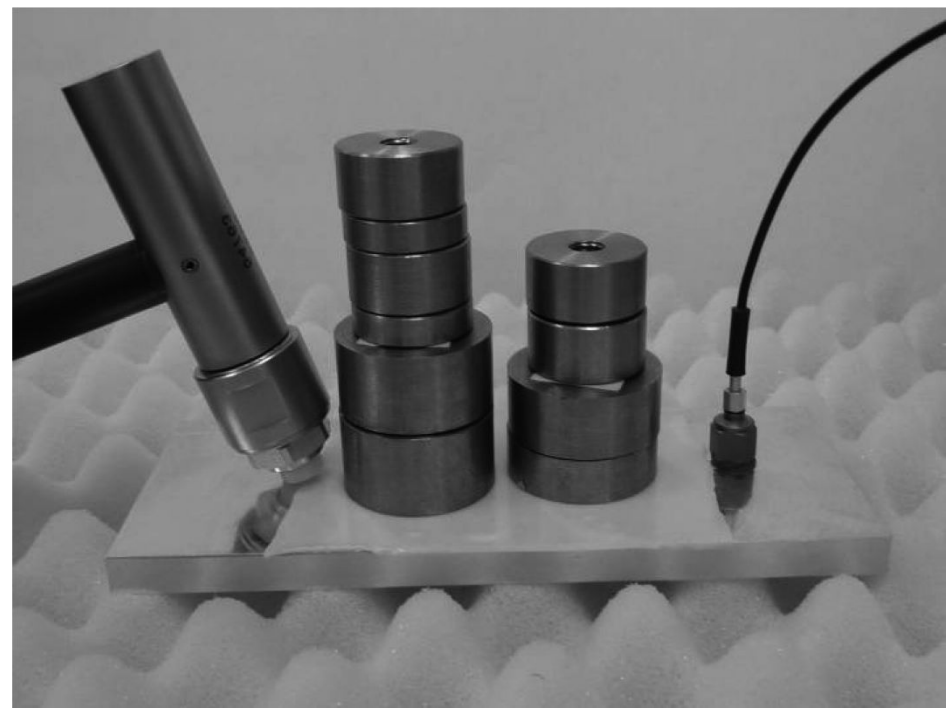

Fig. 62. Aluminium plate with EIDL loaded with 710 gr mass.

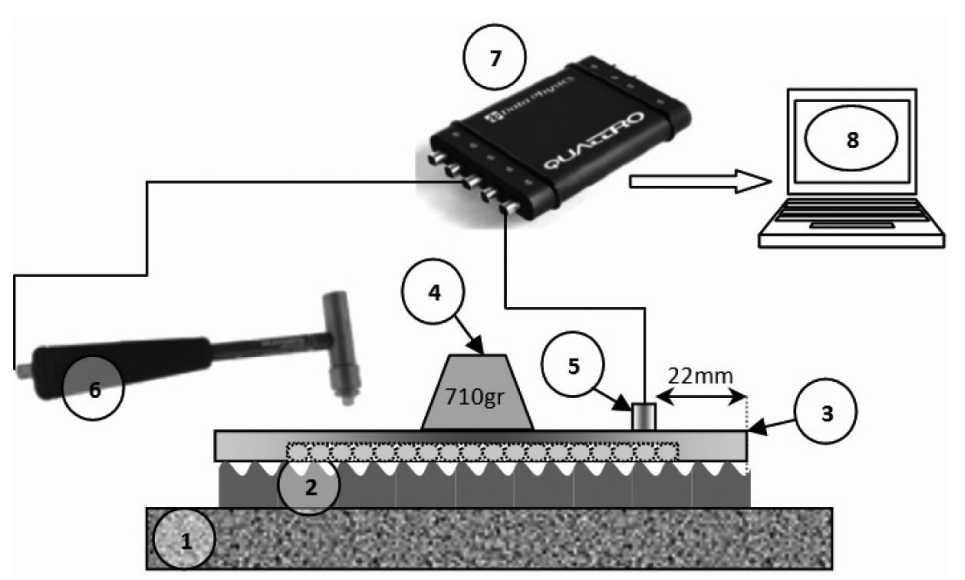

Fig. 63. Experimental rig diagram. 


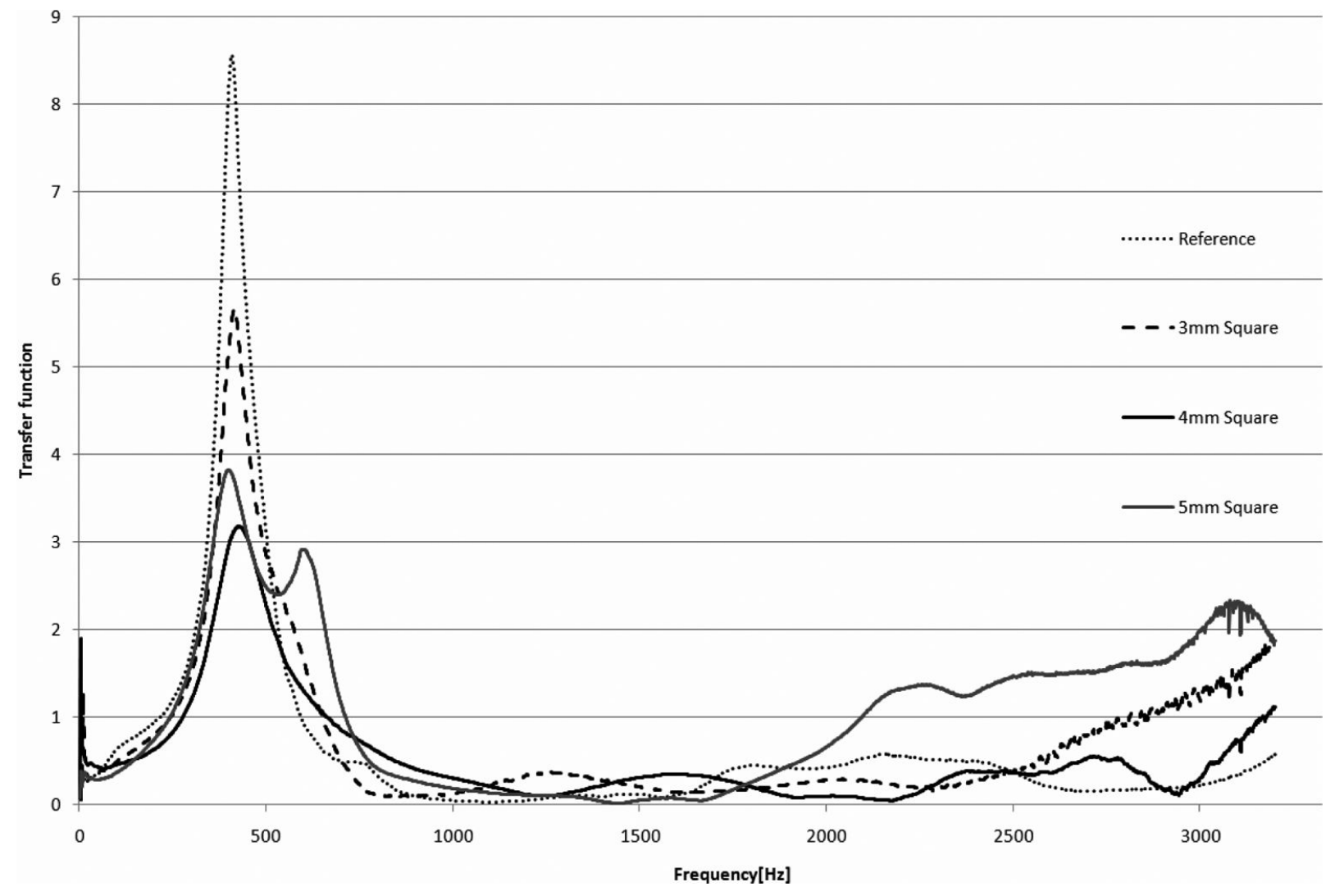

Fig. 64. EIDL Transfer function curves - Square Vs. Reference.

vibration and the force induced by the impact hammer. The vibration data from the analyzer is recorded by laptop (8) and a transfer function curve is produced using Signalcalc software.

The transfer function curves measured are given in Figs 64 and 65. These figures also include the measured curve for the case of an aluminium plate without EIDL as reference.

It can be seen from Figs 64 and 65 that the best absorption capacity is reached in the case with a 4 mm diameter ball size. This was also inferred by the simulation on previous section. That goes for both the case of 'Square' and 'Packed' configurations. Figure 66 shows the difference between the two best cases, ie $4 \mathrm{~mm}$ Packed and $4 \mathrm{~mm}$ Square.

It becomes visible from Fig. 66 that the $4 \mathrm{~mm}$ Square case has a better attenuation capacity over the higher frequency range and a worse attenuation capacity at the $500 \mathrm{~Hz}$ peak region. It is important to stress that the results are correct to the point of measurement, i.e. $22 \mathrm{~mm}$ from the plate edge. In order to get an enhanced prospective of the EIDL performance, a Microphonics measurement is taken for each of the cases at the next section.

\subsection{Plate with an embedded elasto-inertial dynamic layer - Microphonics}

The most consistent way to assess the EIDL noise reduction capacity is through using a microphone to measure its true noise radiation. Using a Brüel \& Kjær type 2669 microphone (Fig. 67) allows an accurate noise radiation measurement of the whole plate surfaces. As oppose to earlier measurements, which gave a general impression over a limited region of the specimen, using a microphone provides an insight of the EIDL capacity at the system level as a whole.

The experimental rig used to measure the plates noise radiation is illustrated in Fig. 69, accompanied by an actual photo (Fig. 68). The plate (3) is rested on ground (1) through a flexible packing foam block (2). An impact hammer (4) is used to induce vibration to the plate. A Brüel \& Kjær type 2669 microphone (5) with a power supply (6) 


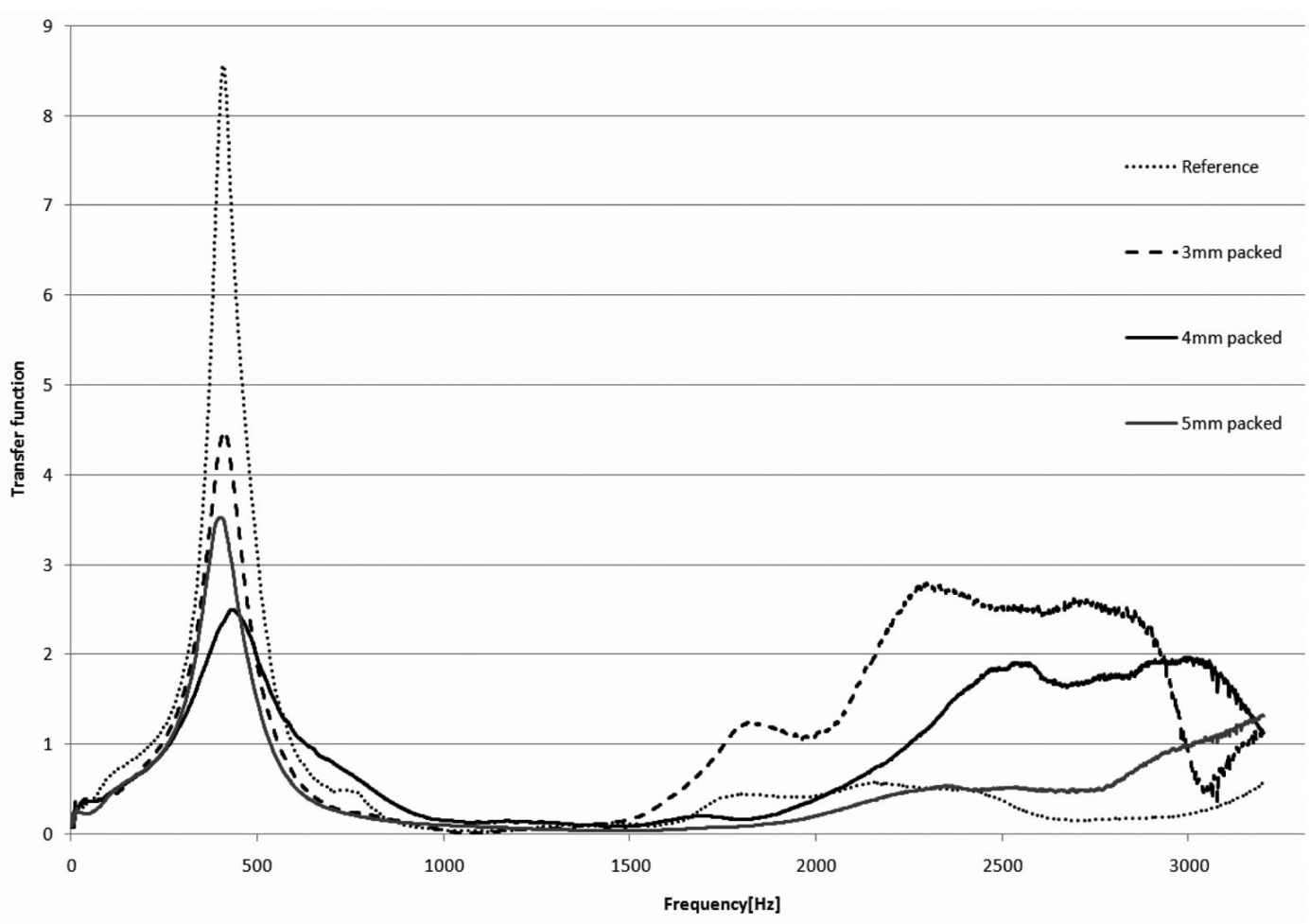

Fig. 65. EIDL Transfer function curves - Packed vs. Reference.

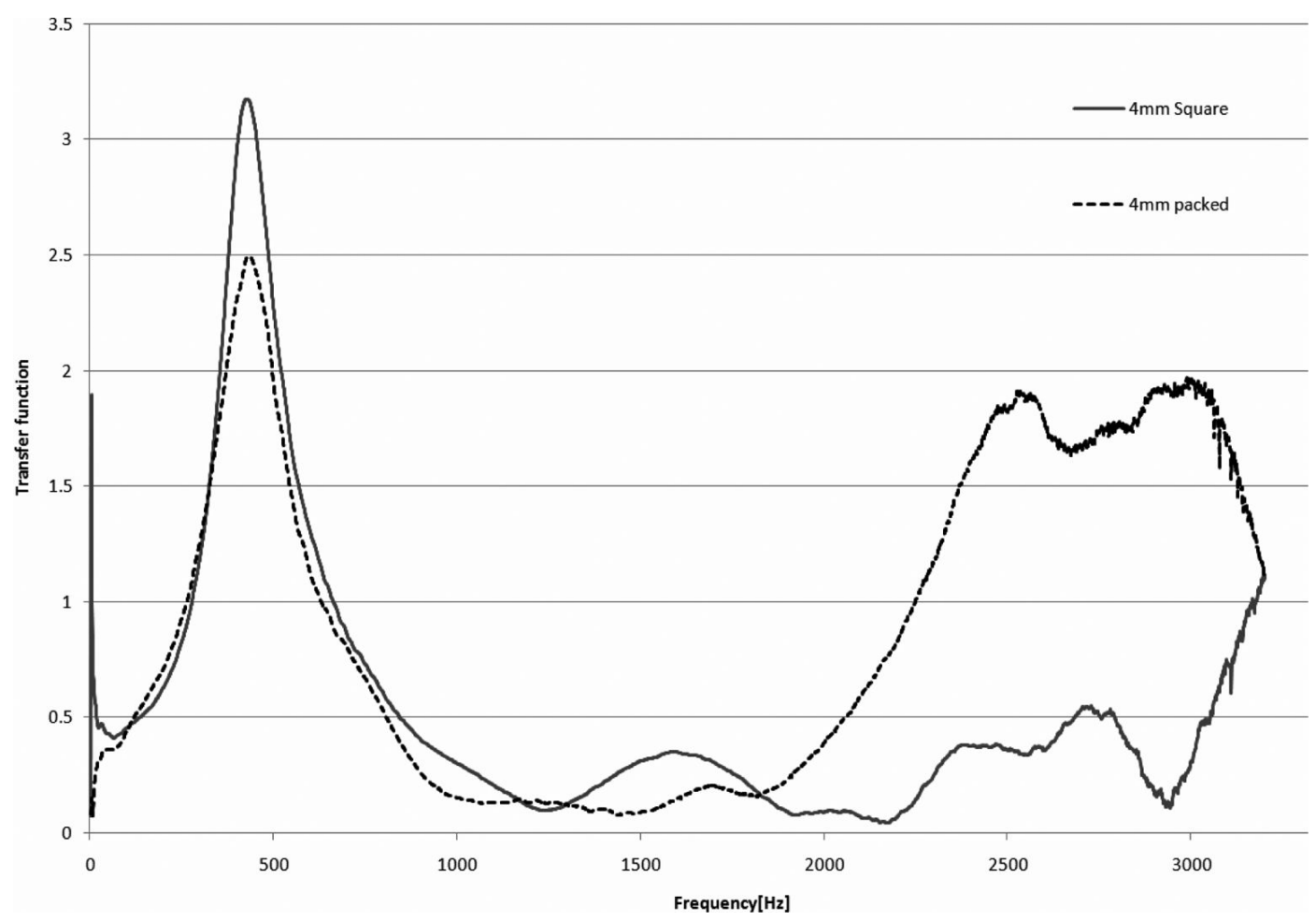

Fig. 66. EIDL Transmissibility curves -4 mm Packed vs. 4 mm Square. 

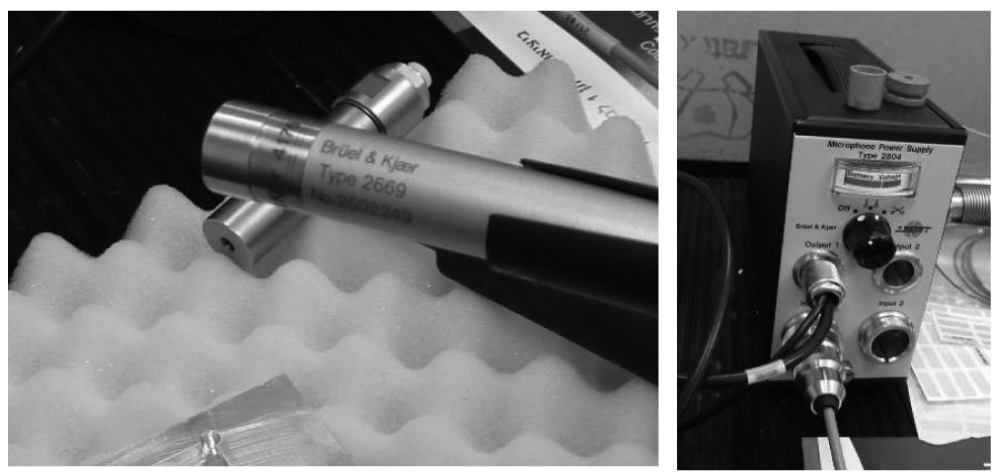

Fig. 67. Brüel \& Kjær type 2669 Microphone and power supply unit.

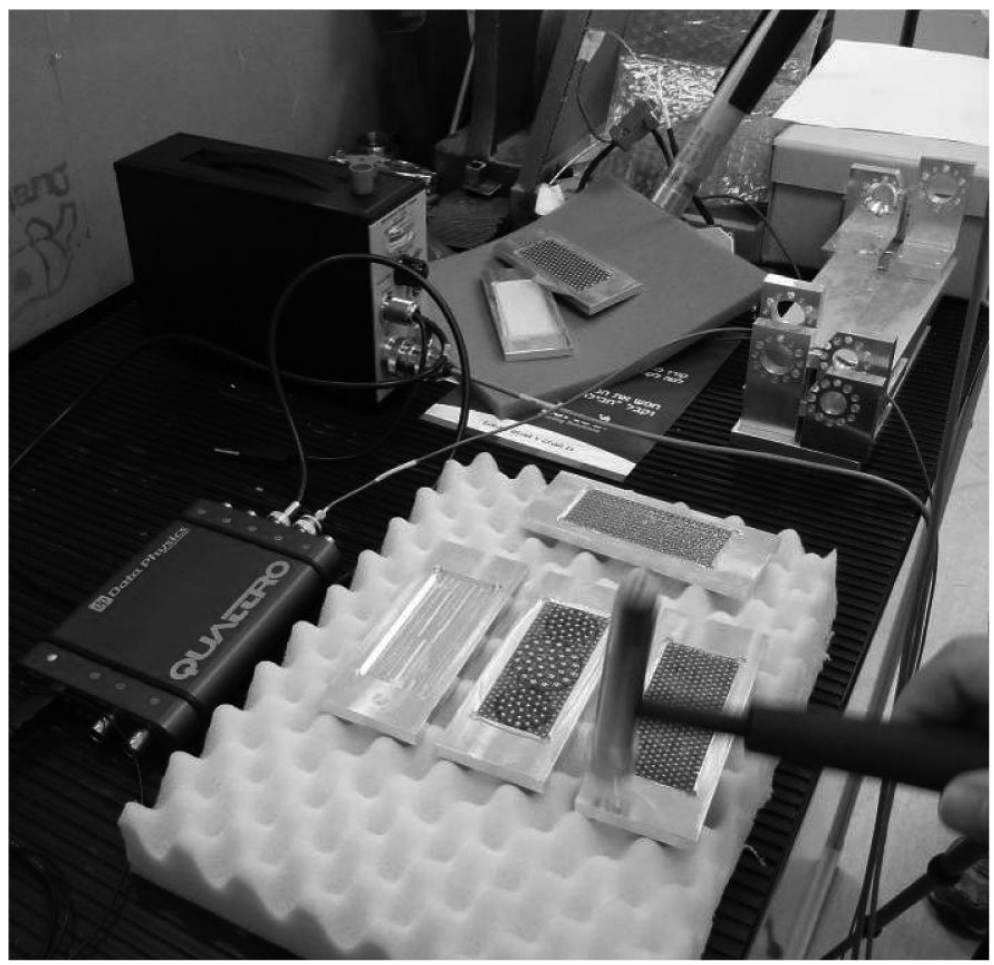

Fig. 68. Microphonics Experimental rig Photo.

connected to a Data Physics Quattro (7) analyzer, are used to measure the vibration and the force induced by the impact hammer. The vibration data from the analyzer is recorded by a laptop (8) and a transfer function curve is produced using Signalcalc software.

Figures 70 to 71 show the acoustic radiation measurements taken for each of the EIDL configurations given in table 1. As seen in Fig. 70, the reference measurement comprised of many sharp narrow peaks, is an evidence of a wide range noise radiation. Those peaks are dramatically reduced by each of the Square EIDL configurations.

Furthermore, it can be seen that the specimen Si-Only has negligible noise reduction and serves no purpose in vibration attenuation.

An even more dramatic peak cut off can be seen in Fig. 71 where the 'Combined' EIDL configuration flattens the whole noise radiation profile to a staggering $1 \%$ of the noise levels, over an impressive $20 \mathrm{kHz}$ frequency range. Figure 72 show a comparison between the $4 \mathrm{~mm}$ EIDL configuration to the 'Combined' one. It can be seen that the 


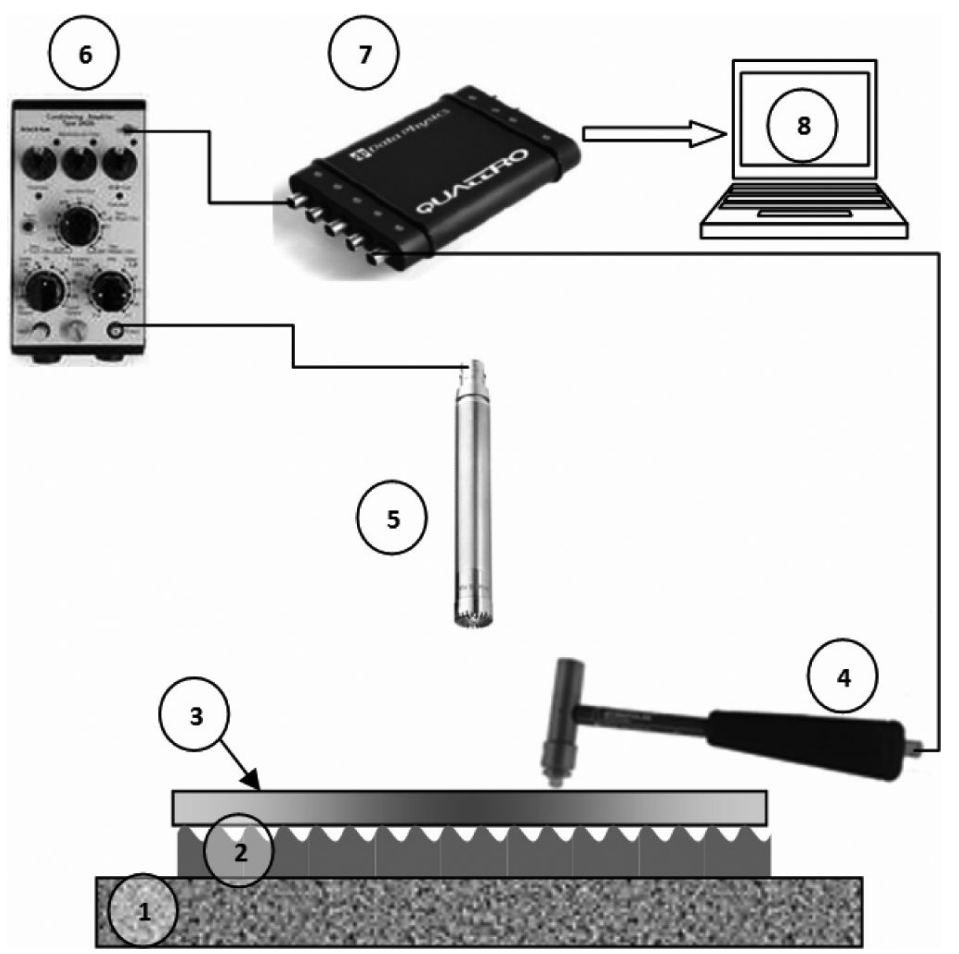

Fig. 69. Microphonics Experimental rig diagram.

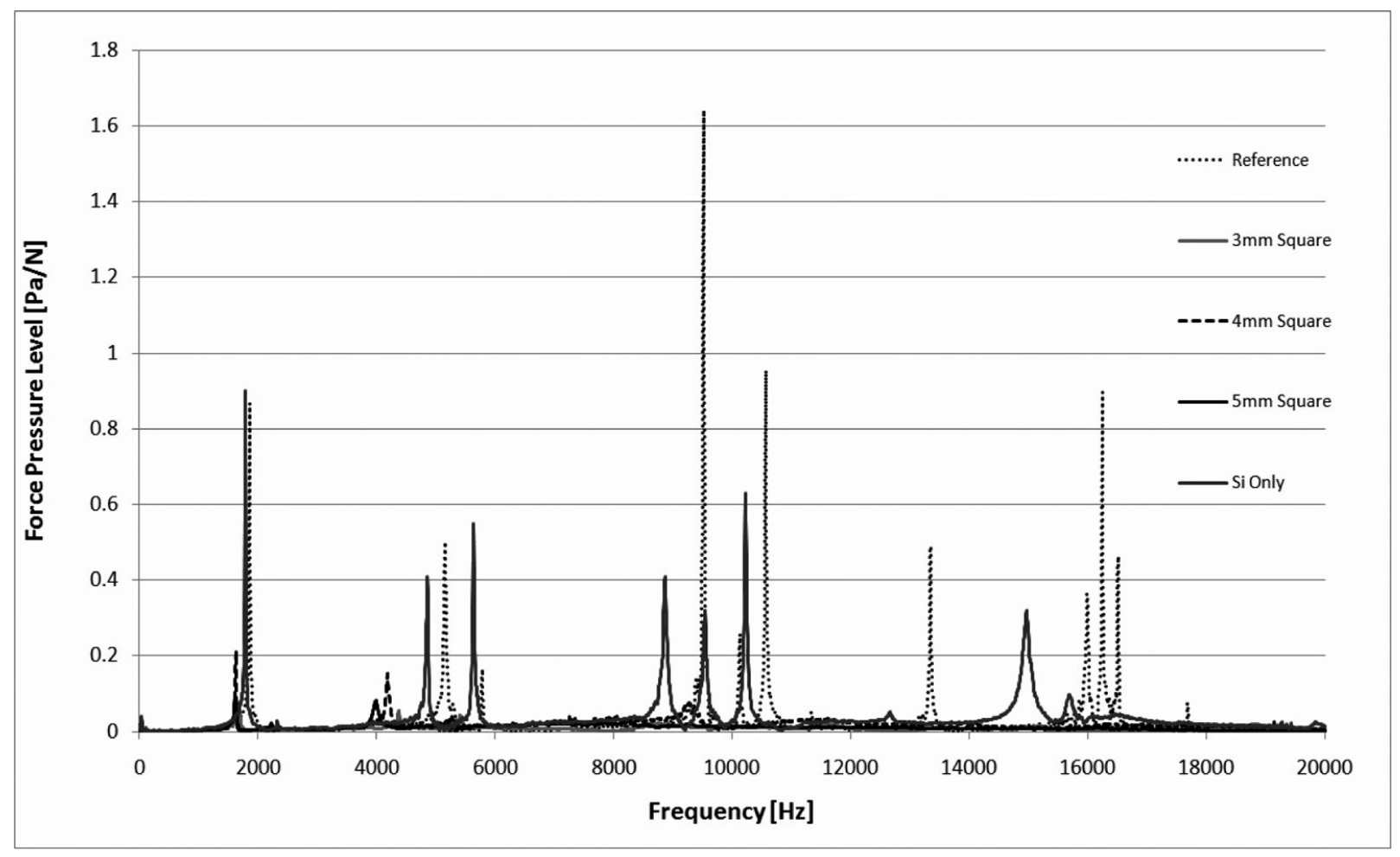

Fig. 70. Microphonics measurements for Square EIDL configurations. 


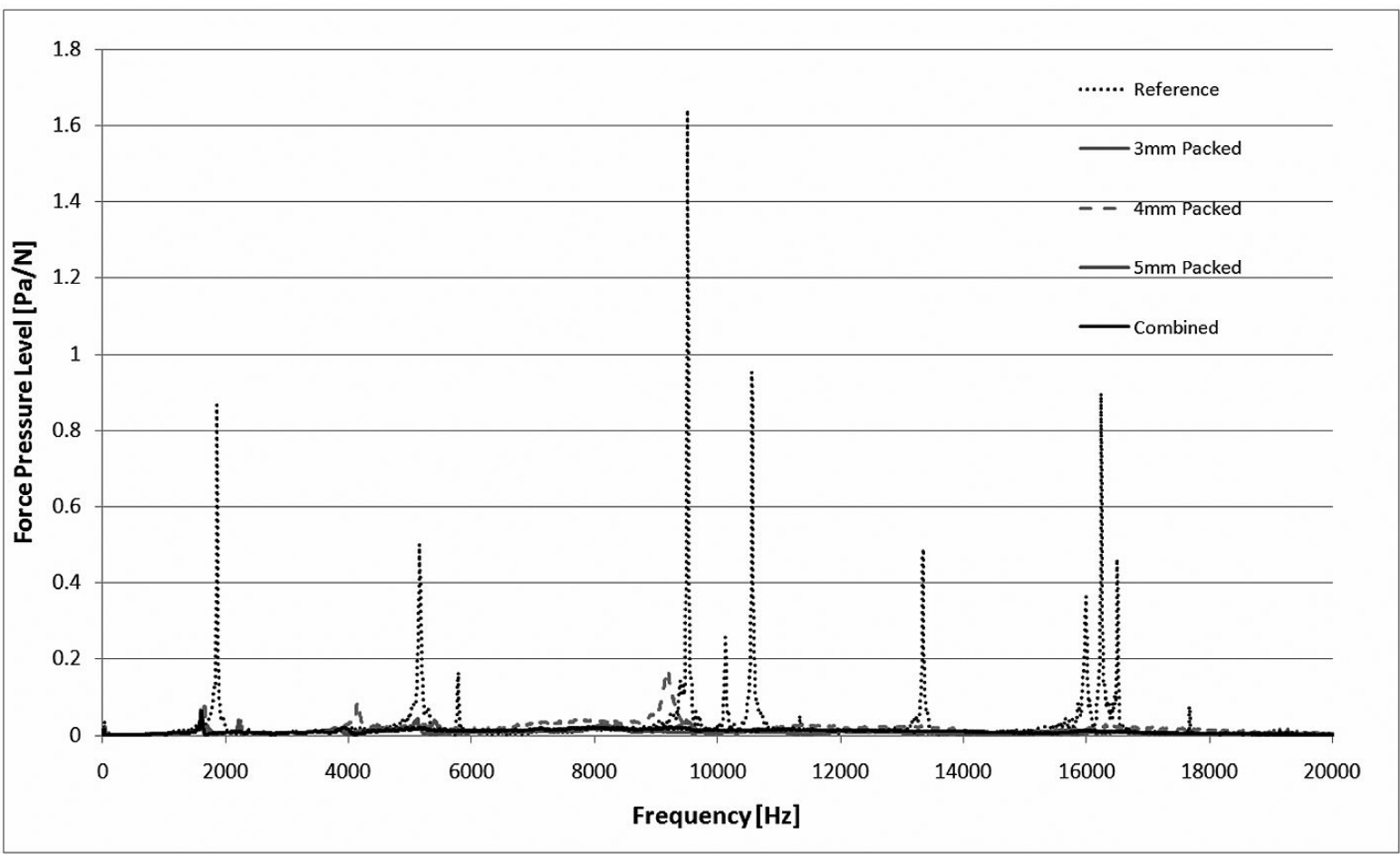

(a)

Fig. 71. (a) Microphonics measurements for Packed EIDL configurations

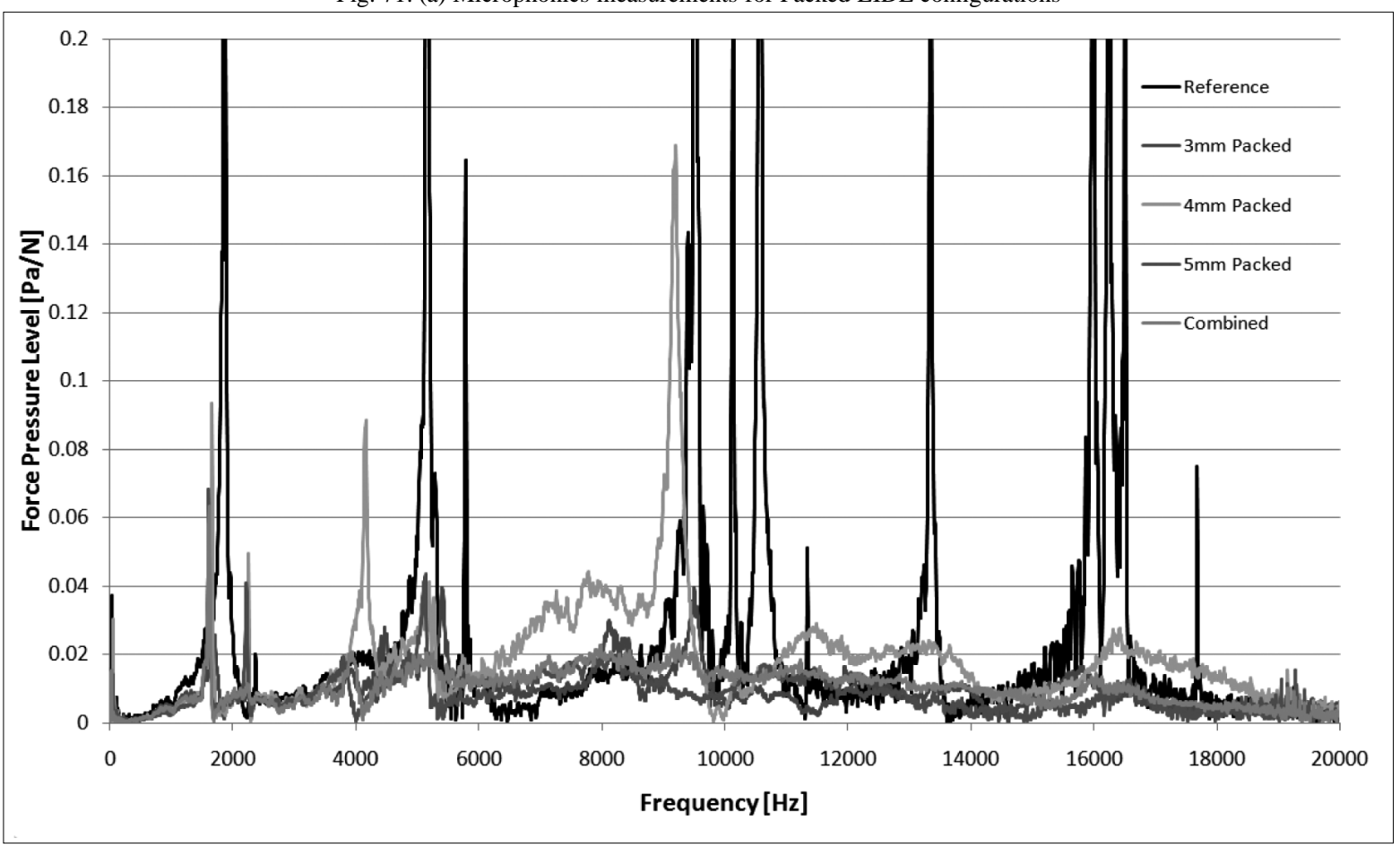

(b)

Fig. 71. (b) Microphonics measurements for Packed EIDL configurations- zoom view. 


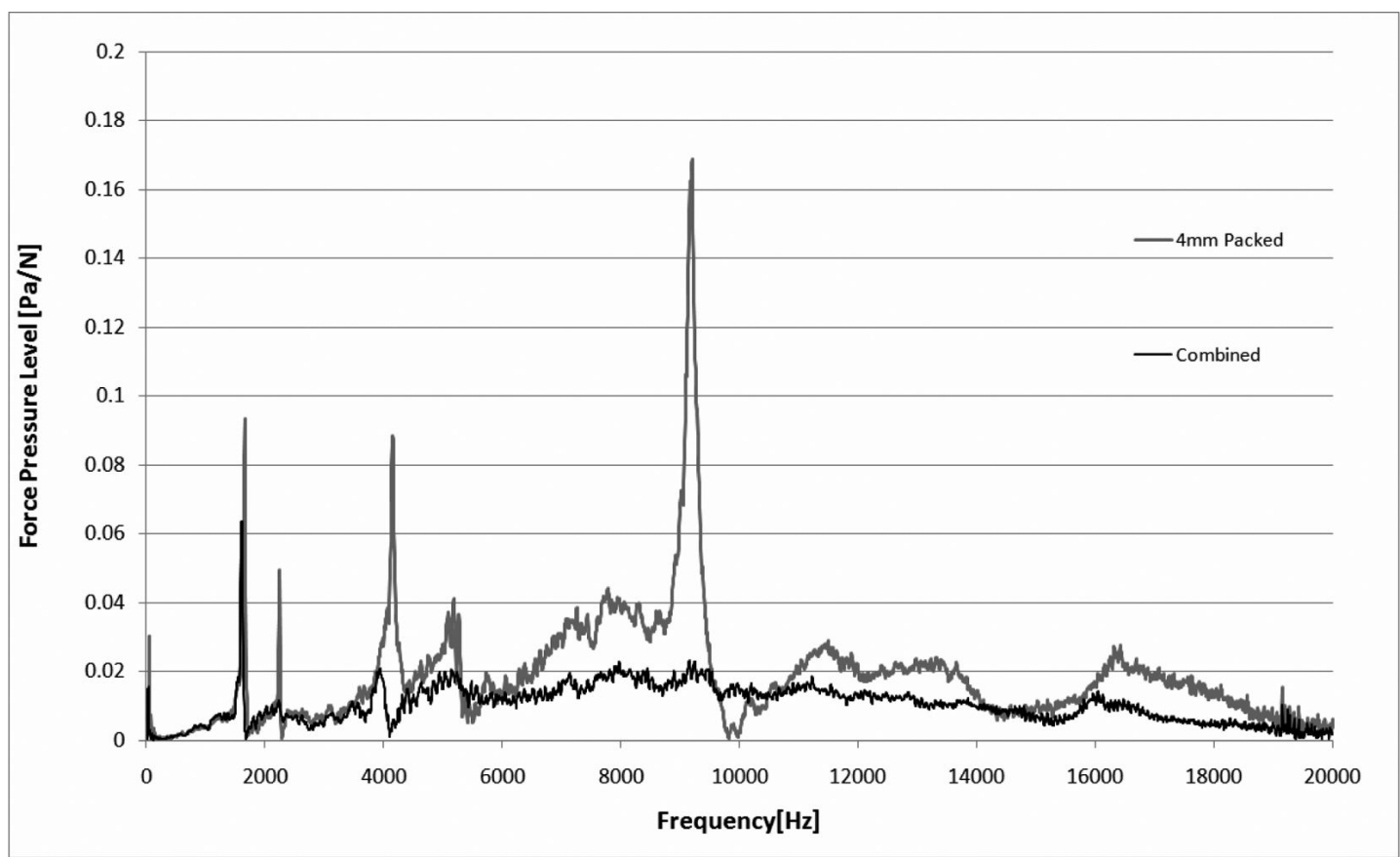

Fig. 72. $4 \mathrm{~mm}$ Packed Vs. Combined EIDL configuration.

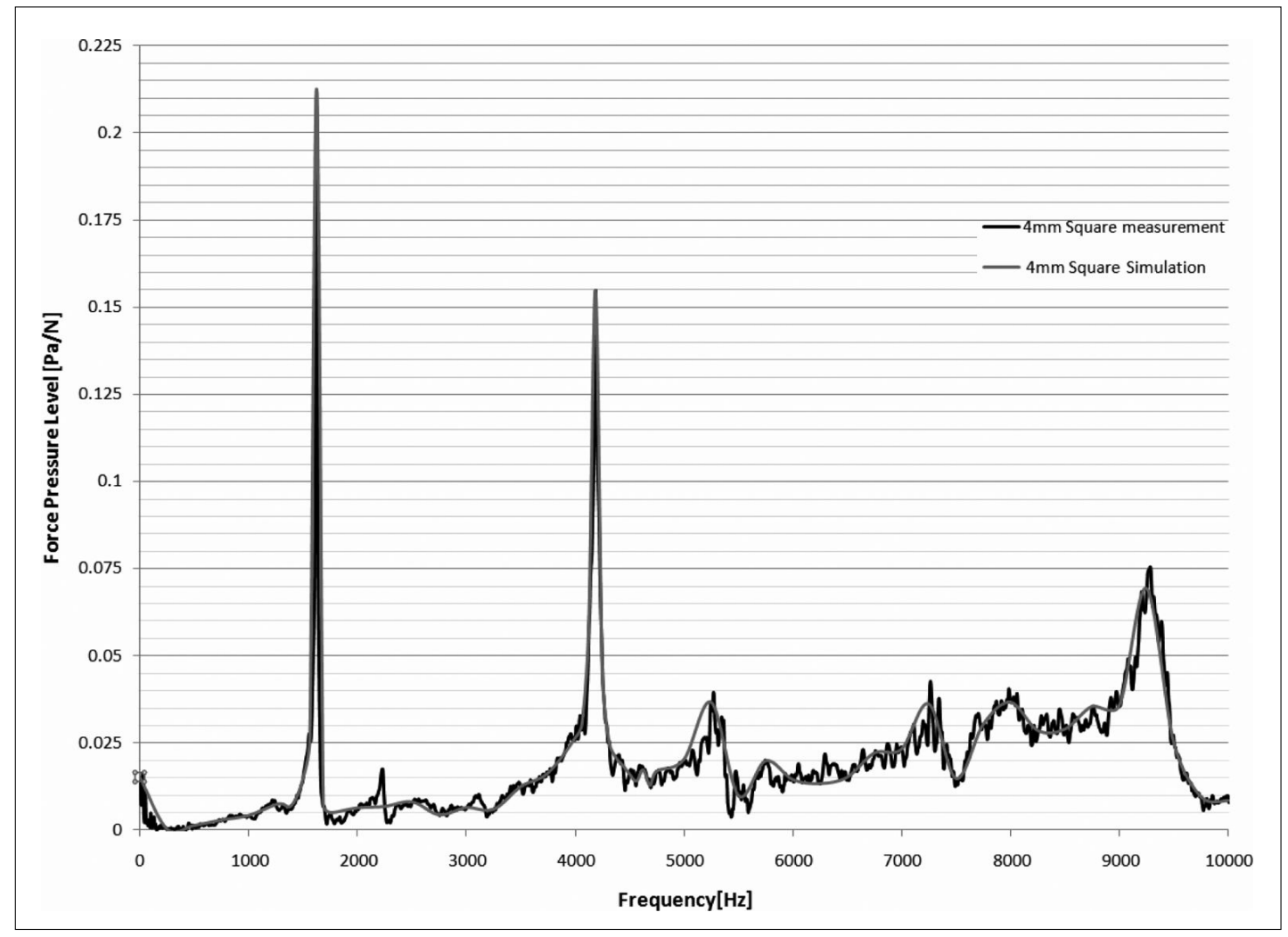

Fig. 73. $4 \mathrm{~mm}$ square EIDL - measurement Vs. simulation results. 
combined EIDL configuration is by far the most efficient case among the examined configurations.

\subsection{Finite element simulation validation}

In order to validate the integrity of the previous FE models, a comparison between the measurement data and the simulation results is given in Fig. 73. The complexity of the detailed Finite element model coupled with the vast amount of degree of freedoms and the wide band of frequency range, demanded a high performance computer server (HPC) working for several days. Even with this state of art simulation software (MSC/Nastran) and HPC, the simulation was limited to a $10 \mathrm{kHz}$ frequency range (due to a limit of 1000 modes of calculation).

Having the limitations in mind, only the $4 \mathrm{~mm}$ square configuration was simulated. Figure 73 shows a fair correlation between the simulation results and the measured data. The simulation result matches the measurements in the lower frequency band better then the upper frequency band. That is predicted due to the limited capacity of the FE model to capture the high order of modes of the model dynamic behaviour.

\section{Summary}

In order to achieve a profound method with wider bandwidth of vibration attenuation, the multi modal dynamic absorber (MMDA) is introduced. The MMDA provides a new vision of a unique mechanical structure that has numerous resonances over a wide frequency range and therefore, provides wideband vibration attenuation. The MMDA structure is based on an expansion upon the concept of the classical tuned dynamic absorber (TDA) theory. The MMDA's advantage of having numerous resonances over a wide frequency range served as a powerful dynamic absorber and results in impressive wide frequency range vibration attenuator. A number of MMDA configurations were simulated and proven experimentally to have above potential.

A direct continuation from the development of the multi modal dynamic absorber was expanding it to the conceptual model of the EIDL. This is done in order to achieve a conceptual model that has a global, wide frequency range and effective dynamic absorption. Thanks to the simple design of the EIDL, it allows a simple attachment to any optical system. A wide simulation work has been made to investigate the EIDL vibration attenuation capacity. An extensive experimental work had successfully validated the effectiveness using an embedded EIDL to reduce noise radiation. The tested mechanical system used was an adequate replica of the imager's optical bench and serves as a good example for the simplicity of incorporating the EIDL. It has shown that adding EIDL had contributed to noise export reduction of the system at a wide frequency band. The noise levels were reduced to a staggering $1 \%$ of the reference (without EIDL) noise levels, over an impressive $20 \mathrm{kHz}$ frequency range. This is achieved through a minimum system design alteration and a negligible weight addition.

As shown in Section 4.3, the EIDL configuration that revealed the most effective noise suppression capacity was the 'combined' sample. This configuration of EIDL inhabits three different ball diameters arranged in an unusual order as shown in Fig. 60 An interesting future work from this research would be investigating why does a disordered EIDL has better absorption capacity than an ordered one (shown in Fig. 72). Moreover, it would be beneficial to investigate how the level of disorder influences the EIDL absorption capacity.

\section{References}

[1] B.G. Korenev and L.M. Reznikov, Dynamic vibration absorber. Theory and technical applications, John Wiley and Sons, 1993.

[2] V.I. Babitsky and A.M. Veprik, Damping of beam forced vibration by a moving washer, Journal of Sound and Vibration 166(1) (1993), 77-85.

[3] H.K. Gary and B.F. John, Designing quiet structures: a sound power minimization approach, Academic Press, London, 1997.

[4] M.J. Brennan and J. Dayou, Global Control of Vibration Using a Tunable Vibration Neutralizer, Journal of Sound and Vibration 232(3) (2000), 585-600.

[5] E. Eşref and Ö Levent, Vibration Absorbers as Controllers, Istanbul, Turkey, Boğaziçi University, Istanbul, Turkey, 1994.

[6] A. Akay, Z. Xu, A. Carcaterra and I.M. Koc, Experiments on vibration absorption using energy sinks, The Journal of the Acoustical Society of America 118(5) (2005), 3043.

[7] I.M. Koc, A. Akay, A. Carcaterra and Z. Xu, Energy sinks: Vibration absorption by an optimal set of undamped oscillators, Journal of the Acoustical Society of America 118(5) (2005), 3031-3042. 
[8] Arpaci, Annular Plate Dampers Attached To Continuous Systems, Journal of Sound and Vibration 191(5) (1996), 781.

[9] M.D. Rao, Recent applications of viscoelastic damping for noise control in automobiles and commercial airplanes, Journal of Sound and Vibration 262 (2003), 457-474.

[10] P. Marcotte, C.R. Fuller and P. Cambou, Control of the noise radiated by a plate using a distributed active vibration absorber (DAVA), Shock and Vibration Digest 32(5) (2000).

[11] Kamal Idrisi, Marty E. Johnson, Daniel Theurich and James P. Carneal, A study on the characteristic behaviour of mass inclusions added to a poro-elastic layer, Journal of Sound and Vibration (2010), doi:10.1016/j.jsv.2010.04.001. 

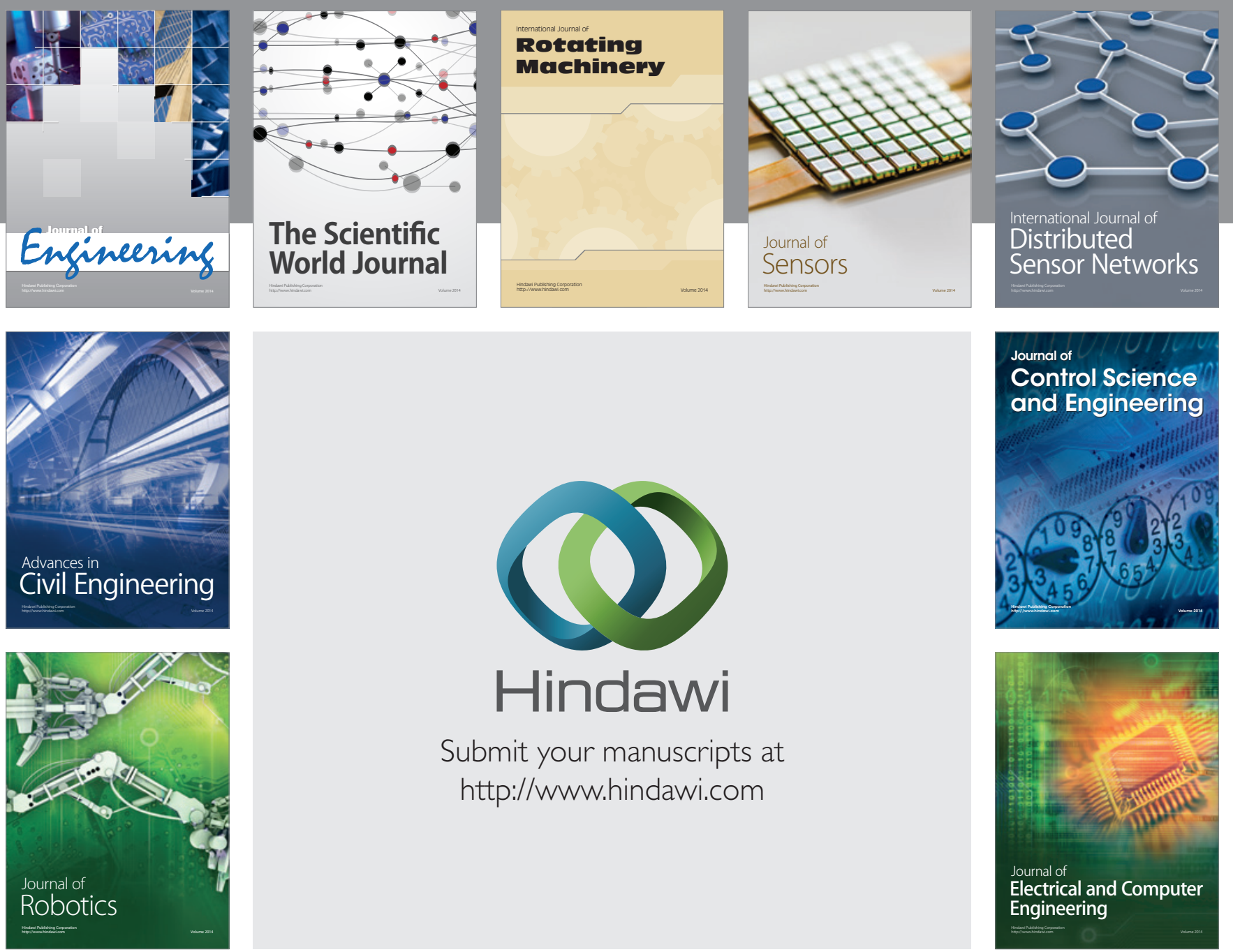

Submit your manuscripts at

http://www.hindawi.com
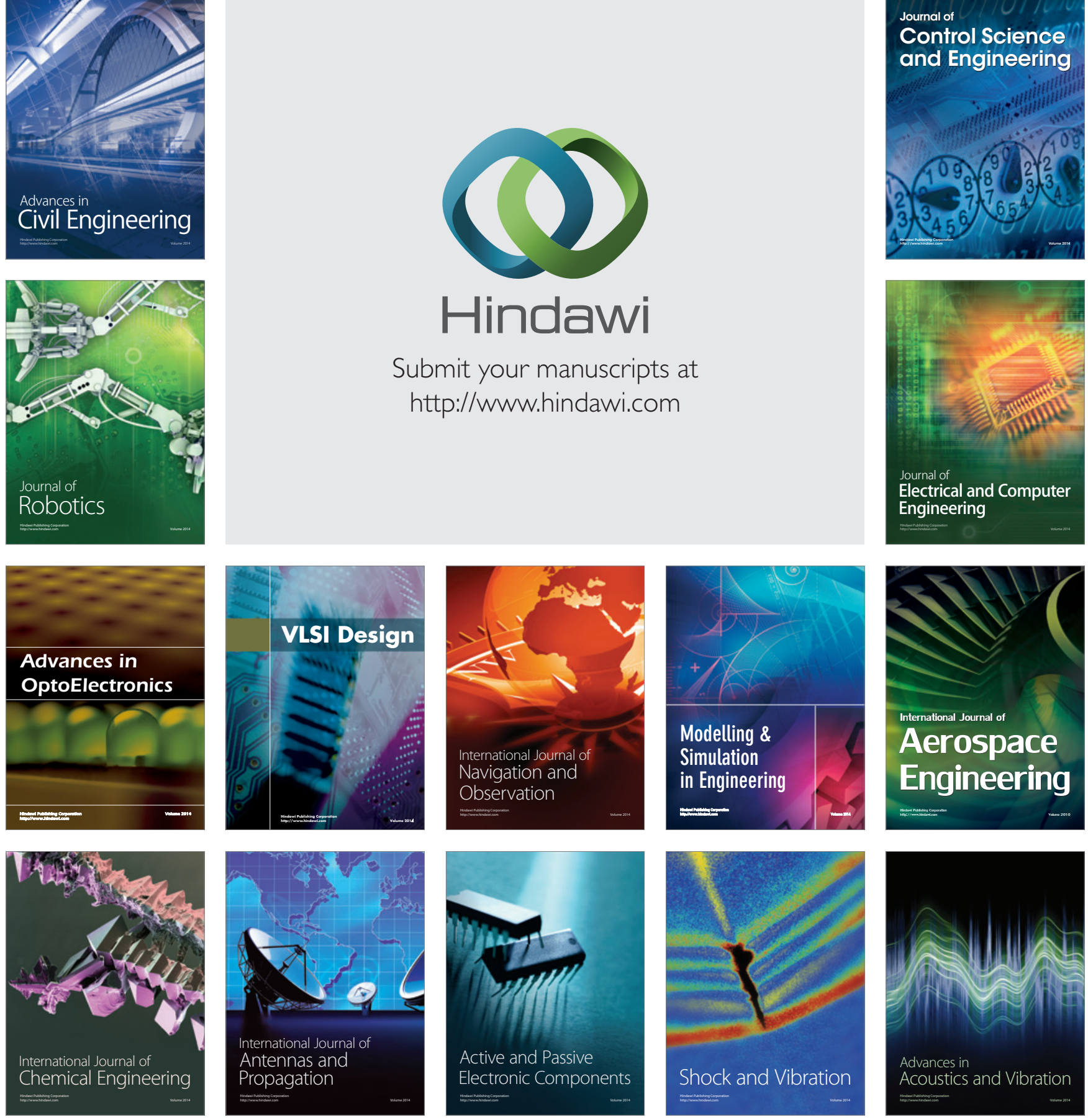